\title{
X-ray monitoring of classical novae in the central region of $\mathbf{M} 31$
}

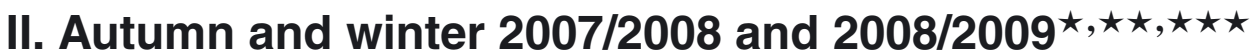

\author{
M. Henze ${ }^{1}$, W. Pietsch ${ }^{1}$, F. Haberl ${ }^{1}$, M. Hernanz ${ }^{2}$, G. Sala ${ }^{3}$, D. Hatzidimitriou ${ }^{4,5}$, \\ M. Della Valle $6,7,8$, A. Rau ${ }^{1}$, D. H. Hartmann ${ }^{9}$, and V. Burwitz ${ }^{1}$
}

\author{
1 Max-Planck-Institut für extraterrestrische Physik, Giessenbachstraße, 85748 Garching, Germany \\ e-mail: mhenze@mpe.mpg.de \\ 2 Institut de Ciències de l'Espai (CSIC-IEEC), Campus UAB, Fac. Ciències, 08193 Bellaterra, Spain \\ 3 Departament de Física i Enginyeria Nuclear, EUETIB (UPC-IEEC), Comte d'Urgell 187, 08036 Barcelona, Spain \\ 4 Department of Astrophysics, Astronomy and Mechanics, Faculty of Physics, University of Athens, Panepistimiopolis, \\ 15784 Zografos, Athens, Greece \\ 5 Foundation for Research and Technology Hellas, IESL, Greece \\ 6 European Southern Observatory (ESO), 85748 Garching, Germany \\ 7 INAF-Napoli, Osservatorio Astronomico di Capodimonte, Salita Moiariello 16, 80131 Napoli, Italy \\ 8 International Centre for Relativistic Astrophysics, Piazzale della Repubblica 2, 65122 Pescara, Italy \\ 9 Department of Physics and Astronomy, Clemson University, Clemson, SC 29634-0978, USA
}

Received 7 October 2010 / Accepted 6 July 2011

\begin{abstract}
Context. Classical novae (CNe) represent the major class of supersoft X-ray sources (SSSs) in the central region of the galaxy M 31. Aims. We performed a dedicated monitoring of the M 31 central region with XMM-Newton and Chandra between Nov. 2007 and Feb. 2008 and between Nov. 2008 and Feb. 2009, respectively, to find SSS counterparts of CNe, determine the duration of their SSS phase, and derive physical outburst parameters.

Methods. We systematically searched our data for X-ray counterparts of CNe, determined their X-ray light curves and characterised their spectra using blackbody fits and white dwarf (WD) atmosphere models. Additionally, we determined luminosity upper limits for all previously known X-ray emitting novae that are not detected any more and for all CNe in our field of view with optical outbursts between one year before the start of the X-ray monitoring (Oct. 2006) and its end (Feb. 2009).

Results. We detected $17 \mathrm{X}$-ray counterparts of $\mathrm{CNe}$ in M 31, only four of which were previously known. These latter sources are still active 12.5, 11.0, 7.4 and 4.8 years after the optical outburst. In addition, we detected three known SSSs without a nova counterpart. Four novae displayed short SSS phases $(<100 \mathrm{~d})$. Based on these results and previous studies we compiled a catalogue of all novae with SSS counterparts in M 31 known so far. We used this catalogue to derive correlations between the following X-ray and optical nova parameters: turn-on time, turn-off time, blackbody temperature (X-ray), $t_{2}$ decay time and expansion velocity of the ejected envelope (optical). Temperatures derived from blackbody fits and WD atmosphere models were found to characterise the effective SSS temperatures almost equally well. Furthermore, we found a first hint for the existence of a difference between SSS parameters of novae associated with the stellar populations of the M 31 bulge and disk. Additionally, we conducted a Monte Carlo Markov chain simulation on the intrinsic fraction of novae with SSS phase. This simulation showed that the high fraction of novae without detected SSS emission might be explained by the inevitably incomplete coverage with X-ray observations in combination with a large part of novae with short SSS states, as expected from the WD mass distribution.

Conclusions. Our results confirm that novae are the major class of SSSs in the central region of M 31. The catalogue of novae with $\mathrm{X}$-ray counterpart, mainly based on our X-ray monitoring, contains valuable insight into the physics of the nova process. In order to verify our results with an increased sample, more monitoring observations are needed.
\end{abstract}

Key words. galaxies: individual: M 31 - novae, cataclysmic variables - X-rays: binaries - stars: general

\section{Introduction}

This is the second of two papers analysing data from recent $\mathrm{X}$-ray monitoring campaigns for classical novae in the central region of our neighbour galaxy $\mathrm{M} 31$. In the first paper (Henze et al. 2010, hereafter Paper I) we presented the results of an

* Partly based on observations with XMM-Newton, an ESA Science Mission with instruments and contributions directly funded by ESA Member States and NASA.

$\star \star$ All tables are available in electronic form at http://www . aanda.org

$\star \star \star$ Table 9 is also available at the CDS via anonymous ftp to cdsarc.u-strasbg.fr (130.79.128.5) or via

http://cdsarc.u-strasbg.fr/viz-bin/qcat?]/A+A/533/A52 earlier campaign from June 2006 to March 2007. Here we report our findings from the second and third monitoring seasons from November 2007 until February 2008 and from November 2008 until February 2009, respectively, and compile and analyse a catalogue of all novae with X-ray emission in M 31.

We briefly recall the physical processes involved in the nova phenomenon and define the terms used in this work. For a more detailed introduction see Paper I.

Classical novae $(\mathrm{CNe})$ originate from thermonuclear explosions on the surface of white dwarfs (WDs) in cataclysmic binaries. Hydrogen-rich matter is transferred from the companion star and accumulates on the surface of the WD until hydrogen ignition starts a thermonuclear runaway in the degenerate matter of the WD envelope. The resulting expansion of the hot envelope 
causes the brightness of the WD to rise by $\sim 12$ mag within a few days. Mass is ejected at high velocities (see Warner 1995; Hernanz 2005, and references therein).

A fraction of the H-burning envelope can remain in steady burning on the surface of the WD (Starrfield et al. 1974; Sala \& Hernanz 2005), powering a supersoft X-ray source (SSS) that can be observed directly once the ejected envelope becomes sufficiently transparent (Starrfield 1989; Krautter 2002). The turnon time of the SSS is defined here as the time when the SSS becomes visible to us owing to the decreasing opacity of the ejected material. This time scale is determined by the fraction of mass ejected in the outburst. The ceasing of H-burning in the WD envelope causes the disappearance of the SSS and is defined as turn-off time. The duration of the SSS state is inversely related to the mass of the WD for a given hydrogen-mass fraction in the remaining envelope (José \& Hernanz 1998; Yaron et al. 2005). On the other hand, the larger the hydrogen content, the longer the duration of the SSS state for a given WD mass (see Tuchman \& Truran 1998; Sala \& Hernanz 2005; Hachisu \& Kato 2006).

$\mathrm{X}$-ray light curves therefore can provide important clues on whether a WD accumulates matter over time to become a potential progenitor for a type Ia supernova (SN-Ia). The duration of the SSS state provides the only direct indicator of the postoutburst hydrogen envelope mass in CNe. For massive WDs, the expected SSS duration is very short $(<100 \mathrm{~d})$ (Tuchman \& Truran 1998; Sala \& Hernanz 2005).

Owing to its proximity to the Galaxy (distance $780 \mathrm{kpc}$; Holland 1998; Stanek \& Garnavich 1998) and its moderate Galactic foreground absorption $\left(N_{\mathrm{H}} \sim 6.7 \times 10^{20} \mathrm{~cm}^{-2}\right.$, Stark et al. 1992), M 31 is a unique target for $\mathrm{CN}$ surveys and has played a key role in nova population studies (e.g. Ciardullo et al. 1987; Capaccioli et al. 1989; Shafter \& Irby 2001). The idea of two distinct optical nova populations was first introduced by Duerbeck (1990) and Della Valle et al. (1992) based on data on Galactic novae. They suggested that fast novae (time of decline by 2 mag from maximum magnitude $t_{2} \leq 12$ days) are mainly associated with the disk of the Galaxy or are concentrated close to the Galactic plane, whereas slower novae are mostly present in the bulge region of the Galaxy or at greater distances from the Galactic plane. Another argument in favour of this idea came from Della Valle \& Livio (1998), who reported systematic spectroscopic differences in the optical between Galactic bulge and disk novae. They found that novae that can be classified as "Fe II" novae in the system of Williams (1992) tend to be associated with the bulge of the Galaxy, whereas "He/N" novae mostly belong to the disk. According to Williams (1992), novae with prominent Fe II lines in the optical spectrum evolve more slowly with lower expansion velocities and lower levels of ionisation. On the other hand, novae with stronger lines of He and $\mathrm{N}$ have high expansion velocities and a rapid spectral evolution. These novae are often observed to also display strong Ne lines, which may point to a relatively massive ONe WD in the binary system (see e.g. Shafter \& Quimby 2007). ONe WDs are believed to be initially more massive than CO WDs (José \& Hernanz 1998) and therefore to have more massive progenitors. Therefore, a correlation of these objects with the younger (disk) stellar population seems plausible. There is an on-going controversy about which of the two populations dominates the nova rate in M 31 and the Galaxy (for an early overview see Hatano et al. 1997). In this paper, we study for the first time the differences between M 31 bulge and disk novae in the X-ray regime.

The observed X-ray spectra of $\mathrm{CNe}$ are characterised in this work using blackbody models. We are aware that blackbody fits to SSS spectra are an oversimplification of the complex nova physics and therefore do not provide a physically accurate model (see e.g. Greiner et al. 1991; Kahabka \& van den Heuvel 1997, and references therein). Ideally, one would like to apply realistic stellar atmosphere models, which have been successfully tested on X-ray grating spectra of Galactic $\mathrm{CNe}$ and include, among other things, non-local thermodynamic equilibrium (NLTE) and an expanding envelope (see e.g. Petz et al. 2005; Nelson et al. 2008b; van Rossum \& Ness 2010). However, these models require a large set of free parameters (such as envelope abundances, expansion velocities) to be fitted to provide a realistic description of the nova atmosphere (van Rossum \& Ness 2010). Unfortunately, novae in M 31 are generally not bright enough to ensure sufficient photon statistics for realistic atmosphere models. Furthermore, even for brighter novae there is the problem that our field is too crowded to derive grating spectra of individual objects (see Fig. 1), consequently limiting spectral analysis to low-resolution (XMM-Newton EPIC PN) CCD detectors, which are not sufficient to resolve the detailed structure of realistic atmosphere models (see also Ness et al. 2011).

Another reason for using blackbody fits is that in the past they have been applied to SSS spectra of M 31 CNe by Pietsch et al. (2005a, hereafter PFF2005), Pietsch et al. (2007d, hereafter PHS2007) and various other authors. This fact is important for our goal to conduct a statistical study of $\mathrm{CNe}$ in $\mathrm{M} 31$, because it ensures a homogeneous treatment of current and literature sources. We assume in this work that temperatures derived from blackbody fits provide means to characterise the actual temperature of the SSS with sufficient accuracy to study statistical trends. We validate this assumption by comparing the blackbody temperatures to temperatures derived from NLTE WD atmosphere models for the subset of nova SSS spectra analysed in this work and in Paper I. The atmosphere models used for this comparison (Rauch 2003) are applicable to M 31 SSS spectra because they have been reduced to an one-parametric model.

In Sect. 2 we describe our X-ray observations and data analysis, including the fitting of SSS spectra. Results from the observations are presented in Sect. 3 and a catalogue of all nova SSSs in M 31 is compiled in Sect. 4. An extensive discussion of this catalogue is the subject of Sect. 5. We conclude with a summary in Sect. 6.

\section{Observations and data analysis}

The X-ray data used in this work were obtained in a joint XMMNewton/Chandra programme (PI: W. Pietsch). We monitored the M 31 central region with the XMM-Newton European Photon Imaging Camera (EPIC) and the Chandra High-Resolution Camera Imaging Detector (HRC-I) during autumn and winter 2007/8 and 2008/9. All observations were pointed at the M 31 centre (RA: 00:42:44.33, Dec: +41:16:07.5; J2000). The individual observations were typically separated by ten days, in contrast to the 40-day spacing in Paper I. We changed our monitoring strategy to account for a significant percentage of $\mathrm{CNe}$ in M 31 with short SSS phases found in our earlier work (see PHS2007). The dates, observation identifications (ObsIDs), and dead-time corrected exposure times of the individual observations are given in Table 1. For the rest of the paper, 2007/8 and 2008/9 will indicate the corresponding monitoring campaign.

In the XMM-Newton observations, the EPIC PN and MOS instruments were operated in the full-frame mode. We used the thin filter for PN and the medium filter for MOS.

Our data reduction and analysis techniques differ from the standard processing for both XMM-Newton EPIC and Chandra HRC-I and were described in detail in Paper I. The only change 




Fig. 1. Logarithmically scaled, three colour XMM-Newton EPIC images of the central area of M 31 combining PN, MOS1, and MOS2 data of all five observations for 2007/8 (top panel) and 2008/9 (bottom panel). Red, green, and blue show the (0.2-0.5) keV, (0.5-1.0) keV and (1.0-2.0) keV bands. Supersoft X-ray sources show up in red. The data in each colour band were binned in $2^{\prime \prime} \times 2^{\prime \prime}$ pixels and smoothed using a Gaussian of FWHM 5". The counterparts of optical novae detected in this work are marked with white circles. For M31N 1997-11a, M31N 2003-08c, M31N 2004-01b, M31N 2007-11a, M31N 2008-05b, and M31N 2008-06a only the positions are designated, because they are not visible in these images but are detected in Chandra images. The non-nova SSSs detected in this work are marked with white boxes. The large white box includes the central region of M 31, which is shown as a Chandra composite in Fig. 2.

in this work was the update of all procedures to the most recent versions of the instrument-dependent analysis software: XMMSAS (XMM-Newton Science Analysis System; Gabriel et al. 2004) ${ }^{1}$ v9.0 and CIAO (Chandra Interactive Analysis of

\footnotetext{
${ }^{1}$ http://xmm.esac.esa.int/external/xmm_data_analysis/
}

Observations; Fruscione et al. 2006) $)^{2}$ v4.2. The statistical analysis described in Sect. 5 was performed using the R software environment ${ }^{3}$. For spectral fitting we used XSPEC (Arnaud 1996)

\footnotetext{
${ }^{2}$ http://cxc.harvard.edu/ciao/

${ }^{3}$ http://www.r-project.org
} 
v12.5.0. In all our spectral models we used the TübingenBoulder ISM absorption model (TBabs in XSPEC) together with the photoelectric absorption cross-sections from BalucinskaChurch \& McCammon (1992) and ISM abundances from Wilms et al. (2000). The statistical confidence ranges of parameters derived from spectral fits (e.g. temperature, $N_{\mathrm{H}}$ ) are $90 \%$ unless stated otherwise.

We parameterise the temperature of the SSS spectra derived in this work using best-fit blackbody temperatures. To check if the blackbody temperatures describe the actual source temperature with sufficient accuracy to detect statistical trends, we additionally fitted all spectra discussed here and in Paper I with NLTE WD atmosphere models. For this purpose, we made use of publicly available ${ }^{4}$ pre-calculated grids for the spectral energy distribution of hot compact stars computed by Rauch (2003). These grids use the effective temperature of the star as the only parameter and are based on plane-parallel NLTE models in hydrostatic and radiative equilibrium (see also Rauch et al. 2010). The models were computed using the Tübingen model-atmosphere package (TMAP, Rauch et al. 2010; Rauch \& Deetjen 2003).

We decided to use a model grid $^{5}$ that contains all elements from H to Ca (Rauch 1997), because it allowed us to cover a wide range of effective temperatures (although with restriction to models with $\log g=9.0$; see also Henze et al. 2009a). This grid includes two different atmosphere abundances, Galactic halo or solar, both of which were tested in this work.

This approach clearly has limitations. The assumptions of plane-parallel and hydrostatic equilibrium are certainly not valid for a rapidly evolving nova atmosphere. Moreover, the elemental abundances of the model would need to be adjusted individually for every nova, as would the gravitational acceleration of the WD.

Note that all luminosities given in this paper were not computed from spectral fits but assume a generic $50 \mathrm{eV}$ blackbody spectrum with Galactic foreground absorption $\left(N_{\mathrm{H}} \sim\right.$ $6.7 \times 10^{20} \mathrm{~cm}^{-2}$ ). These "equivalent luminosities" (or " $L_{50}$ ") are used to compare our results to the earlier work of PHS2007 and to Paper I, where luminosities were determined similarly. Consequently, we only describe the relative brightness evolution of individual sources (see Sect. 3.1).

Additionally, during the time of both campaigns, there were twelve observations including the M 31 central region with Chandra ACIS-I and 62 observations with the Swift X-ray Telescope (XRT). We checked all these observations for additional information on the novae found in our monitoring. Whereas in the ACIS-I data none of the novae was detected, in the Swift XRT observations three novae were visible. The information contained in these data has already been published by Henze et al. (2009a,b) and Bode et al. (2009) for novae M31N 2007-06b, M31N 2007-11a, and M31N 2007-12b, respectively. Furthermore, there are no non-detections of SSS counterparts, which would result in additional constraints on their turn-on or turn-off time. The main reasons for this are (a) the temporal distribution of the observations and (b) the smaller field of view of the Swift XRT together with the fact that many observations were not pointed directly at the M 31 centre and therefore do not cover all nova positions. Therefore, the Chandra ACIS-I and Swift XRT data do not yield additional information

\footnotetext{
4 http://astro.uni-tuebingen.de/ rauch/

5 http://astro. uni-tuebingen.de/ rauch/TMAF/flux_ H-Ca.html
}

on the novae discovered in this paper and will not be discussed further.

We also conducted a general search for SSSs in our XMMNewton data, following the approach adopted by PFF2005 using hardness ratios computed from count rates in energy bands 1 to $3(0.2-0.5 \mathrm{keV}, 0.5-1.0 \mathrm{keV}, 1.0-2.0 \mathrm{keV})$ to classify a source. These authors defined hardness ratios and errors as

$H R_{i}=\frac{B_{i+1}-B_{i}}{B_{i+1}+B_{i}}$ and $E H R_{i}=2 \frac{\sqrt{\left(B_{i+1} E B_{i}\right)^{2}+\left(B_{i} E B_{i+1}\right)^{2}}}{\left(B_{i+1}+B_{i}\right)^{2}}$,

for $i=1,2$, where $B_{i}$ and $E B_{i}$ denote count rates and corresponding errors in band $i$ as derived by emldetect. Pietsch et al. (2005a) classified sources as SSSs if they fulfil the conditions $H R_{1}<0.0$ and $H R_{2}-E H R_{2}<-0.4$. In this work we use the same criteria.

\section{Results}

In the two monitoring campaigns 2007/8 and 2008/9 we detected 17 X-ray counterparts of CNe in M 31 in total. In addition, three SSSs without a nova counterpart were found. The positions of all objects are indicated in Figs. 1 and 2, which show merged images from all observations in 2007/8 and 2008/9 for XMM-Newton and Chandra, respectively. X-ray measurements of all optical nova counterparts that were detected at least with a $2 \sigma$ significance (for XMM-Newton in the $0.2-1.0 \mathrm{keV}$ band, combining all EPIC instruments) are given in Tables 2 and 4. Tables 5-7 present $3 \sigma$ upper limits for optical novae undetected in X-rays, which were found as X-ray sources in Paper I or which had their outbursts within a year before or during the monitoring campaigns. For XMM-Newton, these upper limits where determined from the more sensitive EPIC PN camera if possible (see also PFF2005).

Tables 2-7 contain the following information: the name, coordinates, and outburst date of the optical nova (taken from the online catalogue of PHS2007 ${ }^{6}$ ), the distance between optical and X-ray source (if detected), the X-ray observation and its time lag with respect to the optical outburst, the unabsorbed equivalent $\mathrm{X}$-ray luminosity or its upper limit in the $0.2-1.0 \mathrm{keV}$ band assuming a $50 \mathrm{eV}$ blackbody spectrum with Galactic foreground absorption, and comments. Model parameters from WD atmosphere fits are compared to blackbody parameters in Table 3.

\subsection{X-ray counterparts of optical novae in M 31 known previously}

Four of the 17 detected X-ray counterparts (see Table 2) were already seen in observations presented in Paper I: M31N 199608b, M31N 1997-11a, M31N 2001-10a and M31N 2004-05b. All of them had been previously detected in PHS2007 and were still visible at the end of the 2008/9 campaign.

Nova M31N 1996-08b remained active for 12.5 years after the optical discovery. Its X-ray spectrum did not change in 2007/8 and 2008/9 with respect to Paper I. Merging the new data with the spectra obtained in Paper I we derived a new best-fit blackbody temperature $k T=21_{-13}^{+8} \mathrm{eV}$ and an absorption $N_{\mathrm{H}}=\left(1.4_{-0.8}^{+1.2}\right) \times 10^{21} \mathrm{~cm}^{-2}$. The values of both parameters are almost the same as in Paper I but the errors are significantly reduced. The equivalent luminosity of the source, given in Table 2, did not change significantly with respect to the 2006/7 observations reported in Paper I. Similarly, the X-ray light curve over 2007/8 and 2008/9 seems stable. Discrepancies between

\footnotetext{
${ }^{6}$ http://www . mpe.mpg.de/ m31novae/opt/m31/index.php
} 
M. Henze et al.: X-ray monitoring of classical novae in M 31 in 2007/8 and 2008/9. II.

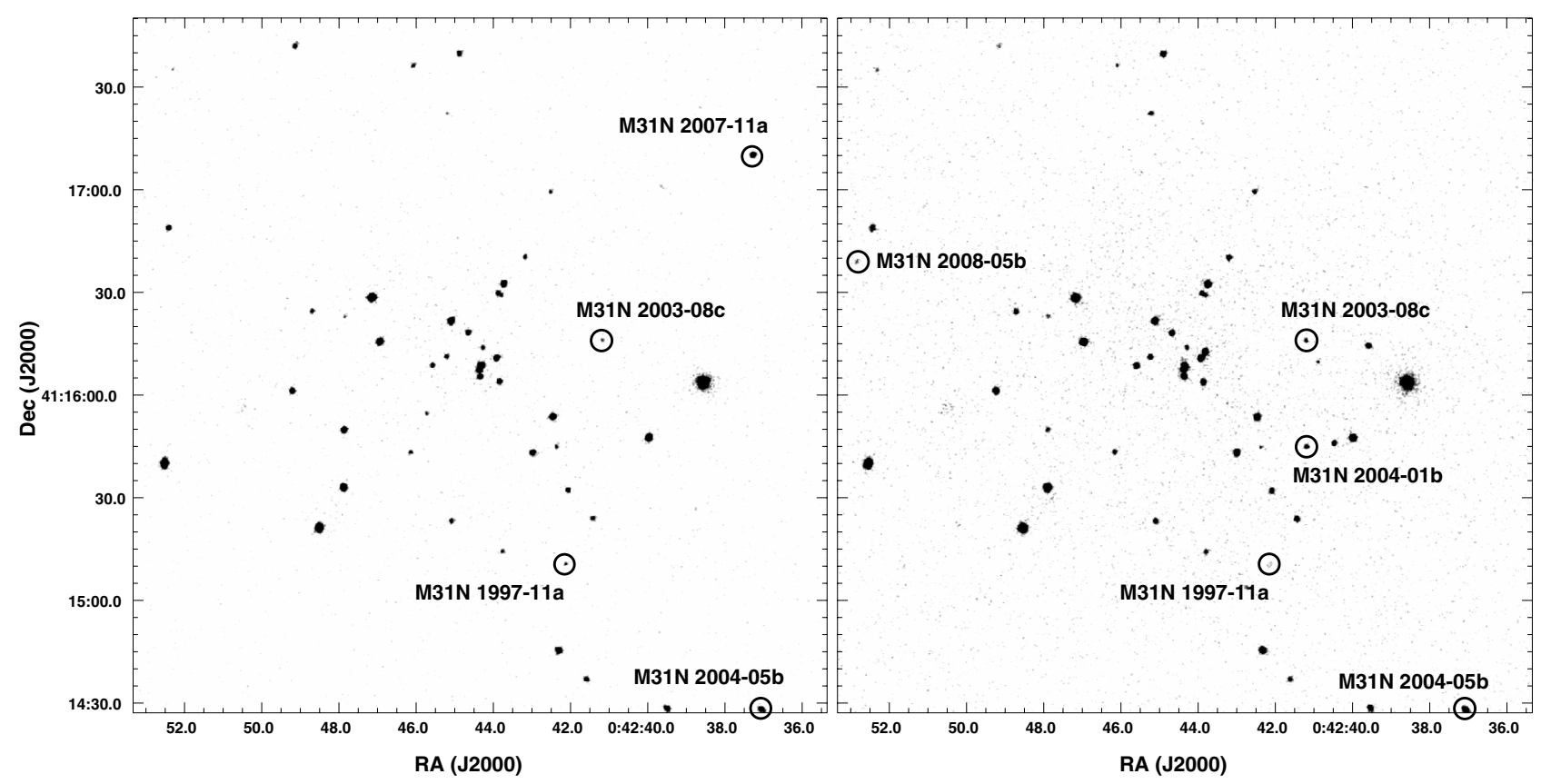

Fig. 2. Logarithmically scaled Chandra HRC-I images of the innermost 3.3 $\times 3.3$ of M 31 combining all observations of 2007/8 (left panel) and 2008/9 (right panel). The images were not binned (HRC electronic pixel size $=0$ !'13) but were smoothed with a Gaussian of FWHM 0".5. The $\mathrm{X}$-ray counterparts of novae in the field are marked with black circles.

XMM-Newton and Chandra luminosities are likely to arise because of the differences of the generic spectral model used to compute the equivalent luminosities in Table 2 and the actual source spectrum. See Paper I for a discussion of this issue.

Nova M31N 1997-11a is situated close to the centre of M 31. The X-ray light curve of the source shows a decline from an average $L_{50}=(7.6 \pm 0.8) \times 10^{36} \mathrm{erg} \mathrm{s}^{-1}$ in $2006 / 7$ (Paper I) through $L_{50}=(3.2 \pm 0.4) \times 10^{36} \mathrm{erg} \mathrm{s}^{-1}$ in $2007 / 8$ to $L_{50}=$ $(0.6 \pm 0.2) \times 10^{36} \mathrm{erg} \mathrm{s}^{-1}$ in $2008 / 9$ (see Table 2). However, note the increase of equivalent luminosity by a factor of two during observation 8529 (3689 days after outburst), which is interrupting the overall decline. In the last campaign, 11.0 years after the optical outburst, the source is so faint that it is only detected in the merged Chandra data.

Nova M31N 2001-10a is still active 7.4 years after the optical discovery. The best-fit parameters for modelling the combined XMM-Newton 2007/8 and 2008/9 spectra are $k T=14_{-8}^{+5} \mathrm{eV}$ and $N_{\mathrm{H}}=(2.2 \pm 0.5) \times 10^{21} \mathrm{~cm}^{-2}$. These values are not significantly different from the results presented in Paper I. Fitting the spectra derived here together with the data from Paper I resulted in a slightly better constrained blackbody temperature $k T=14_{-7}^{+4} \mathrm{eV}$. The X-ray equivalent luminosity averaged over the individual monitoring campaigns was constant within the errors at $L_{50} \sim 2.5 \times 10^{36} \mathrm{erg} \mathrm{s}^{-1}$ (XMM-Newton data). The X-ray light curve during the $2007 / 8$ and 2008/9 campaigns did not vary significantly either (see Table 2). Differences between XMMNewton and Chandra equivalent luminosities are again owing to the generic spectrum that we used for converting count rates to fluxes and that does not take into account the low source temperature.

Nova M31N 2004-05b is still detected 4.8 years after the optical outburst. This source seems to undergo a spectral evolution, indicated by significantly different blackbody temperature and absorption for the two campaigns: $2007 / 8: k T=(31 \pm 4) \mathrm{eV}$, $N_{\mathrm{H}}=\left(1.4_{-0.2}^{+0.3}\right) \times 10^{21} \mathrm{~cm}^{-2} ; 2008 / 9: k T=(42 \pm 6) \mathrm{eV}, N_{\mathrm{H}}=$ $\left(0.8_{-0.2}^{+0.3}\right) \times 10^{21} \mathrm{~cm}^{-2}$. The $2007 / 8$ parameters are compatible with the best-fit values obtained in Paper I. The X-ray light curve of M31N 2004-05b during 2007/8 (see Table 2) was relatively stable with similar equivalent luminosity as measured in 2006/7 (see Paper I). The 2008/9 blackbody luminosities might be slightly lower than those in $2007 / 8$, but the effect is not significant and might be caused by the spectral changes that the source is experiencing. Additionally, there was a strong increase in equivalent luminosity by a factor of about two in observation 8527 (1279 days after outburst) with respect to previous and subsequent observations (see Table 2).

\subsection{X-ray counterparts of optical novae in M 31 discovered in this work}

In total, 13 sources were detected for the first time in the observations of the campaigns presented here (see Table 4). The following three new sources exhibit extraordinary properties and were already discussed in detail in previous work: M31N 2007-06b was the first nova in a M 31 globular cluster (Henze et al. 2009a). M31N 2007-11a had a well documented, very short SSS phase (Henze et al. 2009b). M31N 2007-12b was discussed for its Xray light curve variability in Pietsch et al. (2011) and Orio et al. (2010). The possibility that it is a recurrent nova was examined in Bode et al. (2009).

\subsubsection{M31N 2003-08c}

The optical nova was discovered by Fiaschi et al. (2003) on 2003-10-16 and spectroscopically confirmed by di Mille et al. (2003). A faint $\left(L_{50} \sim 3.5 \times 10^{36} \mathrm{erg} \mathrm{s}^{-1}\right)$ X-ray counterpart first showed up in the Chandra observations of 2007/8 (about 1540 days after the optical outburst, see Table 4). Owing to the position of the source close to the M 31 centre, our XMMNewton data suffer from source confusion and can only provide luminosity upper limits that are higher than the measured equivalent luminosities inferred from Chandra. For Table 9 we adopt as turn-on time of the source the midpoint between the last observation from Paper I and the first Chandra observation in 2007/8. The light curve of the nova counterpart was variable by at least 
a factor of two over the course of 2007/8 and 2008/9 campaigns. The source was still detected in the last observation of $2008 / 9$, 2010 days after the outburst.

\subsubsection{M31N 2004-01b}

The optical nova candidate was discovered in the WeCAPP survey (Riffeser et al. 2001) on 2004-01-01 (see also PHS2007). An X-ray counterpart with an average equivalent luminosity $L_{50}=(6.1 \pm 0.5) \times 10^{36} \mathrm{erg} \mathrm{s}^{-1}$ was found in the Chandra data of 2008/9 (1773 days after outburst). This object was not detected in the Chandra data of the 2007/8 campaign with an upper limit of $L_{50}<1.6 \times 10^{36} \mathrm{erg} \mathrm{s}^{-1}$ in observation 8530 (1446 days after outburst, see Table 4). The turn-on time of the source given in Table 9 was assumed to be the midpoint between the observations 8530 and 9825 . Because the source is located close to the M 31 centre, source confusion prevented XMM-Newton from detecting it. Towards the end of the 2008/9 campaign the equivalent luminosity of the source significantly increased up to $L_{50}=(11.1 \pm 1.6) \times 10^{36} \mathrm{erg} \mathrm{s}^{-1}$ in the second last Chandra observation 1873 days after the optical outburst.

\subsubsection{M31N 2006-06b}

The optical nova candidate was discovered independently by Ries \& Riffeser (2006) and Hornoch ${ }^{7}$ on 2006-06-06. Both authors report evidence pointing towards a slowly rising nova. An X-ray counterpart was detected in the first 2008/9 observation, 909 days after the optical outburst. Nothing was found at this position in the 2007/8 campaign, with an upper limit of $L_{50}<1.6 \times 10^{36} \mathrm{erg} \mathrm{s}^{-1}$ in XMM-Newton observation 0505720601 (634 days after outburst, see Table 4). The source was visible in XMM-Newton and Chandra data until the end of the 2008/9 campaign with an average equivalent luminosity $L_{50}=(3.6 \pm 0.3) \times 10^{36} \mathrm{erg} \mathrm{s}^{-1}$ in Chandra data. This luminosity was increasing significantly during the time span of the monitoring. The turn-on time given in Table 9 is estimated as the midpoint between the observations 0505720601 and 9825 .

We fitted the XMM-Newton EPIC PN spectrum of the source with an absorbed blackbody model with best-fit parameters $k T=$ $37_{-15}^{+17} \mathrm{eV}$ and $N_{\mathrm{H}}=\left(0.5_{-0.2}^{+0.9}\right) \times 10^{21} \mathrm{~cm}^{-2}$. This source clearly can be classified as a SSS.

\subsubsection{M31N 2006-09c}

The optical nova was discovered independently by Itagaki ${ }^{8}$ and Quimby (2006) on 2006-09-18. Shafter et al. (2006) classified it as a Fe II nova. A faint $\left(L_{50} \lesssim 4.0 \times 10^{36} \mathrm{erg} \mathrm{s}^{-1}\right)$ X-ray counterpart was detected in the second Chandra observation of 2007/8 (426 days after outburst, see Table 4). However, the preceding Chandra observation only provided an upper limit that was higher than the detection luminosity. Therefore, we could not determine if the source was already active on a similar level during this observation. From the upper limits given in Paper I we deduce that the source was not detectable 140 days after optical outburst with an upper limit of $L_{50}<0.9 \times 10^{36} \mathrm{erg} \mathrm{s}^{-1}$. The turn-on time given in Table 9 is estimated as the midpoint between days 140 and 415 (first Chandra observation of the 2007/8 campaign). Similarly, owing to the faintness of the source it was not clear if the non-detections in the last two XMMNewton observations of $2007 / 8$ correspond to the X-ray turn-off

\footnotetext{
7 See http://www.cfa.harvard.edu/iau/CBAT_M31.html\# $2006-06 b$

8 See http://wWw.cfa.harvard.edu/iau/CBAT_M31.html\# $2006-09 c$
}

of the source. For Table 9 we therefore estimated the actual turnoff of the source to have occurred in between the last 2007/8 and the first 2008/9 observation (507 and 834 days after outburst) of XMM-Newton.

The combined XMM-Newton EPIC PN spectra of the X-ray counterpart can be fitted with an absorbed blackbody model with $k T=74_{-24}^{+20} \mathrm{eV}$ and $N_{\mathrm{H}}=\left(0.2_{-0.2}^{+0.8}\right) \times 10^{21} \mathrm{~cm}^{-2}$, which classifies this source as a SSS.

\subsubsection{M31N 2007-02b}

The optical nova was discovered by Hornoch ${ }^{9}$ on 2007-02-03. It was spectroscopically confirmed by Pietsch et al. (2007a) and Shafter ${ }^{10}$ who classified it as hybrid nova and Fe II nova respectively. An X-ray counterpart was detected in the third XMMNewton observation of 2007/8 (349 days after the optical outburst, see Table 4). However, the source is right on the edge of the XMM-Newton field of view in this observation and its position is not covered in the first two 2007/8 observations because of the changing roll angle. The large distance of the nova from the M 31 centre might also be the reason for its non-detection by Chandra in 2007/8 and 2008/9. This is because the Chandra PSF strongly degrades towards high off-axis angles, an effect that significantly decreases the detection sensitivity. We therefore assume that the source was active from the third 2007/8 until at least the last 2008/9 observation of XMM-Newton (732 days after outburst). Its equivalent luminosity significantly increased from one campaign to the next (Table 4).

The XMM-Newton EPIC PN spectra of 2007/8 and 2008/9 could be fitted with absorbed blackbody models, the parameters of which agreed within the errors. We therefore performed a simultaneous modelling of both spectra, which resulted in best fit $k T=(28 \pm 10) \mathrm{eV}$ and $N_{\mathrm{H}}=\left(2.5_{-1.1}^{+1.8}\right) \times 10^{21} \mathrm{~cm}^{-2}$, allowing us to classify this source as a SSS.

\subsubsection{M31N 2007-10b}

The optical nova was discovered by Burwitz et al. (2007) on 2007-10-13.26 UT. The start of the nova outburst was determined with the accuracy of less than a day from a nondetection on 2007-10-12.40 UT (Burwitz et al. 2007). Based on optical spectra, Rau et al. (2007) classified the object as a $\mathrm{He} / \mathrm{N}$ nova. They further reported an expansion velocity of $1450 \pm 100 \mathrm{~km} \mathrm{~s}^{-1}$ and noted that this value is atypically low for $\mathrm{He} / \mathrm{N}$ novae. An $\mathrm{X}$-ray counterpart was already present in the first Chandra observation of 2007/8, 25 days after the nova outburst. Initially, the source was bright $\left(L_{50} \sim 3 \times 10^{37} \mathrm{erg} \mathrm{s}^{-1}\right)$ but its equivalent luminosity declined fast (see Table 4 ). The nova exhibited a short SSS state with a duration of less than 100 days. The XMM-Newton EPIC PN spectrum therefore only contained few counts. It can be fitted with an absorbed blackbody model with $k T=66_{-24}^{+34} \mathrm{eV}$ and $N_{\mathrm{H}}=\left(0.9_{-0.8}^{+1.5}\right) \times 10^{21} \mathrm{~cm}^{-2}$, classifying this source as a SSS.

\subsubsection{M31N 2007-12d}

The optical nova was discovered independently by Henze et al. (2007) and Nishiyama \& Kabashima ${ }^{11}$ on 2007-12-17.58 UT. The accuracy of the time of the nova outburst is about 0.4 days,
9 See http://www.cfa.harvard.edu/iau/CBAT_M31.html\# $2007-02 b$
10 See extragalactic_novae/HET/
11 See http://www.cfa.harvard.edu/iau/CBAT_M31.html\# $2007-12 d$ 
based on a non-detection on 2007-12-17.19 UT (Burwitz et al. 2008; Henze et al. 2007). From our optical data obtained following the discovery by Henze et al. (2007) we estimate a very fast decline of the optical light curve ( $t_{2} \sim 4$ days). The object was classified as a He/N nova by Shafter (2007a), who reported strong and broad Balmer lines with a $F W H M$ for $\mathrm{H} \alpha$ of about $5500 \mathrm{~km} \mathrm{~s}^{-1}$. The line width implies a high ejection velocity of the nova envelope of about $2750 \mathrm{~km} \mathrm{~s}^{-1}$. Together with the fast decline of the optical light curve this implies a rapidly evolving nova.

A faint X-ray counterpart $\left(L_{50}=(2.8 \pm 0.8) \times 10^{36} \mathrm{erg} \mathrm{s}^{-1}\right)$ was visible in only one XMM-Newton observation about 22 days after outburst (see Table 4). The best-fit blackbody parameters for the low-count spectrum are $k T=66_{-28}^{+72} \mathrm{eV}$ and $N_{\mathrm{H}}=$ $\left(0.9_{-0.9}^{+9.9}\right) \times 10^{21} \mathrm{~cm}^{-2}$. Together with the hardness ratio criterion described in Sect. 2, these parameters allow us to classify the source as SSS.

Nothing was found at the position of M31N 2007-12d in $\mathrm{X}$-ray data on days 12 and 32 after outburst with upper limits of $L_{50} \lesssim 1.5 \times 10^{36} \mathrm{erg} \mathrm{s}^{-1}$. This indicates that the nova exhibited an extremely short SSS state of less than 20 days, supporting the interpretation of a very fast nova suggested by its optical properties. The speed of the nova evolution is remarkable, because it makes M31N 2007-12d not only the fastest SSS in our sample (see Table 9), but more so, the fastest of all novae known so far for which SSS emission was found. Its SSS duration is considerably shorter than those of the M 31 novae M31N 2007$11 \mathrm{a}$ and M31N 2007-12b (see Table 4) as well as those of the Galactic RNe RS Oph (about 60 days, Osborne et al. 2006) and even U Sco (about 28 days Schaefer et al. 2010; Schlegel et al. 2010). For all of these nova systems it was discussed that they might contain a massive WD (Kahabka et al. 1999; Hachisu et al. 2007; Henze et al. 2009b; Pietsch et al. 2011). The SSS duration of nova V2491 Cyg could be of comparable length, but its turnon time is longer (about 35 days, Page et al. 2010). In fact, so far V2491 Cyg and the two RNe mentioned above are the only Galactic novae with a short SSS phase of less than 100 days. Note that V2491 Cyg is discussed as a candidate RN in Page et al. (2010). Implications on the possible connection of $\mathrm{RNe}$ with M31N 2007-12d and other fast novae in our sample are discussed in Sect. 5.4.

\subsubsection{M31N 2008-05a}

The optical nova was discovered by Nishiyama \& Kabashima ${ }^{12}$ on 2008-05-15 and confirmed by Henze et al. (2008c) using $\mathrm{H} \alpha$ observations. Immler et al. (2008) report Swift UltraViolet/ Optical Telescope (UVOT) detections of the source on 2008-0526. An X-ray counterpart became visible 198 days after outburst in 2008/9 and its light curve, shown in Table 4, indicates significant variability by a factor of three or more. The turn-on time of the source is estimated as the midpoint between observations 9826 and 9827 . The object was still detected at the end of the 2008/9 campaign, therefore we can only give a lower limit for its X-ray turn-off (see Table 9).

The XMM-Newton EPIC spectrum of the source can be fitted by an absorbed blackbody model with $N_{\mathrm{H}}=\left(0.4_{-0.4}^{+1.8}\right) \times$ $10^{21} \mathrm{~cm}^{-2}$ and $k T=45_{-28}^{+25} \mathrm{eV}$, classifying this source as a SSS. Note that the formal best-fit $N_{\mathrm{H}}$ is lower than the Galactic foreground absorption of $\sim 6.7 \times 10^{20} \mathrm{~cm}^{-2}$ (but still compatible with it within the errors).

\footnotetext{
12 See http://www.cfa.harvard.edu/iau/CBAT_M31.html\# $2008-05 a$
}

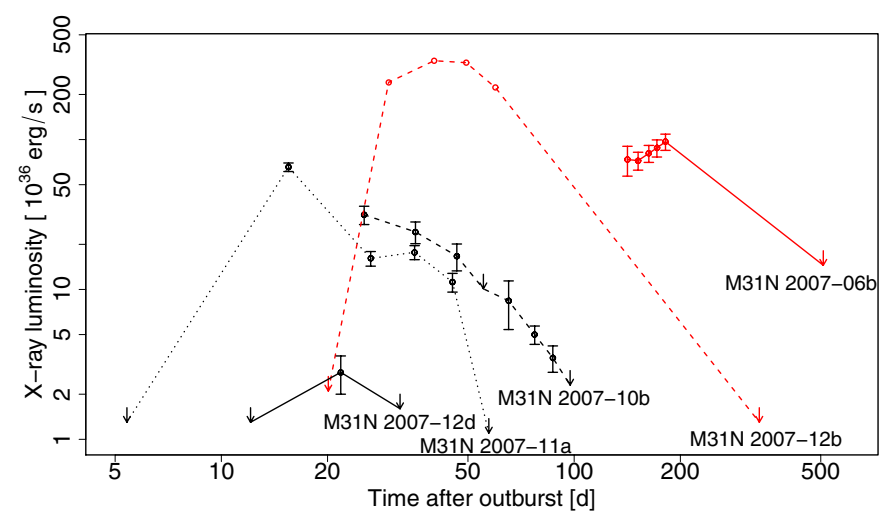

Fig. 3. X-ray light curves for all novae detected in 2007/8 and 2008/9 with short ( $<100$ days) SSS turn-on times. Note the logarithmic axes. Detections are indicated as open circles with error bars and upper limits as down-pointing arrows. For nova M31N 2007-12b the size of the error bars is smaller than the size of the symbols. Measurements for each nova are connected by lines, the style and colour of which distinguishes between the novae.

\subsection{9. $\mathrm{M} 31 \mathrm{~N} 2008-05 \mathrm{~b}$}

The optical nova was discovered by Nishiyama \& Kabashima ${ }^{13}$ on 2008-05-23. It was confirmed as a nova by Henze et al. (2008c), using $\mathrm{H} \alpha$ observations. Immler et al. (2008) report Swift UVOT detections on 2008-05-27. A faint X-ray counterpart is detected in four consecutive Chandra HRC-I observations 190-209 days after the outburst (see Table 4). The source is not detected in the two earlier Chandra observations, nor in the last two observations of the 2008/9 campaign. We estimate the turnon (turn-off) time as the midpoint between observations 9826 and 9827 (10838 and 0551690201). There is no significant variability during the duration of the X-ray visibility.

\subsubsection{0. $M 31 \mathrm{~N} 2008-06 a$}

The optical nova was discovered by Henze et al. (2008a) on 2008-06-14. An optical re-brightening of the object was observed on 2008-09-01 by Valcheva et al. (2008) (see also Ovcharov et al. 2009). Henze et al. (2008c) confirmed the object as a nova on the basis of $\mathrm{H} \alpha$ observations. A faint X-ray counterpart only appeared after 257 days in the very last observation of the 2008/9 campaign (see Table 4).

\subsection{Upper limits for non-detected X-ray emission of optical novae}

The two novae M31N 2001-01a and M31N 2005-02a were active SSSs until the end of the observations reported in Paper I. These sources are not detected any more (see Table 5). Upper limits for undetected novae with optical outburst from October 2006 till February 2008 and October 2007 till February 2009 are listed in Tables 6 and 7 for the 2007/8 and 2008/9 campaigns, respectively. We assume that nova M31N 2006-12d, which was reported by Hornoch ${ }^{14}$, was actually caused by a re-brightening of nova M31 2006-11b because the positions of both novae are nearly identical.

\footnotetext{
${ }^{13}$ See http://www.cfa.harvard.edu/iau/CBAT_M31.html\# 2008-05b

14 See http://www.cfa.harvard.edu/iau/CBAT_M31.html\# $2007-12 d$
} 


\subsection{Non-nova supersoft sources}

Additionally, we searched our XMM-Newton data for SSSs that do not have nova counterparts. This search was based on the hardness-ratio criterion described in Eq. (1) in Sect. 2. As a result, we found three sources that are already known SSSs. The light curves of these objects, all of which were also detected in Paper I, are given in Table 8 and their positions are shown in Fig. 1. In the following, we briefly describe the properties of these sources in our observations. We refer to the objects by their names in the catalogue of time-variable X-ray sources by Stiele et al. (2008), which contains all three sources.

Object XMMM31 J004252.5+411541 is a bright and persistent SSS (see Table 8) that has already been discovered with the Einstein Observatory (source 69 in Trinchieri \& Fabbiano 1991). It was extensively discussed by Trudolyubov \& Priedhorsky (2008), who reported X-ray pulsations with a period of about 217.7 s. They discussed the source as a magnetic WD that is steadily accreting and burning material. During our monitoring, the source was always detected with high luminosities $\left(L_{50}>10^{38} \mathrm{erg} \mathrm{s}^{-1}\right)$. Its light curve was variable by a factor of about two at most (see Table 8).

Object XMMM31 J004318.8+412017 was already discovered in early Chandra observations (Kaaret 2002; Kong et al. 2002). Williams et al. (2006) included it in their catalogue of transient X-ray sources in M 31 (named r3-8 there, from its designation in Kong et al. 2002) and discussed it as a Galactic foreground polar based on its soft spectrum. During our monitoring the source showed burst-like variability with luminosity increase by more than a factor of ten (see Table 8 ). This behaviour agrees with that described in Williams et al. (2006), who also reported four outbursts of the source.

Object XMMM31 J004318.7+411804 was reported as a previously unknown variable SSS by Stiele et al. (2008). They reported a maximum luminosity of $L_{50}=3.3 \times 10^{36} \mathrm{erg} \mathrm{s}^{-1}$ and classified the source as a candidate SSS. The object is detected in less than half of our monitoring observations (see Table 8). Its luminosity is only a few $10^{36} \mathrm{erg} \mathrm{s}^{-1}$ in most detections with the exception of two Chandra observations were it reaches $\sim 10^{37} \mathrm{erg} \mathrm{s}^{-1}$.

To summarise, in the two monitoring campaigns we found 17 X-ray nova counterparts in total. Thirteen of these sources have been classified as SSSs. Comparing this number to the three non-nova SSSs presented here, we can again confirm the finding of PFF2005 that optical novae are the major class of SSSs in the central part of M 31.

\section{Novae with X-ray counterpart in M 31 - the catalogue}

We compiled a catalogue of optical novae with an X-ray counterpart in M 31. This catalogue contains 60 objects and is mainly based $(\sim 85 \%)$ on the results of our monitoring campaigns for M 31 novae (presented here and in Paper I) and on our analysis of archival M 31 X-ray data (in PFF2005 and PHS2007). We searched the available literature and included further X-ray detections and measurements of M 31 novae reported by the following authors: Smirnova et al. (2006), Pietsch et al. (2007c), Voss et al. (2008), Orio \& Nelson (2008), Nelson et al. (2008a), and Stiele et al. (2010). To our knowledge, the catalogue contains all known M 31 novae with an X-ray counterpart discovered until the end of February 2009.

We did not include five apparent X-ray nova counterparts from a recent census of SSSs in M 31 (Orio et al. 2010), because we cannot confirm them in our data. These sources are the suggested X-ray counterparts of the novae M31N 200409b, M31N 2007-08b, M31N 2007-11c, M31N 2008-02a, and M31N 2008-06c (table 3 in Orio et al. 2010). In the cases of M31N 2007-11c and M31N 2008-06c the positions of the optical novae are relatively close to known non-SSS X-ray sources in the field (sources 388 and 405 from Pietsch et al. 2005b, respectively), which may have been mis-identified as nova counterparts.

Our catalogue is presented in Table 9 and contains the following information: (a) for the optical nova: the name, date of outburst detection, maximum observed magnitude in a certain filter (which is not necessarily the peak magnitude of the nova), $t_{2}$ decay time in the $R$ band, classification as belonging to the old/young stellar population, spectroscopic nova type in the classification scheme of Williams (1992), and expansion velocity of the ejected envelope as measured from the earliest optical spectrum (half of the $F W H M$ of the $\mathrm{H} \alpha$ line); (b) for the X-ray counterpart: the turn-on and turn-off times, a flag for SSS classification, the blackbody and WD atmosphere (solar and halo abundances) temperatures as inferred from the X-ray spectrum; (c) derived parameters: the ejected and burned masses as computed according to Sect. 5.3; (d) references. Note that not all parameter values are known for all objects. The full catalogue will be available in electronic form at the CDS.

\section{Discussion}

\subsection{Novae with long SSS states - sustained hydrogen burning through re-established accretion?}

In our monitoring we detected six X-ray counterparts of optical novae that were still active for about five to twelve years after the optical outburst. These novae are M31N 1996-08b, M31N 1997-11a, M31N 2001-10a, M31N 2004-05b (all described in Sect. 3.1), M31N 2003-08c, and M31N 2004-01b (see Sect. 3.2). They were all visible at the end of the 2008/9 monitoring for 12.5, 11.0, 7.4, 4.8, 5.5 and 5.2 years after the optical outburst, respectively (see Tables 2 and 4). Additionally, there is the possibility that faint SSSs, like XMMM31 J004318.7+411804 (see Sect. 3.4 and Table 8 ) that showed a light curve similar to that of M31N 2003-08c and was already detected in 2002 with XMM-Newton (Stiele et al. 2008), might be novae with a long SSS phase for which the optical outburst had been missed because of gaps in the optical monitoring.

Novae M31N 2003-08c and M31N 2004-01b are relatively faint sources $\left(L_{50} \sim 5 \times 10^{36} \mathrm{erg} \mathrm{s}^{-1}\right)$ that were detected for the first time in our 2007/8 and 2008/9 campaign, respectively. Both sources show variability by at least a factor of two (see Table 4) but there is no indication of a decline in luminosity over time. All of the remaining four novae were already detected by PHS2007 and were also found in our 2006/7 monitoring campaign described in Paper I. Of these four SSSs only M31N 1997-11a shows a declining light curve over the course of the three monitoring campaigns described in Paper I and in this work. The X-ray luminosity of the other three nova counterparts exhibits on average no significant change from 2007 to 2009.

For four of the novae (M31N 1997-11a, M31N 2004-05b, M31N 2003-08c and M31N 2004-01b) we found significant variability in their X-ray light curves on short time scales. The luminosity of M31N 1997-11a increased by about a factor of two from Chandra observation 8528 to 8529 (3689 days after outburst) and returned to its previous state in observation 8530 . 
For M31N 2004-05b we noticed a similar phenomenon between observations 8526, 8527 (1279 days after outburst), and 8528 (see Table 2). The observations are separated by about ten days (see Table 1). The variability of M31N 2003-08c is difficult to constrain, because the source is only slightly above the detection limit in most cases. The luminosity of nova M31N 2004-01b increased significantly towards the end of the 2008/9 campaign.

There are two scenarios in which the long SSS duration of these six novae can be explained. The first scenario assumes that the novae occurred on low-mass WDs, for which long post-nova SSS phases are expected (see e.g. Hachisu \& Kato 2006). For the duration of the H-burning, the bolometric luminosity of the nova should be constant while the photospheric surface shrinks and the effective temperature increases (see e.g. Gallagher \& Starrfield 1976; Hernanz 2005; Bode 2010). The slowly declining X-ray count rate of M31N 1997-11a therefore could be caused by a change in effective temperature.

In this context it is noteworthy that Shen et al. (2009) recently reported that for novae on He-core WDs with masses below $0.5 M_{\odot}$ the SSS phases can last for centuries or longer. These objects are expected to have low effective temperatures of $\sim 20 \mathrm{eV}$ and low X-ray luminosities. Shen et al. (2009) further note that CVs with He-core WDs have not yet been unambiguously discovered and that their post-nova SSS phase could be the easiest way to find them.

The second scenario assumes re-established hydrogen accretion in the binary system to prolong the nuclear burning on the WD surface that was initiated by the nova outburst. Such a setting was discussed by Ness et al. (2008) for the Galactic nova V723 Cas, which has the longest SSS phase known so far (more than 14 years in 2009; Schaefer \& Collazzi 2010). In this hierarchy, M31N 1996-08b is the current runner-up followed by M31N 1997-11a and the Galactic nova GQ Mus (SSS turn-off after 10 years; Schaefer \& Collazzi 2010; Shanley et al. 1995). For V723 Cas, Ness et al. (2008) reported an X-ray count rate variability within a factor of two of the mean, the physical reason of which is unclear.

A prolonged SSS phase caused by magnetically channelled, irradiation enhanced accretion onto a WD from its nearby companion star was also discussed recently by Schaefer \& Collazzi (2010) for their new "V1500 Cyg stars" subclass of Galactic novae. Unfortunately, the low X-ray and optical luminosities of the M 31 novae discussed here do not allow to test them for other characteristics of these subclass as short orbital periods, highly magnetised WDs and the behaviour of their quiescence magnitudes.

The scenarios discussed above underline the potential of M 31 novae with long SSS states for significant progress in the understanding of nova physics. Future monitoring observations of the M 31 central region would prove very useful in following the X-ray luminosity evolution of these novae and might allow a more decisive interpretation in favour of one scenario or the other.

\subsection{Correlations between nova parameters}

The nova SSS catalogue compiled in Table 9 was used to search for statistical correlations between the various X-ray and optical observables. Here we present correlations that were found between the following parameters: turn-on time $\left(t_{\text {on }}\right)$, turn-off time $\left(t_{\text {off }}\right)$, blackbody temperature $k T$ (all X-ray), $t_{2}$ decay time and expansion velocity of the ejected envelope (both optical). The correlations are shown in Figs. 5-9. To model the visible trends

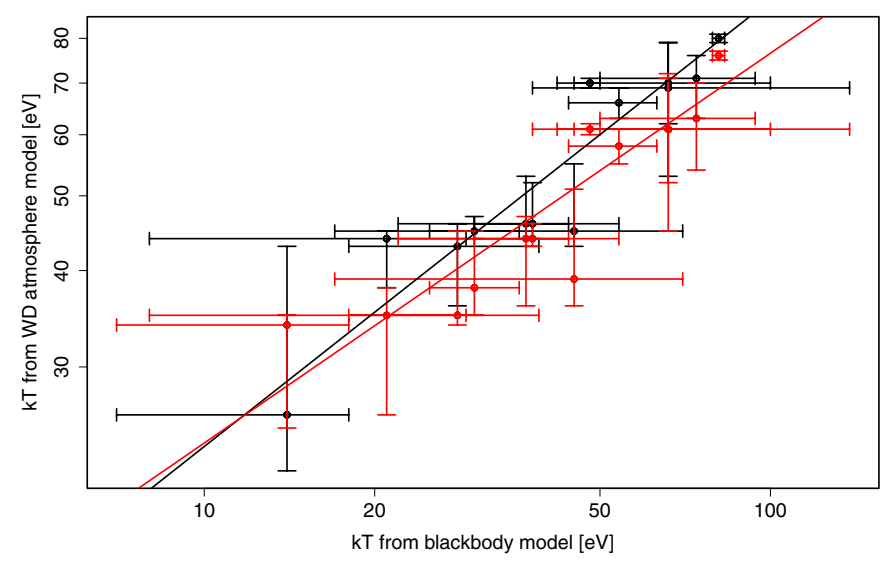

Fig. 4. Double logarithmic plot of blackbody temperature versus WD atmosphere temperatures using models with solar (black) and halo (red) abundances, including error bars and solid lines representing the best fit from a weighted regression.

we used a least-square fit with a power law, the results of which are given Eqs. (2)-(5).

We assume that the physical parameter mainly responsible for the various correlations is the WD mass. Also in optical studies the WD mass was found to be the dominating parameter (see e.g. Livio 1992; Della Valle \& Livio 1995; Della Valle 2002, and references therein). However, theoretical nova models show a more complicated picture (see e.g. Sala \& Hernanz 2005; Hachisu \& Kato 2006) and we include a note of caution about the physical interpretation of our correlations.

While a detailed interpretation of the observed correlations is beyond the scope of this paper we believe that our analysis revealed certain trends between different nova parameters that might be used as input for future theoretical models. Before we describe the discovered correlations, we discuss the connection between X-ray source temperatures derived using different spectral models.

\subsubsection{Blackbody vs. WD atmosphere temperatures}

Based on the subset of spectra analysed in this work and in Paper I (see Table 3), in Fig. 4 we plot the X-ray temperature derived from blackbody fits vs. the corresponding temperatures derived from NLTE WD atmosphere models. Despite large errors, the plot shows strong correlations between the different temperatures. This is confirmed by the Pearson correlation coefficients of $>0.9$ for both relations. While temperatures derived from WD atmosphere models are generally higher than blackbody temperatures, both approaches provide means to characterise broadly the effective temperature of SSSs.

This result confirms our initial assumption that blackbody temperatures can be used to characterise SSS temperatures of $\mathrm{CNe}$ (see Sect. 1). In the following statistical analysis, we will use blackbody temperatures because they are known for a larger set of novae in our catalogue.

\subsubsection{SSS turn-on time vs. turn-off time}

We plot the two X-ray time scales $t_{\text {on }}$ vs. $t_{\text {off }}$ in Fig. 5 . There is a trend correlating increasing turn-on times with increasing turnoff times. Note that because of the definition of both times it is not possible that $t_{\text {off }} \leq t_{\text {on }}$. The limiting case of $t_{\text {off }}=t_{\text {on }}$ is shown as a dotted black line in Fig. 5. However, the correlation that we 


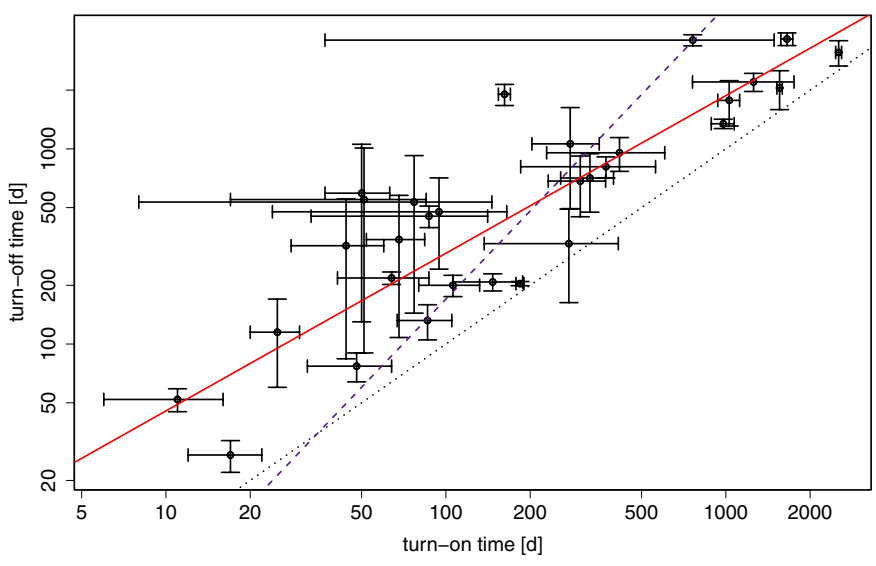

Fig. 5. Double logarithmic plot of turn-on time versus turn-off time (both in days after outburst) including error bars. The solid red line represents the best fit from a weighted regression. The dashed purple line shows the $t_{\text {off }}$ vs. $t_{\text {on }}$ relation of Hachisu \& Kato (2010). The dotted black line indicates the limiting case of $t_{\mathrm{off}}=t_{\mathrm{on}}$.

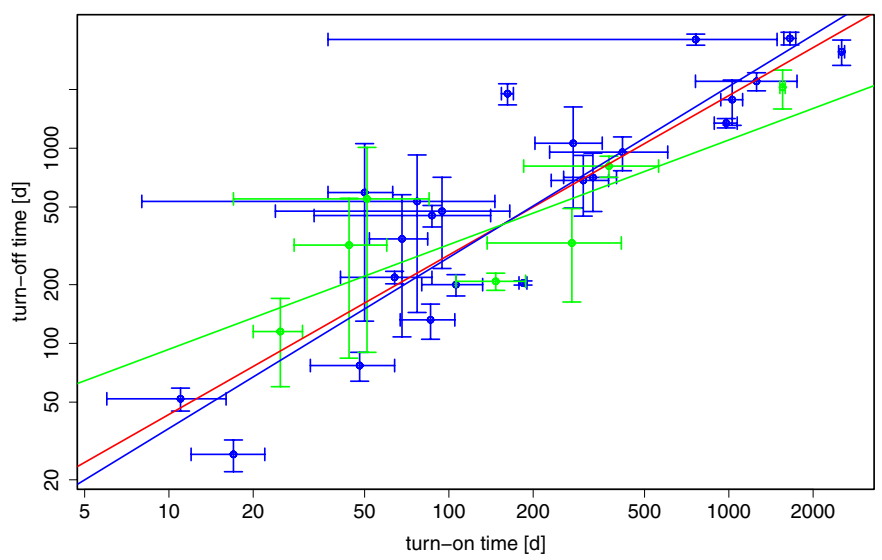

Fig. 6. Same as Fig. 5, here with different colours of symbols and bestfit lines for old novae (associated with bulge stellar population; blue) and young novae (disk population; green). The red line still shows the overall best fit.

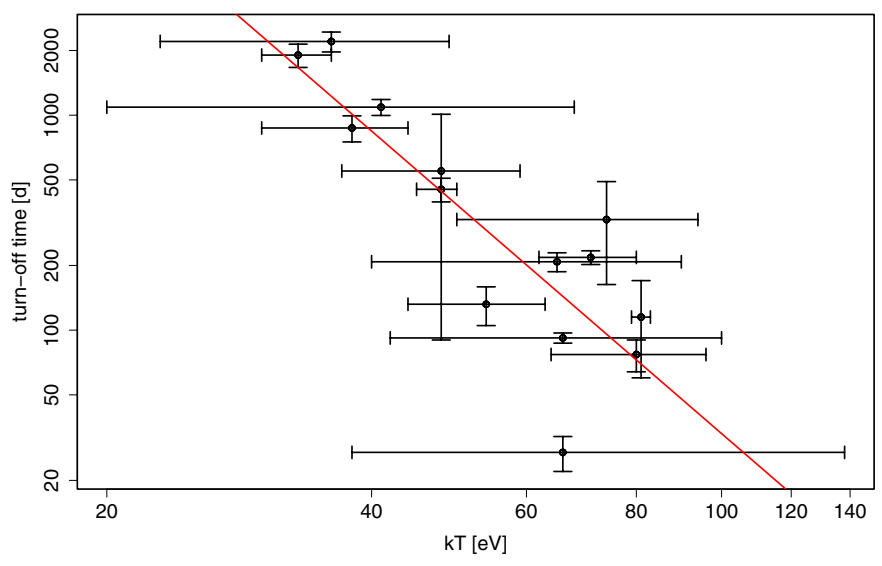

Fig. 7. Double logarithmic plot of blackbody temperature $(k T)$ in eV versus turn-off time in days after outburst including error bars. The red line represents the best fit from a weighted regression.

see is much more specific than $t_{\text {off }}>t_{\text {on }}$ and can be fitted with a powerlaw model. This model is shown as the solid red line in

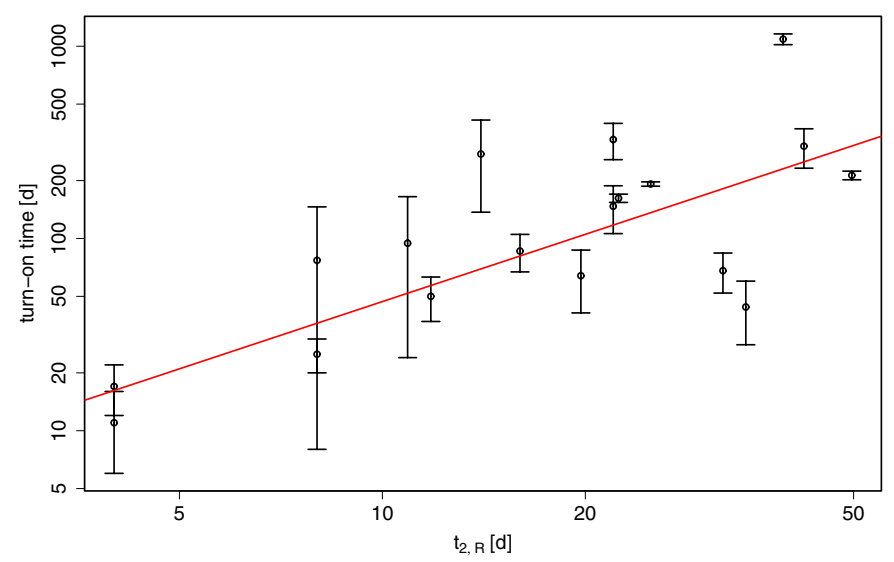

Fig. 8. Double logarithmic plot of optical decay time $t_{2, R}$ versus turn-on time (both in units of days after outburst) including error bars. The red line represents the best fit from a weighted regression.

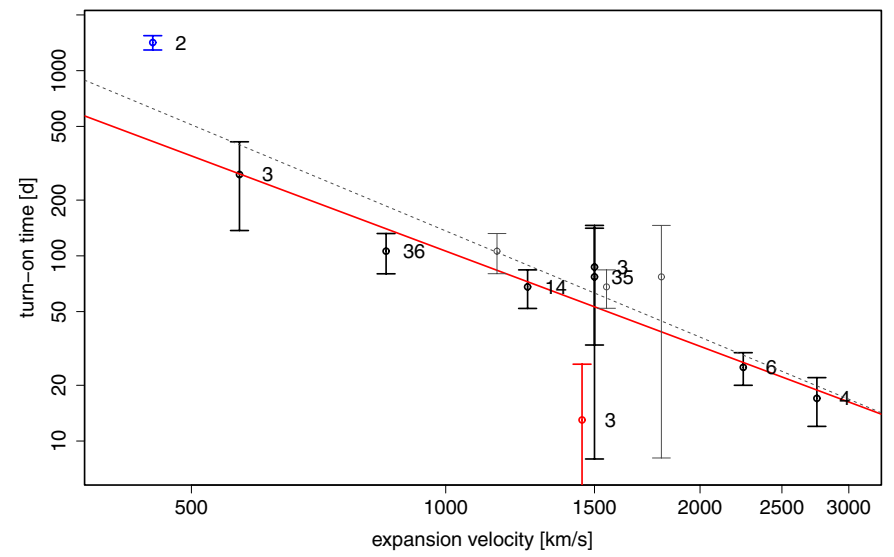

Fig. 9. Double logarithmic plot of expansion velocity in $\mathrm{km} \mathrm{s}^{-1}$ versus turn-on time in days after outburst including error bars. Annotations refer to the time elapsed in days between the outburst and when the optical spectrum was obtained. The red line indicates the best fit from a weighted regression. The red data point represents the atypical nova M31N 2007-10b. The blue data point is nova M31N 2003-08c for which the actual turn-on time is difficult to determine. The grey symbols visualise a generic shift of the expansion velocity by $+300 \mathrm{~km} \mathrm{~s}^{-1}$ for those novae for which spectra were taken more than ten days after outburst. The grey, dashed line shows the best fit after the shift.

Fig. 5 and defined by the following relation (both time scales in units of days after outburst):

$t_{\mathrm{off}}=10^{(0.9 \pm 0.2)} \cdot t_{\mathrm{on}}{ }^{(0.8 \pm 0.1)}$.

This dependence is significantly less steep than the relation between $t_{\mathrm{on}}$ and $t_{\mathrm{off}}$ inferred from a prediction formula of the SSS phase of novae recently published by Hachisu \& Kato (2010). From their Eqs. (25) and (26) one can derive that $t_{\mathrm{off}} \propto$ $t_{\mathrm{on}}^{1.5}$. This relation is shown as the dashed purple line in Fig. 5.

Separate modelling of novae from young and old stellar populations (see Sect. 5.5) indicates a difference in the model slope (see Fig. 6). The slope for old novae is $0.88 \pm 0.10$ (blue line in Fig. 6) and for young novae $0.54 \pm 0.18$ (green line in Fig. 6). However, this difference is significant only on the $1 \sigma$ level and an analysis of covariance does not show a significant impact of the type of population on the model. Because this result is strongly influenced by the small number of young novae, a larger sample is needed to study the difference further. 


\subsubsection{Blackbody temperature vs. X-ray time scales}

We plot the blackbody temperature $k T$ vs. the SSS turn-off time $t_{\text {off }}$ in Fig. 7. The figure shows an anti-correlation of these two X-ray parameters. We fitted this trend with a powerlaw, which is represented by the solid red line in Fig. 7. The fit indicates the following relation, where the turn-off time is given in units of days after outburst and the blackbody temperature in units of eV:

$t_{\text {off }}=10^{(8.1 \pm 0.8)} \cdot k T^{(-3.2 \pm 0.5)}$.

One should be aware that the absolute numbers given in this relation are based on blackbody fits, which do not describe the actual effective temperature of the source. However, in Sect. 5.2.1 above we found that a simple blackbody parameterisation seems capable of at least distinguishing between high-temperature and low-temperature SSSs. The general trend visible in Fig. 7 should therefore still be valid for effective temperatures as well.

In order to interpret this trend physically, we make use of a result from theoretical models which states that higher effective temperatures indicate a larger WD mass (see e.g. Fig. 7 in Sala \& Hernanz 2005). It is tempting to speculate that Eq. (3) represents a relation between WD mass and turn-off time. This relation might be similar to the one shown in Fig. 9a of Hachisu \& Kato (2006), where they plot the WD mass versus the time when hydrogen-burning ends (which is generally agreed on to correspond to the SSS turn-off time). The main caveat in establishing this relation is that Fig. 7 in Sala \& Hernanz (2005) is given for the maximum effective temperature, which is not the same as the blackbody $k T$ derived from our observations. First, because blackbody fits to supersoft spectra are not physically correct representations of an evolving WD atmosphere and are generally known to underestimate the source temperature. This problem is discussed above. Second, because our observations might not detect the SSS at its maximum temperature. However, Figs. 10 and 11 in Sala \& Hernanz (2005) show that novae evolve quickly through the low $k T$ phase in their SSS state and spend most of the time close to their maximum effective temperature. Therefore, it is possible that Fig. 7 shows a dependence of the turn-off time on the WD mass.

Finally, we repeated the regression for old novae and young novae separately (see Sect. 5.5), but did not find significant differences.

\subsubsection{Optical decay time vs. SSS turn-on time}

We plot the time of optical decay by two magnitudes in the $R$ band $\left(t_{2, R}\right)$ vs. the SSS turn-on time in Fig. 8. Note that there are only sufficient data to perform a statistical analysis from optical $R$ band light curves. The plot indicates a trend that is positively correlating the two parameters. We modelled this correlation with a powerlaw, which is indicated as a red line in Fig. 8. The model gives the following relation:

$t_{\mathrm{on}}=10^{(0.5 \pm 0.3)} \cdot t_{2, R}^{(1.2 \pm 0.2)}$.

Both time scales are in units of days after nova outburst. We therefore obtain a roughly linear relation between the two significant time scales in optical and X-ray. However, the scatter of the data points in Fig. 8 is relatively large and a few data points lie significantly off the powerlaw model. This behaviour might indicate a more complex relation between the two time scales that should be further examined in future studies using a larger nova sample.
Note that from their theoretical models, which are based on observations of Galactic novae, Hachisu \& Kato (2010) recently derived a relation between $t_{2}$ and the turn-on time that is also linear but much steeper (their Eq. (30) combined with (29)). This discrepancy might arise because Hachisu \& Kato (2010) used decay times in the emission-line-free optical y-band, whereas our results depend on $R$-band light curves. The continuum flux of a nova in this band is contaminated by the $\mathrm{H} \alpha$ emission line, which is the most prominent characteristic of a nova spectrum. Observers have used the fact that novae are longer visible in $\mathrm{H} \alpha$ since the work of Ciardullo et al. (1983).

\subsubsection{Optical expansion velocity vs. SSS turn-on time}

We plot the expansion velocity of the ejected envelope $\left(v_{\text {exp }}\right)$ as measured from optical spectra vs. the SSS turn-on time $\left(t_{\mathrm{on}}\right)$ in Fig. 9. This diagram includes all novae for which $v_{\text {exp }}$ is known and $t_{\text {on }}$ had been measured accurately enough. It shows an anticorrelation trend between both parameters.

Before we modelled this trend, we excluded two novae, which are colour-coded in Fig. 9: M31N 2003-08c (blue) and M31N 2007-10b (red). M31N 2003-08c has an equivalent luminosity close to our detection limit and is only found in half of the Chandra observations during both campaigns (see Table 4). Because of its faintness, the turn-on time of the source is difficult to determine. M31N 2007-10b seems to be a peculiar nova, since already Rau et al. (2007) noted the atypically low expansion velocity (for $\mathrm{He} / \mathrm{N}$ novae) of $1450 \pm 100 \mathrm{~km} \mathrm{~s}^{-1}$.

The powerlaw model that describes the correlation between the remaining data points is indicated by a red line in Fig. 9 and described by the following relation:

$t_{\mathrm{on}}=10^{(7.1 \pm 0.7)} \cdot v_{\exp }^{(-1.7 \pm 0.2)}$.

The turn-on time is given in units of days after nova outburst and the expansion velocity in units of $\mathrm{km} \mathrm{s}^{-1}$. This model is based only on a few objects for which both quantities are known. Nevertheless, the scatter is small and the correlation is strong, with a Pearson correlation coefficient of -0.96 and a non-correlation $p$-value of 0.0008 . Note that no expansion velocity uncertainties are available in the literature for all novae shown in Fig. 9 except for M31N 2006-09c $\left(570 \pm 45 \mathrm{~km} \mathrm{~s}^{-1}\right.$; Hatzidimitriou 2010, priv. comm.) and M31N 2007-10b (1450 \pm $100 \mathrm{~km} \mathrm{~s}^{-1}$; Rau et al. 2007).

Another caveat is that expansion velocities of novae are known to be time-dependent. Hatzidimitriou et al. (2007) found for the optical nova M31N 2005-09c (no SSS counterpart known) a decline in $\mathrm{H} \alpha F W H M$ of about $200 \mathrm{~km} \mathrm{~s}^{-1}$ within six days. However, there is no explicit relation between the expansion velocity and the time after nova outburst known so far. For four of the seven novae that we used to derive Eq. (5) the optical spectrum was taken within the first six days after outburst (see annotations in Fig. 9). Assuming a decline in expansion velocity over time, correcting for the delay in the remaining three novae with an arbitrary shift of $+300 \mathrm{~km} \mathrm{~s}^{-1}$ would slightly decrease the (negative) slope of the best fit (to $-1.9 \pm 0.3$ ), because these data points would move towards higher expansion velocities. This effect is visualised by the grey symbols and best-fit line in Fig. 9.

The implications of the trend visible in Fig. 9 are intuitively clear: higher expansion velocities should result in shorter turnon times of the SSS, because the ejected envelope becomes optically thin earlier. Equation (5) quantifies this correlation. It connects the two parameters needed to compute ejected masses 
(see Table 9) and could allow us to estimate these masses with higher accuracy (see Sect. 5.3). Furthermore, it could allow the estimation of the SSS turn-on time from the optical spectrum and therefore it could be an important tool for planning X-ray observations of optical novae.

\subsubsection{Physical interpretations - a note of caution}

At this point, a general note of caution is in order: theoretical models emphasise that the properties of a nova outburst are not only influenced by the mass of the WD, but also by its chemical composition (mainly the hydrogen content of the envelope, see e.g. Sala \& Hernanz 2005; Hachisu \& Kato 2006). This additional dependence is not accounted for in any of the simple power-law relationships presented here. However, according to the theoretical models, the impact of the chemical composition on the observed parameters appears to be considerably weaker than the influence of the WD mass: see e.g. Fig. 9 in Hachisu \& Kato (2006) and Fig. 7 in Sala \& Hernanz (2005). In particular, the effect of the chemical composition on $k T$ in Fig. 7 of Sala \& Hernanz (2005) (WD mass versus maximum effective temperature) seems to be in the same range $(\sim 15 \mathrm{eV})$ as the scatter and the error bars for $k T$ in our Fig. 7 (blackbody temperature versus turn-on time). Note that the scatter for the latter correlation also is about a factor of two larger than the dispersion of temperatures for comparing blackbody and WD atmosphere models in Fig. 4. Therefore, it is possible that most of the impact from parameters other than the WD mass on the correlations found in this work is still within the range of the (still relatively large) error bars. The possible extent to which the varying hydrogen content itself might be causing the observed scatter in the correlations might be an interesting question for further studies.

\subsection{Derived nova parameters}

In addition to the observed parameters of the optical nova and the X-ray counterpart, our catalogue (see Table 9) also contains the derived parameters: ejected hydrogen mass $\left(M_{\mathrm{ej}, \mathrm{H}}\right)$ and burned hydrogen mass $\left(M_{\text {burn,H }}\right)$.

The mass of hydrogen ejected in a nova outburst can be estimated from the turn-on time of the SSS and from the expansion velocity of the ejected material. Under the assumption of a spherical symmetric nova shell (Della Valle et al. 2002), the column density of hydrogen evolves with time as

$N_{\mathrm{H}}\left(\mathrm{cm}^{-2}\right)=M_{\mathrm{ej}, \mathrm{H}} /\left(\frac{4}{3} \pi m_{\mathrm{H}} v_{\mathrm{exp}}^{2} t^{2} f^{\prime}\right)$,

and the SSS turns on at $t=t_{\mathrm{on}}$ when $N_{\mathrm{H}}$ decreases to $\sim 10^{21} \mathrm{~cm}^{-2}$. Here, $m_{\mathrm{H}}=1.673 \times 10^{-24} \mathrm{~g}$ is the mass of the hydrogen atom and $f^{\prime} \sim 2.4$ a geometric correction factor (see Paper I). The newly found correlation between SSS turn-on time and expansion velocity given in Eq. (5) now allows us to eliminate the expansion velocity from this relation. We can therefore compute the ejected mass solely from the SSS turn-on time. This allows us to calculate more accurate mass and error range estimates for the vast majority of novae without an optical spectrum. Although Eq. (5) is only based on a few objects, it is an improvement compared to earlier work, where ejected masses had to be computed using a "typical" expansion velocity with unknown errors (see e.g. Paper I). Ejected hydrogen masses and error ranges in Table 9 were computed as follows, with $a=10^{8.4 \pm 0.2}$ being a correlation coefficient derived from inserting Eq. (5) into (6) for $t=t_{\mathrm{on}}$ :

$M_{\mathrm{ej}, \mathrm{H}}=N_{\mathrm{H}} \frac{4}{3} \pi m_{\mathrm{H}} f^{\prime} a t_{\mathrm{on}}{ }^{(1.1 \pm 0.1)}$.

This equation was also used for novae with known expansion velocities. Because of the tight correlation seen in Fig. 9, there are no big differences between computed and measured $v_{\exp }$ for these novae.

The amount of hydrogen mass burned on the WD surface is computed as in Paper I:

$M_{\mathrm{burn}, \mathrm{H}}=\left(L_{\mathrm{bol}} \cdot t_{\mathrm{off}}\right) /\left(X_{\mathrm{H}} \epsilon\right)$,

where $L_{\text {bol }}$ is the bolometric luminosity, $t_{\text {off }}$ the SSS turn-off time, $X_{\mathrm{H}}$ the hydrogen fraction of the burned material, and $\epsilon=5.98 \times 10^{18} \mathrm{erg} \mathrm{g}^{-1}$ (Sala \& Hernanz 2005). As in Paper I we use a constant bolometric luminosity of $3 \times 10^{4} L_{\odot}$ and a hydrogen mass fraction of $X_{\mathrm{H}}=0.5$ (for a discussion of these parameter values see Paper I).

Despite the uncertainties, the burned masses presented in Table 9 are within the range expected from models of stable envelopes with steady hydrogen burning (Sala \& Hernanz 2005; Tuchman \& Truran 1998). In general, the burned masses are about one order of magnitude smaller than the ejected masses, which for most novae are within the values predicted from hydrodynamical models of nova outbursts (José \& Hernanz 1998). Note that in the scenario of sustained H-burning through reestablished accretion (see Sect. 5.1) the burned masses computed for novae with long SSS states only constitute upper limits on the actual hydrogen mass left on the WD after the outburst.

\subsection{SSS phase duration in novae and the completeness of the $X$-ray monitoring}

In previous studies it had been noticed that only a minor fraction of novae in M 31 were actually observed as SSSs. While PHS2007 detected SSS emission from 11 out of 32 novae within about a year after optical outburst, in Paper I only 2 out of 25 novae were found in X-rays over a comparable time span. For the current work the corresponding numbers are 6 out of $28(2007 / 8)$ and 3 out of $23(2008 / 9)$ novae, respectively.

Based on current theoretical models, all novae are expected to display an SSS phase (see e.g. Hachisu \& Kato 2010). Therefore, the cause of the low percentage of actual detections remained an open question. It could be (a) caused by the inevitably incomplete observational coverage, or (b) by some inadequacy (or incompleteness) of the theoretical models.

Using our nova SSSs catalogue (see Table 9), we could test scenario (a) for the first time. This study strongly benefited from the substantial number of optical novae found in M 31 over the last 15 years and from the large number of archival and monitoring X-ray observations covering the M 31 central area.

The main steps of our approach were the following: We took the observed mass distribution of WDs in novae as known from theoretical work, converted it into a distribution of SSS turnon times, "convolved" it with our observational coverage and compared the expected number of detections to the actual observed number of SSS novae using a Monte Carlo Markov chain (MCMC) method. The entire procedure is explained in detail in the paragraphs below and the results are subsequently discussed.

Our starting point was the observed mass function of WDs in CNe, which we computed based on Truran \& Livio (1986). This approach assumes a Salpeter IMF $\Phi(M) \propto M^{-2.35}$ 


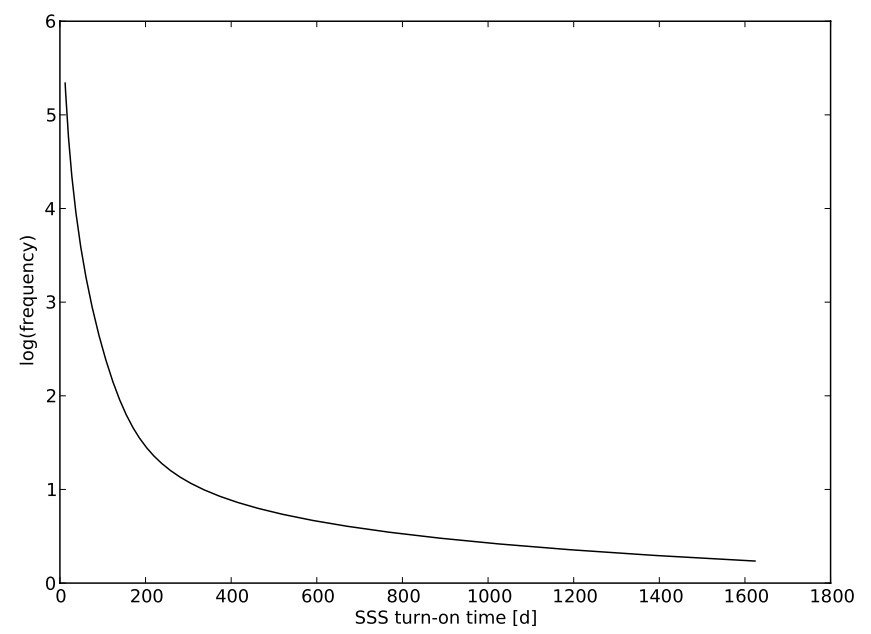

Fig. 10. Expected logarithmic frequency of SSS turn-on times in $\mathrm{CNe}$ based on Truran \& Livio (1986) and Hachisu \& Kato (2006).

(Salpeter 1955) for the WD progenitors and computes the nova recurrence frequency as $\nu_{\mathrm{rec}} \propto M_{\mathrm{WD}} / R_{\mathrm{WD}}^{4}$ based on the critical envelope mass needed to trigger the nova explosion (for more details see Truran \& Livio 1986). The intrinsic mass distribution of WDs has its strongest peak at low masses around $0.6 M_{\odot}$ (see e.g. Catalán et al. 2008, and references therein). However, the WD mass dependence of the recurrence frequency leads to a much higher observed frequency of high mass WDs in CNe (see also Ritter et al. 1991).

In order to translate the expected observed WD mass distribution into an expected observed distribution of SSS turn-on times we used the theoretical models of Hachisu \& Kato (2006). These authors computed SSS turn-on and turn-off times for different WD masses and chemical compositions based on a freefree emission model. We used their model values for CO WDs and $X_{\mathrm{H}}=0.45$ (their Table $4 ; t_{\text {wind }}$ corresponds to $t_{\mathrm{on}}$ ) because it is closest to our assumptions in this paper. Moreover, Fig. 9 in Hachisu \& Kato (2006) shows that the impact of choosing different WD chemical parameters is not huge and thus would not introduce significant errors to our analysis. The values between the grid points in their model were interpolated using a polynomial function. In Fig. 10 we show the resulting expected observed distribution of SSS turn-on times, which is clearly dominated by fast SSSs.

Our method is based on all optical novae discovered in M 31 from 1995 until February 2009, the end of our 2008/9 campaign. From this data set we selected all novae in the field of view of our XMM-Newton/Chandra M 31 centre monitoring. To account for the fact that XMM-Newton observations suffer from source confusion in the innermost arcminute around the M 31 centre, we exclude novae from this region. The resulting sample consists of 206 objects. Using a Monte Carlo method, we randomly selected SSS turn-on times for a certain fraction $x$ of these novae based on the expected turn-on distribution described above. To compute the associated SSS turn-off times, we used the correlation found in our catalogue (see Eq. (2)). This resulted in a certain time span of SSS visibility for the selected fraction of novae. The fraction $x$ was the free parameter to be optimised by the MCMC, thereby allowing us to test the scenario (a) outlined above.

We now made use of the large number of M 31 centre observations to estimate discovery rates for the simulated SSSs. These observations include the two monitoring campaigns this paper is based on (see Table 1), the monitoring campaign

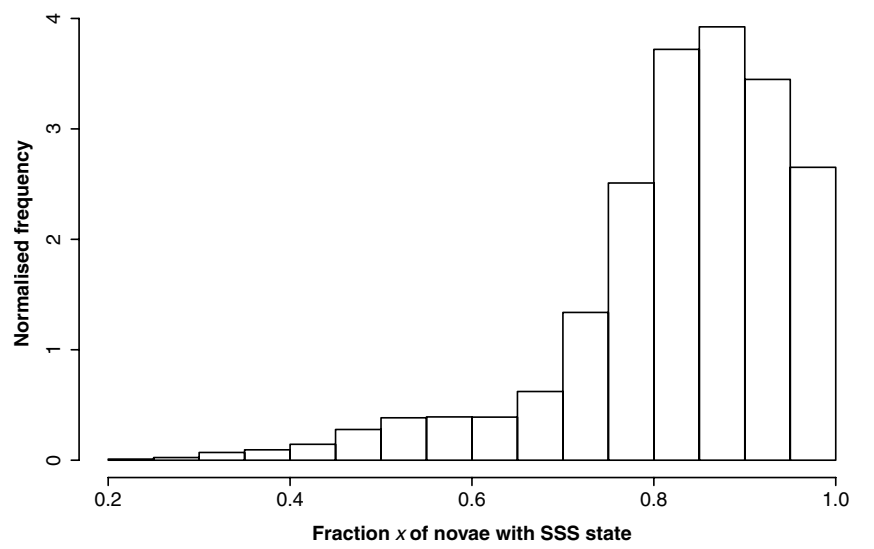

Fig. 11. Result from a MCMC simulation based on the intrinsic distribution of SSS turn-on times for novae in M 31. Shown is the frequency distribution of the intrinsic fraction $x$ of novae with SSS emission that would be needed to cause the detections in our observation campaigns.

described in Paper I (their Table 1) and the archival XMMNewton and Chandra HRC-I observations analysed by PHS2007 (their Tables 2 and 3) and PFF2005. From PFF2005, we only used the XMM-Newton centre observations (c1-c4 in Table 1 of Pietsch et al. 2005b) and the long Chandra observations 1912 and 1575. The other observations analysed by PFF2005 were either not pointed at the M 31 centre or only had a short exposure time, and are therefore not useful for this simulation. In total, there are 48 individual observations covering 8.7 years. In the context of the simulation, the SSS counterpart of a nova is defined as detected if there is at least one observation between SSS turn-on time and turn-off time. This somewhat ideal assumption is nonetheless justified because SSS counterparts of novae are expected to have a bolometric luminosity close to the Eddington limit of the WD (Hernanz 2005), which is between $6 \times 10^{37} \mathrm{erg} \mathrm{s}^{-1}$ and $2 \times 10^{38} \mathrm{erg} \mathrm{s}^{-1}$ for WD masses between 0.5 and 1.4 solar masses. If we assume that most of that luminosity is emitted in the soft X-ray band and take into account our typical observational sensitivities of a few $10^{36} \mathrm{erg} \mathrm{s}^{-1}$ (see e.g. Table 7), our actual detection efficiency should be close to $100 \%$.

Using the MCMC, SSS turn-on and turn-off times were determined for all novae and the number of sources with detected SSS phase was measured for each of the five campaigns separately. These predicted numbers were then compared to the actual number of novae detected as SSSs in PFF2005 (10) PHS2007 (14), Paper I (8), 2007/8 (11) and 2008/9 (9) for the epoch and spatial region selected above. The deviations between prediction and observation were summed up quadratically to create an error for the estimate. The Markov chain is governed by a Metropolis algorithm (Metropolis et al. 1953) that seeks to minimise this error by modifying the fraction $x$ of novae that the turn-on times are assigned to. The random-walk nature of the MCMC allowed us to find the fraction associated with the minimum error and to sample the parameter space around it.

We show the result of the simulation in Fig. 11, where the frequency distribution of the SSS fraction $x$ is plotted. This graph shows that our observational findings are consistent with the assumption that all novae exhibit a SSS stage and that the incomplete observational coverage is the reason for the detection of only a part of them. This result further highlights the importance of novae with high mass WDs and very short SSS turnon times, which was first found by PHS2007. According to our simulations, the intrinsic observed WD mass function strongly 
favours novae with short SSS states, which are expected to account for the majority of the observed sources.

Indeed, we find already five novae with fast SSS turn-on times in the 2007/8 campaign. Their light curves are shown in Fig. 3. A particularly interesting object from this sample is M31N 2007-12d. This nova showed a very short SSS phase and was only detected as a faint source in one observation (see Table 4). An object like this would have been very likely missed in a sparser sampling. Even in our ten day monitoring it is very close to the detection limit. Therefore, M31N 2007-12d might indicate the lower limit of SSS durations that we are still able to detect with the monitoring strategy applied in this paper.

\subsection{Nova population study}

The existence of two different nova populations, associated with the bulge and disk of a spiral galaxy, was first postulated based on optical data of Galactic novae (Duerbeck 1990; Della Valle et al. 1992; Della Valle \& Livio 1998). Slow Fe II novae were found to be located preferably in the bulge, whereas the faster $\mathrm{He} / \mathrm{N}$ novae (see Williams 1992, for the spectral classification) mostly belong to the disk. This suggests an association of fast (slow) novae with the overall young (old) stellar population in the disk (bulge). The size and spatial coverage of our nova catalogue, presented in Table 9, for the first time allowed us to investigate the $\mathrm{X}$-ray properties of novae belonging to these two populations in M 31 .

Our approach was two-fold. First, we used geometric parameters to distinguish between bulge (old population) and disk (young population) and examined the differences in the distributions of the individual nova parameters for both subsets. Second, we used the X-ray parameters of all novae to divide them into two groups of novae with massive or less massive WDs and tested their geometric distributions. While the first method assumes the existence of two different nova populations, as suggested from optical data, the second method is independent of this assumption. By comparing both approaches, we hoped to correct for selection biases that either of them might introduce. However, in both approaches we applied a geometric criterion and have to be aware that because of the high inclination of M 31 $\left(77.5^{\circ}\right.$; see e.g. Beaton et al. 2007) a significant number of novae occurring in the disk will be projected onto the bulge.

For the first approach, in order to assign a nova to one of the two populations, we used an entirely geometrical criterion. We followed the work of Beaton et al. (2007), who analysed NIR images of M 31, and defined the projected M 31 bulge as an ellipse with a semi-major axis of $700^{\prime \prime}$, an ellipticity of 0.5 , and a position angle of $\sim 50^{\circ}$. The boundary between the bulge and disk regions is marked by a grey ellipse in Fig. 12. In the context of this approach, old novae are defined as situated within this boundary and young novae lie outside of it. In Fig. 12 we show the positions of X-ray detected old novae as white and young novae as black crosses, respectively. Note that we classified nova M31N 2007-06b as an "old nova", the position of which is indicated by the only white cross outside the grey ellipse in Fig. 12. This object was found to be a nova in an M 31 globular cluster (see Shafter \& Quimby 2007; Henze et al. 2009a), and therefore belongs to a stellar population similar to the one dominating in the bulge.

We checked the observed distributions of the X-ray parameters given in Table 9 for dependencies on the classification as old or young nova. We found significant differences only for the blackbody temperature $k T$. In Fig. 13 we show the individual $k T$ distributions for young and old novae, respectively. Both

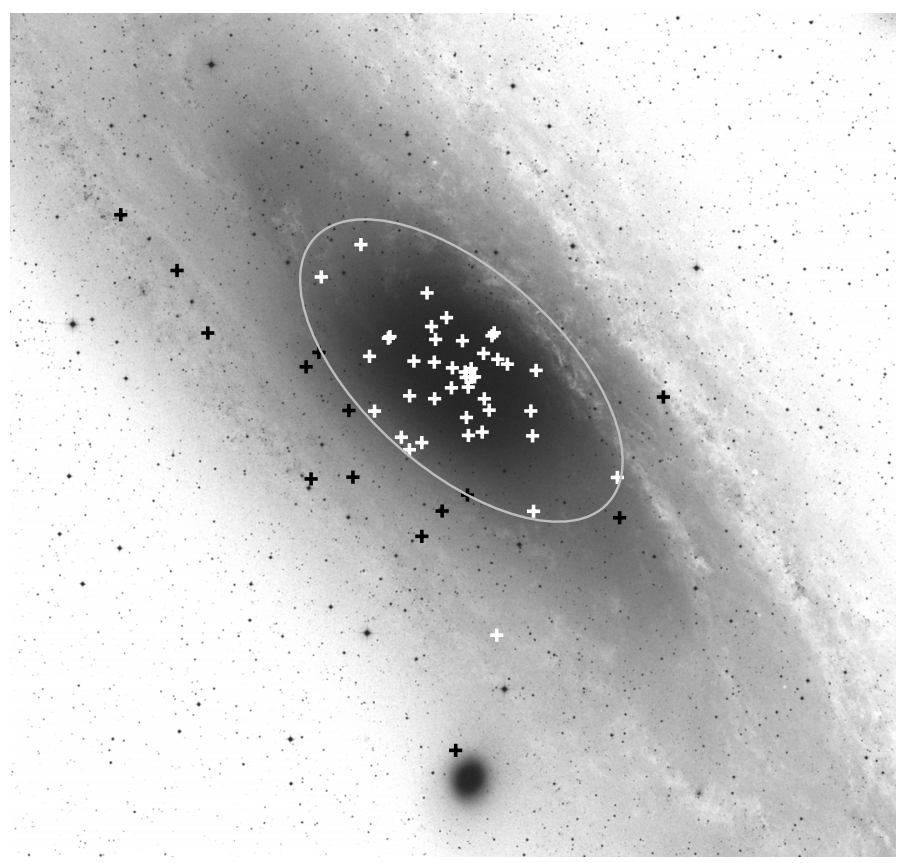

Fig. 12. Location of the M 31 old (white) and young novae (black) overlaid on a DSS2-R image. The grey ellipse marks the boundary between the M 31 bulge and the disk that was used in this work. See Sect. 5.5 for an explanation of the classification. Only four of the 60 nova SSSs from Table 9 are outside this image.
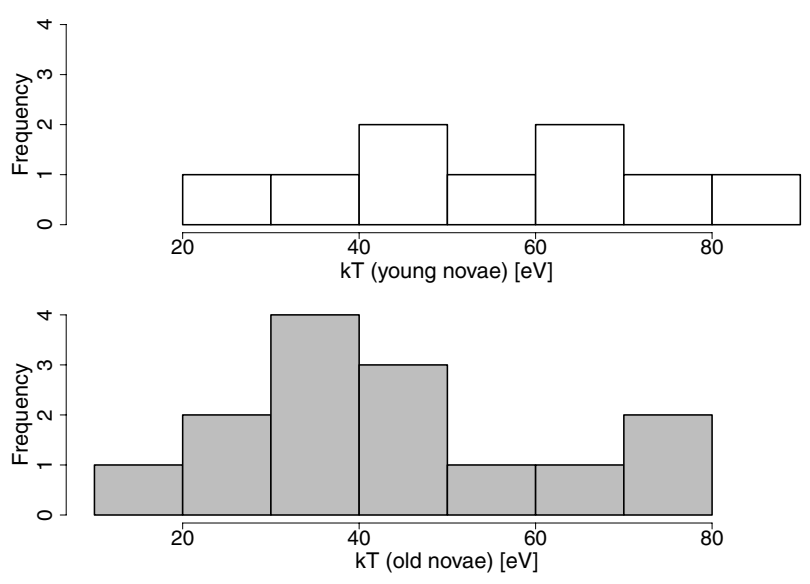

Fig. 13. Distribution of effective (blackbody) temperature $k T$ for young novae (white/upper panel) and old novae (grey/lower panel), respectively.

distributions are compatible with a Gaussian distribution on the $1 \sigma$ level (Kolmogorov-Smirnov (KS) test), but have different mean values of $44 \mathrm{eV}$ (old) and $56 \mathrm{eV}$ (young). We performed a two-sample t-test, which gives a $p$-value of $\sim 0.12$ for $20 \mathrm{de}$ grees of freedom, resulting in an $\sim 88 \%$ probability that the two distributions are different. An F-test shows that both variances are equal on the $1 \sigma$ level $(p$-value $=0.84)$ and that the t-test is therefore justified. However, the samples of both old and young novae with measured temperatures (14 and 9) are small. Indeed, statistical power calculations show that if the measured difference in blackbody $k T$ between the samples actually is $12 \mathrm{eV}$, we would need at least 32 novae in each group to see a difference on the $95 \%$ confidence level (with a power of 0.8 ).

We stress again that blackbody $k T$ values are only a simple parameterisation of the SSS spectrum and are not an accurate 


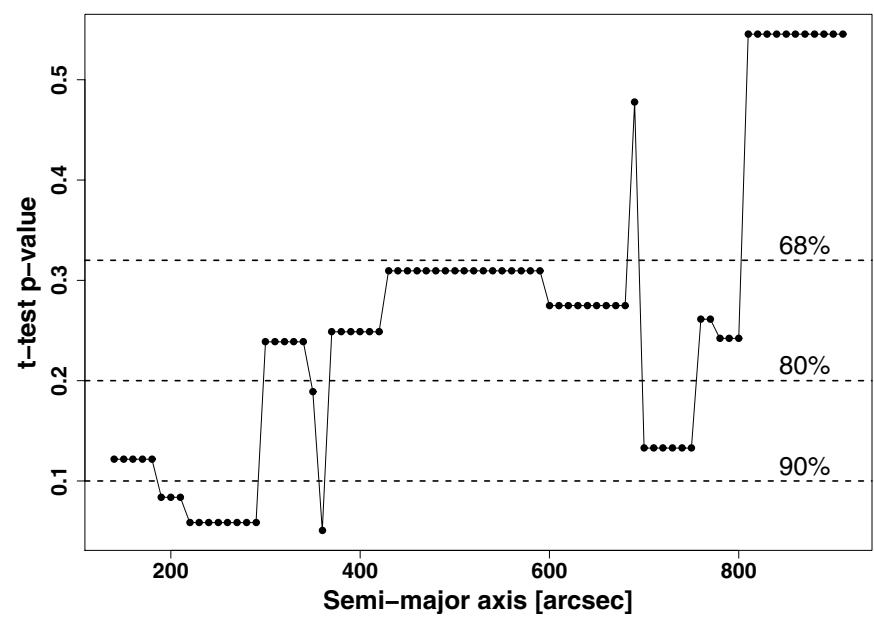

Fig. 14. Two-sample t-test $p$-values for the blackbody $k T$ distributions of old and young novae. The abscissa gives the semi-major axis of the "bulge" region which is defined to contain the old novae. The solid line connects the solid circles of the data points for better readability. Dashed lines show three acceptance levels for the t-test.

representation of the actual photospheric temperature of the source. However, in Sect. 5.2.1 we found that blackbody fits seem to be able to distinguish at least broadly between low and high-temperature sources.

In order to examine how much the result obtained above depends on our selection of the boundary between bulge and disk, we tested the method outlined above for different bulge extensions. In Fig. 14 we display the results depending on the semi-major axis of the bulge region. For this computation, we took into account the effect of changing ellipticity in the NIR isophotes of M 31 (see Beaton et al. 2007, their Fig. 3). The figure shows the t-test $p$-values for the blackbody $k T$ distributions of the respective groups of old and young novae. We can see that only for $200^{\prime \prime} \ldots 300^{\prime \prime}$ and $\sim 700^{\prime \prime}$ "bulges" there really is a significant difference on the $\sim 90 \%$ level.

In the second approach, we corrected the nova coordinates for the inclination of M 31 and computed M 31-centric distances for all objects. Of course, the effect of the projection of disk novae onto the bulge must also be kept in mind here. The X-ray parameter measured for most novae is the turn-on time. Similar to Sect. 5.4, we used the connection between the WD mass and the turn-on time inferred from Hachisu \& Kato (2006) to distinguish between high- and low mass WDs. We defined high mass WDs as $M_{\mathrm{WD}} \gtrsim 1.2 M_{\odot}$, which corresponds to $t_{\mathrm{on}} \lesssim 100 \mathrm{~d}$, and low mass WDs as $M_{\mathrm{WD}} \lesssim 0.7 M_{\odot}$, corresponding to $t_{\mathrm{on}} \gtrsim$ $500 \mathrm{~d}$. With this selection we sampled both the high end of the WD mass distribution, which dominates the observed mass distribution of WDs in nova systems, and the region around the peak of the observed mass distribution of single WDs at $\sim 0.6 M_{\odot}$ (see Sect. 5.4). Again, we only used novae whose turn-on time is determined accurately enough. In Fig. 15 we give the distributions of the distance from the M 31 centre for both groups. It shows that novae with low mass WDs seem to be more concentrated towards the centre of the galaxy than those with high mass WDs. A KS two-sample test shows that both distributions are significantly different on the 95\% level ( $p$-value of 0.044 ).

Clearly, these results have to be interpreted carefully. Firstly, because of the relatively small size of the sample of SSS novae, and secondly, because of projection effects. Despite these caveats our two approaches used two different geometric criteria as well as two different X-ray parameters and reached similar
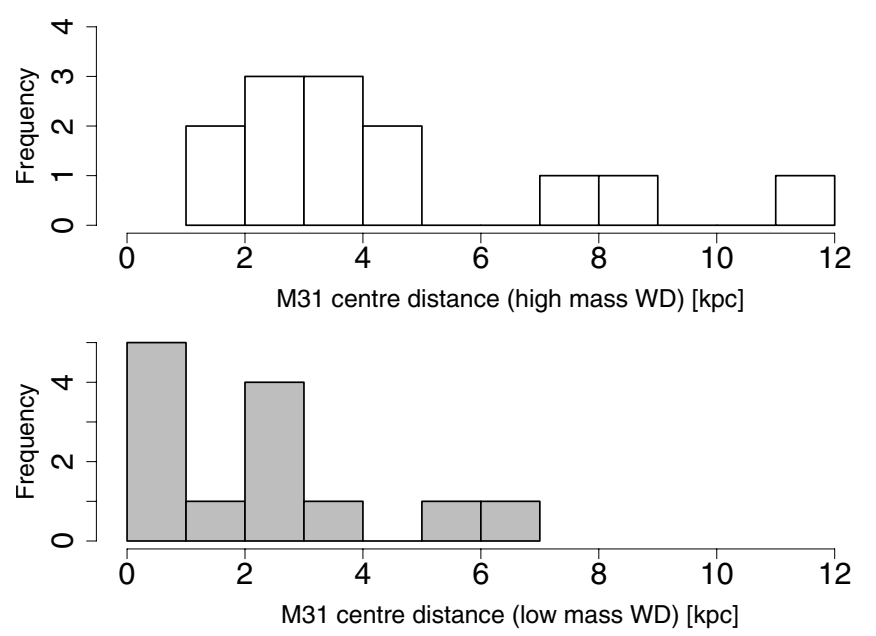

Fig. 15. Distribution of inclination-corrected M 31-centric distances for novae with high mass (upper panel, white) and low mass (lower panel, grey) WDs. Distances are given in kpc, assuming a distance to M 31 of $780 \mathrm{kpc}$ and are not corrected for projection effects.
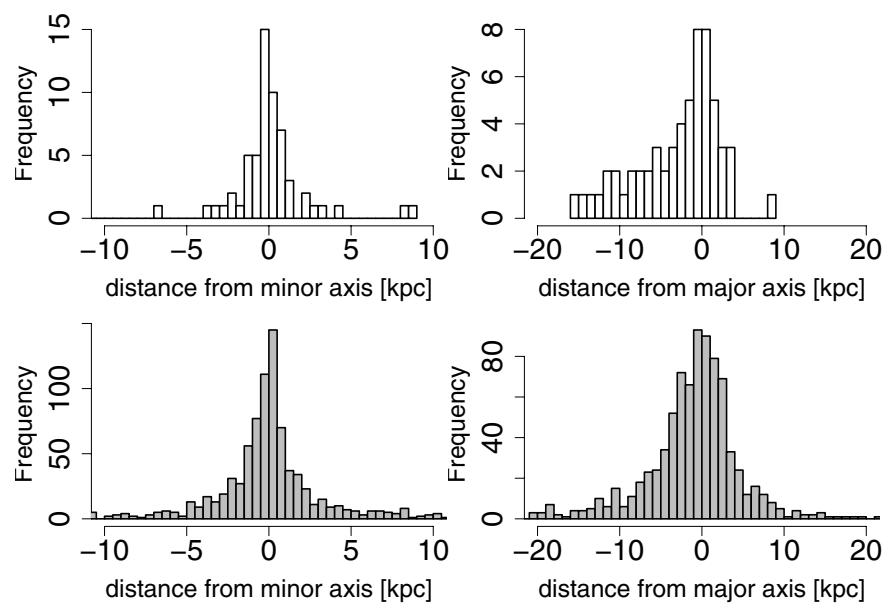

Fig. 16. Distributions of inclination-corrected nova positions with respect to the minor axis (left) and the major axis (right) of $\mathrm{M} 31$ for all known optical novae (lower panels, grey) and for novae with detected SSS counterpart (upper panels, white). Coordinate units are kpc, assuming a distance to M 31 of $780 \mathrm{kpc}$, and are not corrected for projection effects. Negative values correspond to positions in the south-east (far) part of M 31.

results. Both approaches therefore gave a first hint that in the $\mathrm{X}$-ray regime as well there are two distinct populations of novae that can be associated with the different stellar environments of bulge and disk. Additional observations are needed, in particular of the relatively neglected M 31 disk, to examine if our results can be verified.

\subsection{Asymmetric distribution of novae in X-rays}

Another observational result that we derive from Fig. 12 is that there appears to be an asymmetry in the spatial distribution of nova SSSs with respect to the major axis of M 31. There were more objects found on the (far) south-east side of M 31 than on the (near) north-west side. No such asymmetry was found in the spatial distribution with respect to the minor axis (north-east vs. south-west). In Fig. 16 we plot both distributions in the upper panels and compare them in the lower panels to the equivalent 
distributions for all known optical novae in M 31. The overall distributions appear symmetric with respect to both axes. We carried out KS tests that confirm the visual impression. The hypothesis that the distribution of nova SSS positions is symmetric with respect to the major axis (upper right panel in Fig. 16) is rejected on the $99 \%$ confidence level. On the other hand, the symmetry of the nova SSSs positions with respect to the minor axis (upper left panel in Fig. 16) is confirmed on the 85\% level. Projection effects might influence this result slightly, but cannot explain the asymmetry.

The cause of this asymmetry might be the fact that the northwest side of the galaxy is largely seen through the gas and dust in the spiral arms of M 31 (see Walterbos \& Kennicutt 1988). Bogdán \& Gilfanov (2008) found a similar asymmetry for the diffuse soft X-ray emission of the M 31 bulge. Based on a microlensing survey, An et al. (2004) found that also in the optical "all the variable star distributions are asymmetric in the sense that the far side (or south-east) is brighter or has more detected objects than the near side (or north-west)". They concluded that extinction within M 31 is the reason for this behaviour. For the entire optical nova sample, we see a slight asymmetry with respect to the major axis (lower right panel in Fig. 16). The median of the distribution is shifted to the south-east (negative values in Fig. 16) by about $0.5 \mathrm{kpc}$. However, this effect is significantly weaker than the asymmetry of the nova SSSs distribution.

Therefore, we assume that extinction within M 31 is the main reason for the observed asymmetry in nova SSSs positions with respect to the major axis of M 31. Currently, the number of known nova SSSs is not sufficient to allow us to quantitatively estimate the influence of the nova position in M 31 on the SSS detection probability. Note that this relation, once it is derived, would be useful to implement in the simulation method described in Sect. 5.4.

\section{Summary}

We described the second and third campaign of our dedicated Xray monitoring for counterparts of classical novae in $M 31$ during autumn and winter 2007/8 and 2008/9. We detected $17 \mathrm{X}$-ray counterparts of CNe in M 31 in total, 13 of which were not found in earlier studies. The remaining four novae are still active SSSs for $12.5,11.0,7.4$, and 4.8 years after the optical outburst and we discussed whether these long SSS phases might be sustained by re-established accretion onto the WD. During both monitoring campaigns there were only three (known) SSSs detected that do not have an optical counterpart, compared to 13 out of the 17 nova counterparts that we could classify as SSSs. Once more, this result confirms the statement of PFF2005 that novae are the major class of SSSs in the central region of M 31.

Several of the novae found in our monitoring display a short SSSs phase of less than 100 days. Based on the theoretically predicted observable WD mass distribution in novae, we conducted a simulation on the detectability of nova SSS states. This simulation showed that short SSSs, which are dominating the observed nova population, could account for the high number of novae that were not detected as SSS in this and previous studies.

Based on the results from this work and previous, partly archival studies, we compiled a catalogue of all novae with a SSS state in M 31 known so far. This catalogue contains 60 sources in total and for most of them several optical and Xray parameters are known. We used this catalogue to search for statistical correlations between these properties. Relationships were found between the parameters: turn-on time, turn-off time, blackbody temperature $k T$ (all X-ray), $t_{2}$ decay time and expansion velocity of the ejected envelope (both optical). We fitted our SSS spectra with NLTE WD atmosphere models and found that the temperature distribution derived from these models can be adequately described using the corresponding blackbody temperatures. We derived the values for the masses that were ejected and burned during the nova process and included them in our catalogue. Furthermore, we analysed the geometric distribution of nova SSSs in M 31 for different ranges of Xray parameters. Thereby, a first hint was found that in the X-ray regime there might be two distinct populations of novae that are associated with the bulge and the disk of M 31. A similar interpretation of optical data is already the subject of discussion for almost two decades now.

We emphasise that the effort for a regular monitoring of M 31 dedicated to novae has led to the point where for the first time a sample of nova SSSs exists that is becoming sufficiently extensive to be studied with statistical methods. The trends that we derive from this sample in this work might help to gain deeper insights into the physics of the nova process. A study of nova SSSs as a population, as presented here, can only be performed in M 31. Therefore, it is important to continue the regular monitoring of this galaxy to examine if our first results can be confirmed with an increased sample of nova SSSs.

Acknowledgements. We are grateful to Felix Kaduk for his help in compiling the nova SSS catalogue and to Kamil Hornoch for providing us some of his unpublished nova light curves. The anonymous referee is acknowledged for his constructive comments and suggestions. The X-ray work is based in part on observations with XMM-Newton, an ESA Science Mission with instruments and contributions directly funded by ESA Member States and NASA. The $X M M$-Newton project is supported by the Bundesministerium für Wirtschaft und Technologie/Deutsches Zentrum für Luft- und Raumfahrt (BMWI/DLR FKZ 50 OX 0001) and the Max-Planck Society. M. Henze acknowledges support from the BMWI/DLR, FKZ 50 OR 0405. A.R. acknowledges support from SAO grant GO9-0024X. M. Hernanz acknowledges support from grants AYA2008-01839 and 2009-SGR-315. G.S. acknowledges support from grants AYA2008-04211C02-01 and AYA2007-66256.

\section{References}

Alksnis, A., Smirnova, O., \& Zharova, A. V. 2008, Astron. Lett., 34, 563 An, J. H., Evans, N. W., Hewett, P., et al. 2004, MNRAS, 351, 1071 Ansari, R., Aurière, M., Baillon, P., et al. 2004, A\&A, 421, 509

Arnaud, K. A. 1996, in Astronomical Data Analysis Software and Systems V, ed. G. H. Jacoby, \& J. Barnes, ASP Conf. Ser., 101, 17

Baernbantner, O., \& Riffeser, A. 2006, The Astronomer's Telegram, 808, 1 Balucinska-Church, M., \& McCammon, D. 1992, ApJ, 400, 699

Beaton, R. L., Majewski, S. R., Guhathakurta, P., et al. 2007, ApJ, 658, L91

Bode, M. F. 2010, Astron. Nachr., 331, 160

Bode, M. F., Darnley, M. J., Shafter, A. W., et al. 2009, ApJ, 705, 1056

Bogdán, Á., \& Gilfanov, M. 2008, MNRAS, 388, 56

Burwitz, V., Pietsch, W., Updike, A., et al. 2007, The Astronomer's Telegram, 1238,1

Burwitz, V., Henze, M., Pietsch, W., et al. 2008, The Astronomer's Telegram, 1364,1

Capaccioli, M., Della Valle, M., Rosino, L., \& D’Onofrio, M. 1989, AJ, 97, 1622

Catalán, S., Isern, J., García-Berro, E., \& Ribas, I. 2008, MNRAS, 387, 1693

Ciardullo, R., Ford, H., \& Jacoby, G. 1983, ApJ, 272, 92

Ciardullo, R., Ford, H. C., Neill, J. D., Jacoby, G. H., \& Shafter, A. W. 1987, ApJ, 318, 520

Darnley, M. J., Bode, M. F., Kerins, E., et al. 2004, MNRAS, 353, 571

Darnley, M. J., Bode, M. F., Kerins, E., et al. 2006, MNRAS, 369, 257

Della Valle, M. 2002, in Classical Nova Explosions, ed. M. Hernanz, \& J. José, AIP Conf. Ser., 637, 443

Della Valle, M., \& Livio, M. 1995, ApJ, 452, 704

Della Valle, M., \& Livio, M. 1998, ApJ, 506, 818

Della Valle, M., Bianchini, A., Livio, M., \& Orio, M. 1992, A\&A, 266, 232

Della Valle, M., Pasquini, L., Daou, D., \& Williams, R. E. 2002, A\&A, 390, 155

di Mille, F., Ciroi, S., Botte, V., \& Boschetti, C. S. 2003, IAU Circ., 8231, 4 
Dimai, A., \& Manzini, F. 2005, The Astronomer's Telegram, 421, 1

Duerbeck, H. W. 1990, in Physics of Classical Novae, ed. A. Cassatella, \& R. Viotti (Berlin: Springer Verlag), Lect. Not. Phys., 369, IAU Colloq., 122, 34 Fiaschi, M., Di Mille, F., Cariolato, R., Swift, B., \& Li, W. D. 2002, IAU Circ., 7794,1

Fiaschi, M., Tiveron, D., \& Cardullo, A. 2003, IAU Circ., 8226, 2

Filippenko, A. V., \& Chornock, R. 2001, IAU Circ., 7738, 3

Filippenko, A. V., \& Chornock, R. 2002, IAU Circ., 7825, 3

Filippenko, A. V., Chornock, R. T., Coil, A. L., Leonard, D. C., \& Li, W. D. 1999, IAU Circ., 7272, 2

Fruscione, A., McDowell, J. C., Allen, G. E., et al. 2006, SPIE Conf. Ser., 6270

Gabriel, C., Denby, M., Fyfe, D. J., et al. 2004, in Astronomical Data Analysis Software and Systems (ADASS) XIII, ed. F. Ochsenbein, M. G. Allen, \& D. Egret, ASP Conf. Ser., 314, 759

Gallagher, J. S., \& Starrfield, S. 1976, MNRAS, 176, 53

Greiner, J., Hasinger, G., \& Kahabka, P. 1991, A\&A, 246, L17

Hachisu, I., \& Kato, M. 2006, ApJS, 167, 59

Hachisu, I., \& Kato, M. 2010, ApJ, 709, 680

Hachisu, I., Kato, M., \& Luna, G. J. M. 2007, ApJ, 659, L153

Hatano, K., Branch, D., Fisher, A., \& Starrfield, S. 1997, ApJ, 487, L45

Hatzidimitriou, D., Reig, P., Manousakis, A., et al. 2007, A\&A, 464, 1075

Henze, M., Burwitz, V., Pietsch, W., et al. 2007, The Astronomer's Telegram, 1336,1

Henze, M., Burwitz, V., Pietsch, W., et al. 2008a, The Astronomer's Telegram, 1580,1

Henze, M., Meusinger, H., \& Pietsch, W. 2008b, A\&A, 477, 67

Henze, M., Pietsch, W., Burwitz, V., et al. 2008c, The Astronomer's Telegram, 1602,1

Henze, M., Pietsch, W., Haberl, F., et al. 2009a, A\&A, 500, 769

Henze, M., Pietsch, W., Sala, G., et al. 2009b, A\&A, 498, L13

Henze, M., Pietsch, W., Haberl, F., et al. 2010, A\&A, 523, A89 (Paper I)

Hernanz, M. 2005, in The Astrophysics of Cataclysmic Variables and Related Objects, ed. J.-M. Hameury, \& J.-P. Lasota, ASP Conf. Ser., 330, 265

Holland, S. 1998, AJ, 115, 1916

Hornoch, K. 2003, IAU Circ., 8248, 2

Immler, S., Pietsch, W., Kruse, W., et al. 2008, The Astronomer's Telegram, 1673,1

José, J., \& Hernanz, M. 1998, ApJ, 494, 680

Kaaret, P. 2002, ApJ, 578, 114

Kahabka, P., \& van den Heuvel, E. P. J. 1997, ARA\&A, 35, 69

Kahabka, P., Hartmann, H. W., Parmar, A. N., \& Negueruela, I. 1999, A\&A, 347, L43

Kong, A. K. H., Garcia, M. R., Primini, F. A., et al. 2002, ApJ, 577, 738

Krautter, J. 2002, in Classical Nova Explosions, ed. M. Hernanz, \& J. José, AIP Conf. Ser., 637, 345

Lang, F., Lerchster, M., \& Fliri, J. 2006, The Astronomer's Telegram, 821, 1

Livio, M. 1992, ApJ, 393, 516

Metropolis, N., Rosenbluth, A. W., Rosenbluth, M. N., Teller, A. H., \& Teller, E. 1953, J. Chem. Phys., 21, 1087

Nedialkov, P., Orio, M., Birkle, K., et al. 2002, A\&A, 389, 439

Nelson, T., Liu, J. F., di Stefano, R., et al. 2008a, The Astronomer's Telegram, 1672,1

Nelson, T., Orio, M., Cassinelli, J. P., et al. 2008b, ApJ, 673, 1067

Ness, J., Schwarz, G., Starrfield, S., et al. 2008, AJ, 135, 1328

Ness, J.-U., Osborne, J. P., Dobrotka, A., et al. 2011, ApJ, 733, 70

Orio, M. 2006, ApJ, 643, 844

Orio, M., \& Nelson, T. 2008, The Astronomer's Telegram, 1390, 1

Orio, M., Nelson, T., Bianchini, A., Di Mille, F., \& Harbeck, D. 2010, ApJ, 717, 739

Osborne, J., Page, K., Beardmore, A., et al. 2006, The Astronomer's Telegram, 838,1

Ovcharov, E., Valcheva, A., Kostov, A., et al. 2009, The Astronomer's Telegram, 1927,1

Page, K. L., Osborne, J. P., Evans, P. A., et al. 2010, MNRAS, 401, 12

Payne-Gaposchkin, C. 1964, The galactic novae (New York: Dover Publication)

Petz, A., Hauschildt, P. H., Ness, J., \& Starrfield, S. 2005, A\&A, 431, 321

Pietsch, W., Fliri, J., Freyberg, M. J., et al. 2005a, A\&A, 442, 879 (PFF2005)

Pietsch, W., Freyberg, M., \& Haberl, F. 2005b, A\&A, 434, 483
Pietsch, W., Burwitz, V., Greiner, J., et al. 2006a, The Astronomer's Telegram, 850,1

Pietsch, W., Burwitz, V., Rodriguez, J., \& Garcia, A. 2006b, The Astronomer's Telegram, 805, 1

Pietsch, W., Fliri, J., Freyberg, M. J., et al. 2006c, A\&A, 454, 773

Pietsch, W., Burwitz, V., Greiner, J., et al. 2007a, The Astronomer's Telegram, 1009,1

Pietsch, W., Burwitz, V., Updike, A., et al. 2007b, The Astronomer's Telegram, 1257,1

Pietsch, W., Greiner, J., Haberl, F., \& Sala, G. 2007c, The Astronomer's Telegram, 1116, 1

Pietsch, W., Haberl, F., Sala, G., et al. 2007d, A\&A, 465, 375 (PHS2007)

Pietsch, W., Henze, M., Haberl, F., et al. 2011, A\&A, 531, A22

Quimby, R. 2006, The Astronomer's Telegram, 887, 1

Quimby, R., Mondol, P., Hoeflich, P., Wheeler, J. C., \& Gerardy, C. 2005, The Astronomer's Telegram, 600, 1

Rau, A., Burwitz, V., Cenko, S. B., et al. 2007, The Astronomer's Telegram, 1242,1

Rauch, T. 1997, A\&A, 320, 237

Rauch, T. 2003, A\&A, 403, 709

Rauch, T., \& Deetjen, J. L. 2003, in Stellar Atmosphere Modeling, ed. I. Hubeny, D. Mihalas, \& K. Werner, ASP Conf. Ser., 288, 103

Rauch, T., Ringat, E., \& Werner, K. 2010 [arXiv: 1011. 3628]

Rector, T. A., Jacoby, G. H., Corbett, D. L., Denham, M., \& RBSE Nova Search Team 1999, in AAS Meeting Abstracts, 195, 36.08

Ries, C., \& Riffeser, A. 2006, The Astronomer's Telegram, 829, 1

Riffeser, A., Fliri, J., Gössl, C. A., et al. 2001, A\&A, 379, 362

Ritter, H., Politano, M., Livio, M., \& Webbink, R. F. 1991, ApJ, 376, 177

Sala, G., \& Hernanz, M. 2005, A\&A, 439, 1061

Salpeter, E. E. 1955, ApJ, 121, 161

Schaefer, B. E., \& Collazzi, A. C. 2010, AJ, 139, 1831

Schaefer, B. E., Pagnotta, A., Osborne, J. P., et al. 2010, The Astronomer's Telegram, 2477, 1

Schlegel, E. M., Schaefer, B., Pagnotta, A., et al. 2010, The Astronomer's Telegram, 2430, 1

Shafter, A. W. 2007a, The Astronomer's Telegram, 1341, 1

Shafter, A. W. 2007b, The Astronomer's Telegram, 1332, 1

Shafter, A. W., \& Irby, B. K. 2001, ApJ, 563, 749

Shafter, A. W., \& Quimby, R. M. 2007, ApJ, 671, L121

Shafter, A. W., Coelho, E. A., Misselt, K. A., et al. 2006, The Astronomer's Telegram, 923,1

Shanley, L., Ogelman, H., Gallagher, J. S., Orio, M., \& Krautter, J. 1995, ApJ, 438, L95

Sharov, A. S., \& Alksnis, A. 1998, Astron. Lett., 24, 641

Shen, K. J., Idan, I., \& Bildsten, L. 2009, ApJ, 705, 693

Smirnova, O., \& Alksnis, A. 2006, Inform. Bull. Variable Stars, 5720, 1

Smirnova, O., Alksnis, A., \& Zharova, A. V. 2006, Inform. Bull. Variable Stars, 5737,1

Stanek, K. Z., \& Garnavich, P. M. 1998, ApJ, 503, L131

Stark, A. A., Gammie, C. F., Wilson, R. W., et al. 1992, ApJS, 79, 77

Starrfield, S. 1989, in Classical Novae, 39

Starrfield, S., Sparks, W. M., \& Truran, J. W. 1974, ApJS, 28, 247

Stiele, H., Pietsch, W., Haberl, F., \& Freyberg, M. 2008, A\&A, 480, 599

Stiele, H., Pietsch, W., Haberl, F., et al. 2010, Astron. Nachr., 331, 212

Trinchieri, G., \& Fabbiano, G. 1991, ApJ, 382, 82

Trudolyubov, S. P., \& Priedhorsky, W. C. 2008, ApJ, 676, 1218

Truran, J. W., \& Livio, M. 1986, ApJ, 308, 721

Tuchman, Y., \& Truran, J. W. 1998, ApJ, 503, 381

Valcheva, A., Ovcharov, E., Latev, G., et al. 2008, The Astronomer's Telegram, 1687,1

van Rossum, D. R., \& Ness, J. 2010, Astron. Nachr., 331, 175

Voss, R., Pietsch, W., Haberl, F., et al. 2008, A\&A, 489, 707

Walterbos, R. A. M., \& Kennicutt, Jr., R. C. 1988, A\&A, 198, 61

Warner, B. 1995, Cataclysmic variable stars Cambridge Astrophysics Series (Cambridge, New York: Cambridge University Press)

Williams, R. E. 1992, AJ, 104, 725

Williams, B. F., Naik, S., Garcia, M. R., \& Callanan, P. J. 2006, ApJ, 643, 356

Wilms, J., Allen, A., \& McCray, R. 2000, ApJ, 542, 914

Yaron, O., Prialnik, D., Shara, M. M., \& Kovetz, A. 2005, ApJ, 623, 398 
Table 1. Observations of the M 31 monitoring.

\begin{tabular}{|c|c|c|c|c|c|c|c|c|c|}
\hline \multirow[t]{2}{*}{ Telescope/Instrument } & \multirow[t]{2}{*}{ ObsID } & \multicolumn{4}{|c|}{ Exposure time $^{a}[\mathrm{ks}]$} & \multirow{2}{*}{$\begin{array}{c}\text { Start date }^{b} \\
\text { [UT] }\end{array}$} & \multirow{2}{*}{$\begin{array}{c}\mathrm{JD}^{b} \\
2450000+\end{array}$} & \multicolumn{2}{|c|}{ Offset $^{c}$} \\
\hline & & $\mathrm{PN}$ & MOS1 & MOS2 & HRC-I & & & RA ["] & $\operatorname{Dec}\left[{ }^{\prime \prime}\right]$ \\
\hline \multicolumn{10}{|l|}{$2007 / 8$} \\
\hline Chandra HRC-I & 8526 & & & & 19.9 & $2007-11-07.63$ & 4412.14 & -0.3 & 0.3 \\
\hline Chandra HRC-I & 8527 & & & & 20.0 & $2007-11-17.76$ & 4422.26 & -0.3 & 0.3 \\
\hline Chandra HRC-I & 8528 & & & & 20.0 & $2007-11-28.79$ & 4433.29 & -0.2 & 0.2 \\
\hline Chandra HRC-I & 8529 & & & & 18.9 & $2007-12-07.57$ & 4442.07 & -0.3 & 0.1 \\
\hline Chandra HRC-I & 8530 & & & & 19.9 & $2007-12-17.49$ & 4451.99 & -0.3 & 0.1 \\
\hline XMM-Newton EPIC & 0505720201 & 22.3 & 26.9 & 26.9 & & $2007-12-29.57$ & 4464.07 & 0.0 & 0.8 \\
\hline XMM-Newton EPIC & 0505720301 & 20.4 & 26.4 & 26.4 & & 2008-01-08.29 & 4473.79 & 1.3 & 1.4 \\
\hline XMM-Newton EPIC & 0505720401 & 17.2 & 21.2 & 20.9 & & $2008-01-18.63$ & 4484.13 & -3.3 & -0.5 \\
\hline XMM-Newton EPIC & 0505720501 & 9.9 & 15.6 & 14.9 & & $2008-01-27.94$ & 4493.44 & -0.1 & 1.2 \\
\hline XMM-Newton EPIC & 0505720601 & 15.1 & 20.7 & 20.7 & & $2008-02-07.20$ & 4503.75 & 0.2 & 1.0 \\
\hline \multicolumn{10}{|l|}{$2008 / 9$} \\
\hline Chandra HRC-I & 9825 & & & & 20.2 & 2008-11-08.34 & 4778.84 & -0.3 & 0.2 \\
\hline Chandra HRC-I & 9826 & & & & 19.9 & $2008-11-17.14$ & 4787.64 & -0.4 & 0.3 \\
\hline Chandra HRC-I & 9827 & & & & 20.0 & $2008-11-28.24$ & 4798.74 & -0.3 & 0.3 \\
\hline Chandra HRC-I & 9828 & & & & 20.0 & $2008-12-07.41$ & 4807.91 & -0.4 & 0.1 \\
\hline Chandra HRC-I & 9829 & & & & 10.1 & $2008-12-18.02$ & 4818.52 & -0.5 & 0.0 \\
\hline Chandra HRC-I & 10838 & & & & 10.0 & $2008-12-18.49$ & 4818.99 & -0.4 & 0.1 \\
\hline XMM-Newton EPIC & 0551690201 & 15.6 & 21.2 & 21.2 & & $2008-12-30.14$ & 4830.64 & 0.9 & 1.1 \\
\hline XMM-Newton EPIC & 0551690301 & 16.3 & 20.9 & 20.9 & & $2009-01-09.26$ & 4840.76 & 2.1 & 1.0 \\
\hline XMM-Newton EPIC & 0551690401 & 6.1 & 9.9 & 10.0 & & $2009-01-15.90$ & 4847.40 & 0.0 & 1.2 \\
\hline XMM-Newton EPIC & 0551690501 & 13.6 & 19.5 & 18.7 & & $2009-01-27.31$ & 4858.81 & 1.8 & 1.4 \\
\hline XMM-Newton EPIC & 0551690601 & 12.7 & 5.5 & 5.5 & & $2009-02-04.56$ & 4867.06 & -1.7 & -0.6 \\
\hline Chandra HRC-I & 10683 & & & & 19.9 & $2009-02-16.90$ & 4879.40 & -0.5 & 0.1 \\
\hline Chandra HRC-I & 10684 & & & & 18.7 & $2009-02-26.17$ & 4888.67 & -0.5 & 0.1 \\
\hline
\end{tabular}

Notes. ${ }^{(a)}$ Dead-time corrected; for XMM-Newton EPIC after screening for high background. ${ }^{(b)}$ Start time of observations; for XMM-Newton EPIC the PN start time was used. ${ }^{(c)}$ Offset of image WCS (world coordinate system) to the WCS of the catalogue by Kaaret (2002).

Table 2. XMM-Newton and Chandra measurements of M 31 optical nova candidates known from Paper I and still detected here.

\begin{tabular}{|c|c|c|c|c|c|c|c|}
\hline \multicolumn{3}{|c|}{ Optical nova candidate } & \multicolumn{5}{|c|}{$\mathrm{X}$-ray measurements } \\
\hline Name & $\mathrm{RA} \quad(\mathrm{h}: \mathrm{m}: \mathrm{s})^{a}$ & $\mathrm{MJD}^{b}$ & $D^{c}$ & Observation $^{d}$ & $\Delta t^{e}$ & $L_{50}^{f}$ & Comment $^{g}$ \\
\hline $\mathrm{M} 31 \mathrm{~N}$ & $\operatorname{Dec}(\mathrm{d}: \mathrm{m}: \mathrm{s})^{a}$ & (d) & $\left({ }^{\prime \prime}\right)$ & ID & (d) & $\left(10^{36} \mathrm{erg} \mathrm{s}^{-1}\right)$ & \\
\hline \multirow[t]{12}{*}{ 1996-08b } & $0: 42: 55.20$ & 50307.0 & 0.8 & mrg1 (HRC-I) & 4104.6 & $4.1 \pm 0.7$ & SSS \\
\hline & $41: 20: 46.0$ & & 1.2 & 0505720201 (EPIC) & 4156.6 & $2.5 \pm 0.5$ & \\
\hline & & & 1.3 & 0505720301 (EPIC) & 4166.3 & $2.4 \pm 0.4$ & \\
\hline & & & 1.8 & 0505720401 (EPIC) & 4176.6 & $2.5 \pm 0.6$ & \\
\hline & & & 0.5 & 0505720501 (EPIC) & 4185.9 & $2.4 \pm 0.7$ & \\
\hline & & & 2.2 & 0505720601 (EPIC) & 4196.2 & $2.8 \pm 0.6$ & \\
\hline & & & 0.5 & $\operatorname{mrg} 2($ HRC-I) & 4471.3 & $3.9 \pm 0.6$ & \\
\hline & & & 2.3 & 0551690201 (EPIC) & 4523.1 & $2.3 \pm 0.5$ & \\
\hline & & & 2.3 & 0551690301 (EPIC) & 4533.3 & $1.6 \pm 0.5$ & \\
\hline & & & & 0551690401 (EPIC) & 4539.9 & $<4.7$ & \\
\hline & & & & 0551690501 (EPIC) & 4551.3 & $<3.9$ & \\
\hline & & & 1.2 & 0551690601 (EPIC) & 4559.6 & $2.5 \pm 1.3$ & \\
\hline \multirow[t]{6}{*}{ 1997-11a } & $0: 42: 42.13$ & 50753.0 & 0.3 & 8526 (HRC-I) & 3658.6 & $3.5 \pm 0.8$ & SSS-HR \\
\hline & $41: 15: 10.5$ & & 0.4 & 8527 (HRC-I) & 3668.8 & $3.4 \pm 0.8$ & \\
\hline & & & 0.4 & 8528 (HRC-I) & 3679.8 & $2.3 \pm 0.8$ & \\
\hline & & & 0.5 & 8529 (HRC-I) & 3688.6 & $6.0 \pm 1.4$ & \\
\hline & & & 0.5 & 8530 (HRC-I) & 3698.5 & $3.0 \pm 0.9$ & \\
\hline & & & 0.5 & $\operatorname{mrg} 2$ (HRC-I) & 4025.3 & $0.6 \pm 0.2$ & \\
\hline \multirow[t]{9}{*}{ 2001-10a } & $0: 43: 03.31$ & 52186.0 & 1.1 & 8526 (HRC-I) & 2225.6 & $13.3 \pm 2.2$ & SSS \\
\hline & $41: 12: 11.5$ & & 1.0 & 8527 (HRC-I) & 2235.8 & $10.4 \pm 1.9$ & \\
\hline & & & 1.5 & 8528 (HRC-I) & 2246.8 & $9.9 \pm 1.9$ & \\
\hline & & & 1.9 & 8529 (HRC-I) & 2255.6 & $9.8 \pm 1.9$ & \\
\hline & & & & 8530 (HRC-I) & 2265.5 & $<9.8$ & \\
\hline & & & 1.0 & 0505720201 (EPIC) & 2277.6 & $2.6 \pm 0.4$ & \\
\hline & & & & 0505720301 (EPIC) & 2287.3 & $<4.8$ & \\
\hline & & & 0.7 & 0505720401 (EPIC) & 2297.6 & $2.7 \pm 0.6$ & \\
\hline & & & 1.6 & 0505720501 (EPIC) & 2306.9 & $3.4 \pm 0.9$ & \\
\hline
\end{tabular}


M. Henze et al.: X-ray monitoring of classical novae in M 31 in 2007/8 and 2008/9. II.

Table 2. continued.

\begin{tabular}{|c|c|c|c|c|c|c|c|}
\hline \multicolumn{3}{|c|}{ Optical nova candidate } & \multicolumn{5}{|c|}{ X-ray measurements } \\
\hline Name & RA $(\mathrm{h}: \mathrm{m}: \mathrm{s})^{a}$ & $\mathrm{MJD}^{b}$ & $D^{c}$ & Observation $^{d}$ & $\Delta t^{e}$ & $L_{50}^{f}$ & Comment $^{g}$ \\
\hline M31N & $\operatorname{Dec}(\mathrm{d}: \mathrm{m}: \mathrm{s})^{a}$ & (d) & $\left({ }^{\prime \prime}\right)$ & ID & (d) & $\left(10^{36} \mathrm{erg} \mathrm{s}^{-1}\right)$ & \\
\hline & & & 1.8 & 0505720601 (EPIC) & 2317.2 & $3.6 \pm 0.7$ & \\
\hline & & & 1.5 & 9825 (HRC-I) & 2592.3 & $7.0 \pm 1.8$ & \\
\hline & & & 2.1 & 9826 (HRC-I) & 2601.1 & $8.1 \pm 1.7$ & \\
\hline & & & 0.6 & 9827 (HRC-I) & 2612.2 & $11.3 \pm 2.2$ & \\
\hline & & & 0.3 & 9828 (HRC-I) & 2621.4 & $10.8 \pm 2.1$ & \\
\hline & & & 0.9 & 9829 (HRC-I) & 2632.0 & $11.9 \pm 3.4$ & \\
\hline & & & & 10838 (HRC-I) & 2632.5 & $<20.4$ & \\
\hline & & & 0.7 & 0551690201 (EPIC) & 2644.1 & $3.6 \pm 0.6$ & \\
\hline & & & 1.7 & 0551690301 (EPIC) & 2654.3 & $3.7 \pm 0.7$ & \\
\hline & & & & 0551690401 (EPIC) & 2660.9 & $<6.1$ & \\
\hline & & & & 0551690501 (EPIC) & 2672.3 & $<4.0$ & \\
\hline & & & 1.1 & 0551690601 (EPIC) & 2680.6 & $3.4 \pm 0.9$ & \\
\hline & & & 1.4 & 10683 (HRC-I) & 2692.9 & $7.3 \pm 1.7$ & \\
\hline & & & & 10684 (HRC-I) & 2702.2 & $<11.8$ & \\
\hline \multirow[t]{23}{*}{$2004-05 b$} & $0: 42: 37.04$ & 53143.0 & 0.4 & 8526 (HRC-I) & 1268.6 & $20.9 \pm 2.3$ & SSS \\
\hline & $41: 14: 28.5$ & & 0.4 & 8527 (HRC-I) & 1278.8 & $47.9 \pm 6.0$ & \\
\hline & & & 0.3 & 8528 (HRC-I) & 1289.8 & $21.5 \pm 2.6$ & \\
\hline & & & 0.3 & 8529 (HRC-I) & 1298.6 & $24.8 \pm 2.9$ & \\
\hline & & & 0.3 & 8530 (HRC-I) & 1308.5 & $31.4 \pm 3.4$ & \\
\hline & & & 1.4 & 0505720201 (EPIC) & 1320.6 & $21.9 \pm 0.9$ & \\
\hline & & & 1.2 & 0505720301 (EPIC) & 1330.3 & $20.0 \pm 0.9$ & \\
\hline & & & 1.1 & 0505720401 (EPIC) & 1340.6 & $21.6 \pm 1.0$ & \\
\hline & & & 1.2 & 0505720501 (EPIC) & 1349.9 & $21.4 \pm 1.3$ & \\
\hline & & & 1.0 & 0505720601 (EPIC) & 1360.2 & $16.7 \pm 1.0$ & \\
\hline & & & 0.3 & 9825 (HRC-I) & 1635.3 & $19.5 \pm 2.2$ & \\
\hline & & & 0.4 & 9826 (HRC-I) & 1644.1 & $17.9 \pm 2.2$ & \\
\hline & & & 0.4 & 9827 (HRC-I) & 1655.2 & $25.4 \pm 2.8$ & \\
\hline & & & 0.4 & 9828 (HRC-I) & 1664.4 & $21.3 \pm 2.6$ & \\
\hline & & & 0.4 & 9829 (HRC-I) & 1675.0 & $19.9 \pm 3.0$ & \\
\hline & & & 0.4 & 10838 (HRC-I) & 1675.5 & $26.6 \pm 3.9$ & \\
\hline & & & 1.2 & 0551690201 (EPIC) & 1687.1 & $18.4 \pm 1.0$ & \\
\hline & & & 0.8 & 0551690301 (EPIC) & 1697.3 & $16.5 \pm 1.0$ & \\
\hline & & & 0.6 & 0551690401 (EPIC) & 1703.9 & $16.7 \pm 1.9$ & \\
\hline & & & 1.2 & 0551690501 (EPIC) & 1715.3 & $17.0 \pm 1.0$ & \\
\hline & & & 0.6 & 0551690601 (EPIC) & 1723.6 & $16.7 \pm 1.2$ & \\
\hline & & & 0.7 & 10683 (HRC-I) & 1735.9 & $33.9 \pm 4.2$ & \\
\hline & & & 0.5 & 10684 (HRC-I) & 1745.2 & $36.7 \pm 4.6$ & \\
\hline
\end{tabular}

Notes. ${ }^{(a)}$ RA, Dec are given in J2000.0; ${ }^{(b)}$ modified Julian Date of optical outburst; MJD = JD - 2400000.5 ; ${ }^{(c)}$ distance in arcsec between optical and X-ray source; ${ }^{(d)} \mathrm{mrg} 1 / 2$ (HRC-I/EPIC) indicates merged data of all HRC-I/EPIC observations during 2007/8 or 2008/9, mrg3 (HRC-I) indicates merged data of the Chandra observations 9829 and 10838 (taken on the same day); ${ }^{(e)}$ time after observed start of optical outburst; (f) unabsorbed equivalent luminosity in the $0.2-10.0 \mathrm{keV}$ band assuming a $50 \mathrm{eV}$ blackbody spectrum with Galactic foreground absorption (luminosity errors are $1 \sigma$, upper limits are $3 \sigma) ;{ }^{(g)}$ SSS or SSS-HR indicate X-ray sources classified as supersoft based on XMM-Newton spectra or Chandra hardness ratios, respectively. 
A\&A 533, A52 (2011)

Table 3. Best-fit spectral parameters for nova SSSs for blackbody (BB) and NLTE WD atmosphere models with solar (WD S) and halo (WD H) abundances.

\begin{tabular}{|c|c|c|c|c|c|}
\hline $\begin{array}{l}\text { Name } \\
\text { M31N }\end{array}$ & Model & $\begin{array}{c}k T \\
(\mathrm{eV})\end{array}$ & $\begin{array}{c}N_{\mathrm{H}} \\
\left(10^{21} \mathrm{~cm}^{-2}\right)\end{array}$ & $\chi^{2} /$ d.o.f. & d.o.f. \\
\hline \multirow[t]{3}{*}{ 1996-08b } & $\mathrm{BB}$ & $21.0_{-13.0}^{+8.0}$ & $1.4_{-0.8}^{+1.2}$ & 1.58 & \multirow[t]{3}{*}{29} \\
\hline & WD S & $44.0_{-6.0}^{+1.0}$ & $0.3_{-0.2}^{+0.3}$ & 1.61 & \\
\hline & WD H & $35.0_{-9.0}^{+3.0}$ & $0.4_{-0.3}^{+0.3}$ & 1.63 & \\
\hline \multirow[t]{3}{*}{ 2001-10a } & $\mathrm{BB}$ & $14.0_{-7.0}^{+4.0}$ & $2.3_{-0.3}^{+0.3}$ & 1.43 & \multirow[t]{3}{*}{27} \\
\hline & WD S & $26.0_{-4.0}^{+17.0}$ & $0.7_{-0.2}^{+0.5}$ & 1.15 & \\
\hline & WD H & $34.0_{-9.0}^{+1.0}$ & $0.2_{-0.1}^{+0.1}$ & 1.22 & \\
\hline \multirow[t]{3}{*}{$2004-05 b$} & $\mathrm{BB}$ & $30.0_{-5.0}^{+6.0}$ & $1.5_{-0.4}^{+0.5}$ & 1.19 & \multirow[t]{3}{*}{44} \\
\hline & WD S & $45.0_{-1.0}^{+2.0}$ & $1.0_{-0.3}^{+0.8}$ & 1.32 & \\
\hline & WD H & $38.0_{-3.0}^{+7.0}$ & $1.1_{-0.5}^{+0.7}$ & 1.30 & \\
\hline \multirow[t]{3}{*}{$2005-02 a$} & $\mathrm{BB}$ & $38.0_{-8.0}^{+6.0}$ & $0.5_{-0.2}^{+0.4}$ & 1.20 & \multirow[t]{3}{*}{26} \\
\hline & WD S & $46.0_{-2.0}^{+6.0}$ & $0.2_{-0.2}^{+0.2}$ & 1.15 & \\
\hline & WD H & $44.0_{-1.0}^{+2.0}$ & $0.2_{-0.1}^{+0.1}$ & 1.20 & \\
\hline \multirow[t]{3}{*}{ 2006-04a } & $\mathrm{BB}$ & $54.0_{-10.0}^{+9.0}$ & $1.3_{-0.5}^{+0.8}$ & 0.49 & \multirow[t]{3}{*}{15} \\
\hline & WD S & $66.0_{-3.0}^{+3.0}$ & $0.7_{-0.3}^{+0.4}$ & 0.56 & \\
\hline & WD H & $58.0_{-3.0}^{+3.0}$ & $0.8_{-0.3}^{+0.4}$ & 0.58 & \\
\hline \multirow[t]{3}{*}{$2006-06 b$} & $\mathrm{BB}$ & $37.0_{-150}^{+17.0}$ & $0.5_{-0.2}^{+0.9}$ & 0.99 & \multirow[t]{3}{*}{12} \\
\hline & WD S & $46.0_{-2.0}^{+7.0}$ & $0.2_{-0.2}^{+0.4}$ & 1.07 & \\
\hline & WD H & $44.0_{-8.0}^{+3.0}$ & $0.1_{-0.1}^{+0.5}$ & 1.06 & \\
\hline \multirow[t]{3}{*}{$2006-09 c$} & $\mathrm{BB}$ & $74.0_{-24.0}^{+20.0}$ & $0.2_{-0.2}^{+0.8}$ & 0.97 & \multirow[t]{3}{*}{4} \\
\hline & WD S & $71.0_{-8.0}^{+5.0}$ & $0.1_{-0.1}^{+0.3}$ & 0.96 & \\
\hline & WD H & $63.0_{-9.0}^{+7.0}$ & $0.1_{-0.1}^{+0.5}$ & 0.98 & \\
\hline \multirow[t]{3}{*}{$2007-02 b$} & $\mathrm{BB}$ & $28.0_{-10.0}^{+11.0}$ & $2.5_{-1.1}^{+1.8}$ & 0.96 & \multirow[t]{3}{*}{13} \\
\hline & WD S & $43.0_{-7.0}^{+3.0}$ & $3.2_{-1.8}^{+0.7}$ & 0.77 & \\
\hline & WD H & $35.0_{-1.0}^{+10.0}$ & $2.7_{-1.7}^{+0.8}$ & 0.77 & \\
\hline \multirow[t]{3}{*}{$2007-06 b^{*}$} & $\mathrm{BB}$ & $48.0_{-3.0}^{+2.0}$ & $2.3_{-0.1}^{+0.1}$ & 1.39 & \multirow[t]{3}{*}{53} \\
\hline & WD S & $70.0_{-1.0}^{+1.0}$ & $1.0_{-0.2}^{+0.2}$ & 1.01 & \\
\hline & WD H & $61.0_{-1.0}^{+1.0}$ & $1.0_{-0.2}^{+0.2}$ & 1.06 & \\
\hline \multirow[t]{3}{*}{$2007-10 b$} & BB & $66.0_{-24.0}^{+34.0}$ & $0.9_{-0.8}^{+1.5}$ & 0.4 & \multirow[t]{3}{*}{2} \\
\hline & WD S & $70.0_{-8.0}^{+9.0}$ & $0.5_{-0.5}^{+1.2}$ & 0.4 & \\
\hline & WD H & $61.0_{-9.0}^{+10.0}$ & $0.5_{-0.5}^{+1.3}$ & 0.4 & \\
\hline \multirow[t]{3}{*}{$2007-12 b^{* *}$} & $\mathrm{BB}$ & $81.0_{-2.0}^{+2.0}$ & $1.8_{-0.2}^{+0.2}$ & 2.68 & \multirow[t]{3}{*}{112} \\
\hline & WD S & $80.0_{-1.0}^{+1.0}$ & $0.9_{-0.1}^{+0.1}$ & 1.06 & \\
\hline & WD H & $76.0_{-1.0}^{+1.0}$ & $0.8_{-0.1}^{+0.1}$ & 0.98 & \\
\hline \multirow[t]{3}{*}{$2007-12 d$} & BB & $66.0_{-28.0}^{+72.0}$ & $0.9_{-0.9}^{+9.9}$ & 0.4 & \multirow[t]{3}{*}{2} \\
\hline & WD S & $69.0_{-16.0}^{+10.0}$ & $0.7_{-0.7}^{+2.1}$ & 0.4 & \\
\hline & WD H & $61.0_{-16.0}^{+11.0}$ & $0.8_{-0.8}^{+2.0}$ & 0.4 & \\
\hline \multirow[t]{3}{*}{$2008-05 a$} & BB & $45.0_{-28.0}^{+25.0}$ & $0.4_{-0.4}^{+1.8}$ & 1.00 & \multirow[t]{3}{*}{8} \\
\hline & WD S & $45.0_{-2.0}^{+10.0}$ & $0.5_{-0.5}^{+0.5}$ & 1.62 & \\
\hline & WD H & $39.0_{-3.0}^{+12.0}$ & $0.4_{-0.4}^{+0.9}$ & 1.65 & \\
\hline
\end{tabular}

Notes. ${ }^{(*)}$ From Henze et al. (2009a); ${ }^{(*)}$ from Pietsch et al. (2011). 
M. Henze et al.: X-ray monitoring of classical novae in M 31 in 2007/8 and 2008/9. II.

Table 4. M 31 optical novae with XMM-Newton and Chandra counterparts discovered in this work.

\begin{tabular}{|c|c|c|c|c|c|c|c|}
\hline \multicolumn{3}{|c|}{ Optical nova candidate } & \multicolumn{5}{|c|}{ X-ray measurements } \\
\hline Name & RA $(\mathrm{h}: \mathrm{m}: \mathrm{s})^{a}$ & $\mathrm{MJD}^{b}$ & $D^{c}$ & Observation $^{d}$ & $\Delta t^{e}$ & $L_{50}^{f}$ & Comment $^{g}$ \\
\hline M31N & $\operatorname{Dec}(\mathrm{d}: \mathrm{m}: \mathrm{s})^{a}$ & (d) & $\left({ }^{\prime \prime}\right)$ & ID & (d) & $\left(10^{36} \mathrm{erg} \mathrm{s}^{-1}\right)$ & \\
\hline \multirow{15}{*}{$2003-08 \mathrm{c}$} & $0: 42: 41.20$ & 52878.0 & & 8526 (HRC-I) & 1533.6 & $<2.1$ & \\
\hline & $41: 16: 16.0$ & & 0.4 & 8527 (HRC-I) & 1543.8 & $3.5 \pm 0.7$ & \\
\hline & & & & 8528 (HRC-I) & 1554.8 & $<3.3$ & \\
\hline & & & & 8529 (HRC-I) & 1563.6 & $2.6 \pm 0.8$ & \\
\hline & & & 0.3 & 8530 (HRC-I) & 1573.5 & $3.6 \pm 0.8$ & \\
\hline & & & & mrg1 (EPIC) & 1585.6 & $<11.3$ & \\
\hline & & & & 9825 (HRC-I) & 1900.3 & $<0.8$ & \\
\hline & & & 0.5 & 9826 (HRC-I) & 1909.1 & $5.3 \pm 1.1$ & \\
\hline & & & 0.3 & 9827 (HRC-I) & 1920.2 & $4.7 \pm 1.0$ & \\
\hline & & & 0.3 & 9828 (HRC-I) & 1929.4 & $5.5 \pm 1.1$ & \\
\hline & & & & 9829 (HRC-I) & 1940.0 & $<6.2$ & \\
\hline & & & & 10838 (HRC-I) & 1940.5 & $<5.0$ & \\
\hline & & & & mrg2 (EPIC) & 1952.1 & $<7.0$ & \\
\hline & & & & 10683 (HRC-I) & 2000.9 & $<3.2$ & \\
\hline & & & 0.2 & 10684 (HRC-I) & 2010.2 & $2.7 \pm 0.8$ & \\
\hline \multirow[t]{10}{*}{ 2004-01b } & $0: 42: 41.19$ & 53005.8 & & 8530 (HRC-I) & 1445.7 & $<1.6$ & \\
\hline & $41: 15: 45.0$ & & 0.4 & 9825 (HRC-I) & 1772.6 & $5.0 \pm 1.1$ & \\
\hline & & & 0.2 & 9826 (HRC-I) & 1781.4 & $5.9 \pm 1.2$ & \\
\hline & & & 0.4 & 9827 (HRC-I) & 1792.5 & $5.1 \pm 1.1$ & \\
\hline & & & 0.3 & 9828 (HRC-I) & 1801.7 & $5.8 \pm 1.3$ & \\
\hline & & & 0.4 & 9829 (HRC-I) & 1812.3 & $5.7 \pm 1.3$ & \\
\hline & & & 0.2 & 10838 (HRC-I) & 1812.7 & $7.1 \pm 1.5$ & \\
\hline & & & & mrg2 (EPIC) & 1824.4 & $<22.3$ & \\
\hline & & & 0.1 & 10683 (HRC-I) & 1873.1 & $11.1 \pm 1.6$ & \\
\hline & & & 0.1 & 10684 (HRC-I) & 1882.4 & $8.7 \pm 1.7$ & \\
\hline \multirow[t]{14}{*}{$2006-06 b$} & $0: 42: 32.77$ & 53869.0 & & 0505720601 (EPIC) & 634.2 & $<1.8$ & SSS \\
\hline & $41: 16: 49.2$ & & 0.4 & 9825 (HRC-I) & 909.3 & $3.3 \pm 0.7$ & \\
\hline & & & & 9826 (HRC-I) & 918.1 & $<2.6$ & \\
\hline & & & 0.5 & 9827 (HRC-I) & 929.2 & $1.9 \pm 0.6$ & \\
\hline & & & 0.3 & 9828 (HRC-I) & 938.4 & $2.8 \pm 0.6$ & \\
\hline & & & 0.1 & 9829 (HRC-I) & 949.0 & $3.3 \pm 1.2$ & \\
\hline & & & & 10838 (HRC-I) & 949.5 & $<3.3$ & \\
\hline & & & 0.9 & 0551690201 (EPIC) & 961.1 & $3.3 \pm 0.5$ & \\
\hline & & & 0.3 & 0551690301 (EPIC) & 971.3 & $2.9 \pm 0.5$ & \\
\hline & & & & 0551690401 (EPIC) & 977.9 & $<5.8$ & \\
\hline & & & 0.3 & 0551690501 (EPIC) & 989.3 & $4.4 \pm 0.6$ & \\
\hline & & & 0.8 & 0551690601 (EPIC) & 997.6 & $4.0 \pm 0.9$ & \\
\hline & & & 0.1 & 10683 (HRC-I) & 1009.9 & $10.9 \pm 1.7$ & \\
\hline & & & 0.2 & 10684 (HRC-I) & 1019.2 & $7.2 \pm 1.4$ & \\
\hline \multirow[t]{11}{*}{$2006-09 \mathrm{c}$} & $0: 42: 42.38$ & 53996.2 & & 8526 (HRC-I) & 415.4 & $<4.5$ & SSS \\
\hline & $41: 08: 45.5$ & & & 8527 (HRC-I) & 425.5 & $4.2 \pm 1.6$ & \\
\hline & & & & 8528 (HRC-I) & 436.5 & $5.1 \pm 2.0$ & \\
\hline & & & & 8529 (HRC-I) & 445.3 & $<5.3$ & \\
\hline & & & & 8530 (HRC-I) & 455.2 & $<7.2$ & \\
\hline & & & 1.2 & 0505720201 (EPIC) & 467.3 & $2.5 \pm 0.6$ & \\
\hline & & & 0.5 & 0505720301 (EPIC) & 477.0 & $1.6 \pm 0.6$ & \\
\hline & & & 1.0 & 0505720401 (EPIC) & 487.4 & $2.8 \pm 0.8$ & \\
\hline & & & & 0505720501 (EPIC) & 496.7 & $<2.7$ & \\
\hline & & & & 0505720601 (EPIC) & 507.0 & $<1.9$ & \\
\hline & & & & mrg2 (EPIC) & 833.9 & $<0.3$ & \\
\hline \multirow[t]{8}{*}{$2007-02 b$} & $0: 41: 40.32$ & 54134.8 & 1.0 & 0505720401 (EPIC) & 348.9 & $1.8 \pm 0.7$ & SSS \\
\hline & $41: 14: 33.5$ & & & 0505720501 (EPIC) & 358.2 & $<5.6$ & \\
\hline & & & 0.5 & 0505720601 (EPIC) & 368.5 & $6.2 \pm 1.1$ & \\
\hline & & & 0.7 & 0551690201 (EPIC) & 695.4 & $17.4 \pm 5.3$ & \\
\hline & & & 1.3 & 0551690301 (EPIC) & 705.5 & $17.5 \pm 5.4$ & \\
\hline & & & 3.0 & 0551690401 (EPIC) & 712.1 & $9.7 \pm 3.0$ & \\
\hline & & & 0.2 & 0551690501 (EPIC) & 723.6 & $9.7 \pm 1.2$ & \\
\hline & & & 1.2 & 0551690601 (EPIC) & 731.8 & $10.5 \pm 1.8$ & \\
\hline
\end{tabular}


Table 4. continued.

\begin{tabular}{|c|c|c|c|c|c|c|c|}
\hline \multicolumn{3}{|c|}{ Optical nova candidate } & \multicolumn{5}{|c|}{$\mathrm{X}$-ray measurements } \\
\hline Name & RA $\quad(\mathrm{h}: \mathrm{m}: \mathrm{s})^{a}$ & $\mathrm{MJD}^{b}$ & $D^{c}$ & Observation $^{d}$ & $\Delta t^{e}$ & $\begin{array}{c}L_{50}^{f} \\
\left(10^{36} \mathrm{ero} \mathrm{s}^{-1}\right)\end{array}$ & Comment $^{g}$ \\
\hline $2007-06 b$ & $\begin{array}{l}0: 42: 33.14 \\
41: 00: 25.9\end{array}$ & 54270.0 & $\begin{array}{l}9.4 \\
9.4 \\
1.2 \\
9.7 \\
0.6 \\
5.9\end{array}$ & $\begin{array}{l}8526 \text { (HRC-I) } \\
8527 \text { (HRC-I) } \\
8528 \text { (HRC-I) } \\
8529 \text { (HRC-I) } \\
8530 \text { (HRC-I) } \\
\text { mrg2 (HRC-I) }\end{array}$ & $\begin{array}{l}141.6 \\
151.8 \\
162.8 \\
171.6 \\
181.5 \\
508.3\end{array}$ & $\begin{array}{r}73.6 \pm 16.6 \\
72.4 \pm 9.9 \\
80.9 \pm 10.5 \\
88.0 \pm 11.5 \\
96.7 \pm 12.0 \\
<14.5\end{array}$ & $\begin{array}{l}\text { SSS } \\
\text { see (1) }\end{array}$ \\
\hline $2007-10 b$ & $\begin{array}{l}0: 43: 29.48 \\
41: 17: 13.5\end{array}$ & 54386.2 & $\begin{array}{l}1.2 \\
2.4 \\
2.5\end{array}$ & $\begin{array}{r}8526 \text { (HRC-I) } \\
8527 \text { (HRC-I) } \\
8528 \text { (HRC-I) } \\
8529 \text { (HRC-I) } \\
8530 \text { (HRC-I) } \\
0505720201 \text { (EPIC) } \\
0505720301 \text { (EPIC) } \\
0505720401 \text { (EPIC) } \\
0505720501 \text { (EPIC) } \\
0505720601 \text { (EPIC) }\end{array}$ & $\begin{array}{r}25.4 \\
35.5 \\
46.5 \\
55.3 \\
65.2 \\
77.3 \\
87.0 \\
97.4 \\
106.7 \\
117.0\end{array}$ & $\begin{array}{r}31.5 \pm 4.4 \\
24.2 \pm 4.0 \\
16.7 \pm 3.4 \\
<10.1 \\
8.4 \pm 3.0 \\
5.0 \pm 0.7 \\
3.5 \pm 0.7 \\
<2.3 \\
<2.1 \\
<0.6\end{array}$ & SSS \\
\hline 2007-11a & $\begin{array}{l}0: 42: 37.29 \\
41: 17: 10.3\end{array}$ & 54406.2 & $\begin{array}{l}0.4 \\
0.3 \\
0.2 \\
0.2\end{array}$ & $\begin{array}{r}8526 \text { (HRC-I) } \\
8527 \text { (HRC-I) } \\
8528 \text { (HRC-I) } \\
8529 \text { (HRC-I) } \\
8530 \text { (HRC-I) } \\
0505720201 \text { (EPIC) } \\
0505720301 \text { (EPIC) } \\
0505720401 \text { (EPIC) } \\
0505720501 \text { (EPIC) } \\
0505720601 \text { (EPIC) }\end{array}$ & $\begin{array}{r}5.4 \\
15.5 \\
26.5 \\
35.3 \\
45.2 \\
57.3 \\
67.0 \\
77.4 \\
86.7 \\
97.0\end{array}$ & $\begin{aligned} & <1.3 \\
65.5 & \pm 4.3 \\
16.1 & \pm 1.8 \\
17.7 & \pm 1.9 \\
11.2 & \pm 1.6 \\
& <1.1 \\
& <2.3 \\
& <2.6 \\
& <1.9 \\
& <3.2\end{aligned}$ & $\begin{array}{l}\text { SSS-HR } \\
\text { see (2) }\end{array}$ \\
\hline $2007-12 b$ & $\begin{array}{l}0: 43: 19.94 \\
41: 13: 46.6\end{array}$ & 54443.5 & $\begin{array}{l}0.8 \\
0.8 \\
0.7 \\
0.6\end{array}$ & $\begin{array}{r}8530 \text { (HRC-I) } \\
0505720201 \text { (EPIC) } \\
0505720301 \text { (EPIC) } \\
0505720401 \text { (EPIC) } \\
0505720501 \text { (EPIC) } \\
0505720601 \text { (EPIC) } \\
\text { mrg2 (HRC-I) } \\
\text { mrg2 (EPIC) }\end{array}$ & $\begin{array}{r}8.0 \\
20.1 \\
29.8 \\
40.1 \\
49.4 \\
59.8 \\
334.8 \\
386.6\end{array}$ & $\begin{aligned} & <3.5 \\
& <2.1 \\
241.1 & \pm 3.1 \\
335.8 & \pm 3.8 \\
326.0 & \pm 5.2 \\
222.5 & \pm 3.8 \\
& <1.3 \\
& <0.2\end{aligned}$ & $\begin{array}{l}\text { SSS } \\
\text { see (3) }\end{array}$ \\
\hline $2007-12 d$ & $\begin{array}{l}0: 41: 54.96 \\
41: 09: 47.3\end{array}$ & 54451.5 & 0.9 & $\begin{array}{l}0505720201 \text { (EPIC) } \\
0505720301 \text { (EPIC) } \\
0505720401 \text { (EPIC) } \\
0505720501 \text { (EPIC) } \\
0505720601 \text { (EPIC) }\end{array}$ & $\begin{array}{l}12.1 \\
21.8 \\
32.1 \\
41.4 \\
51.8\end{array}$ & $\begin{array}{r}<1.3 \\
2.8 \pm 0.8 \\
<1.6 \\
<0.5 \\
<1.3\end{array}$ & SSS-HR \\
\hline $2008-05 a$ & $\begin{array}{l}0: 42: 56.84 \\
41: 11: 52.4\end{array}$ & 54600.8 & $\begin{array}{l}0.6 \\
1.1 \\
0.4\end{array}$ & $\begin{array}{r}9825 \text { (HRC-I) } \\
9826 \text { (HRC-I) } \\
9827 \text { (HRC-I) } \\
9828 \text { (HRC-I) } \\
9829 \text { (HRC-I) } \\
10838 \text { (HRC-I) } \\
0551690201 \text { (EPIC) } \\
0551690301 \text { (EPIC) } \\
0551690401 \text { (EPIC) } \\
0551690501 \text { (EPIC) } \\
0551690601 \text { (EPIC) } \\
10683 \text { (HRC-I) } \\
10684 \text { (HRC-I) }\end{array}$ & $\begin{array}{l}177.6 \\
186.4 \\
197.5 \\
206.7 \\
217.3 \\
217.7 \\
229.4 \\
239.5 \\
246.1 \\
257.6 \\
265.8 \\
278.1 \\
287.4\end{array}$ & $\begin{aligned} &<4.4 \\
&<4.9 \\
& 7.3 \pm 1.8 \\
& 3.5 \pm 1.5 \\
&<5.7 \\
& 5.8 \pm 2.5 \\
&<1.9 \\
&<3.3 \\
&<4.2 \\
& 3.5 \pm 0.6 \\
& 8.3 \pm 1.3 \\
& 11.1 \pm 1.9 \\
& 5.3 \pm 1.8\end{aligned}$ & SSS \\
\hline
\end{tabular}


M. Henze et al.: X-ray monitoring of classical novae in M 31 in 2007/8 and 2008/9. II.

Table 4. continued.

\begin{tabular}{|c|c|c|c|c|c|c|c|}
\hline \multicolumn{3}{|c|}{ Optical nova candidate } & \multicolumn{5}{|c|}{ X-ray measurements } \\
\hline Name & RA $(\mathrm{h}: \mathrm{m}: \mathrm{s})^{a}$ & $\mathrm{MJD}^{b}$ & $D^{c}$ & Observation $^{d}$ & $\Delta t^{e}$ & $L_{50}^{f}$ & Comment $^{g}$ \\
\hline M31N & $\operatorname{Dec}(\mathrm{d}: \mathrm{m}: \mathrm{s})^{a}$ & (d) & $\left({ }^{\prime \prime}\right)$ & ID & (d) & $\left(10^{36} \mathrm{erg} \mathrm{s}^{-1}\right)$ & \\
\hline \multirow{8}{*}{$2008-05 b$} & $0: 42: 52.88$ & 54608.8 & & 9825 (HRC-I) & 169.6 & $<5.1$ & \\
\hline & $41: 16: 39.4$ & & & 9826 (HRC-I) & 178.4 & $<5.5$ & \\
\hline & & & 1.4 & 9827 (HRC-I) & 189.5 & $3.5 \pm 0.9$ & \\
\hline & & & 1.0 & 9828 (HRC-I) & 198.7 & $4.9 \pm 1.3$ & \\
\hline & & & & mrg3 (HRC-I) & 209.3 & $3.6 \pm 1.1$ & \\
\hline & & & & mrg2 (EPIC) & 221.4 & $<3.2$ & \\
\hline & & & & 10683 (HRC-I) & 270.1 & $<1.7$ & \\
\hline & & & & 10684 (HRC-I) & 279.4 & $<2.3$ & \\
\hline \multirow[t]{13}{*}{ 2008-06a } & $0: 42: 37.72$ & 54631.5 & & 9825 (HRC-I) & 146.8 & $<1.1$ & \\
\hline & $41: 12: 30.0$ & & & 9826 (HRC-I) & 155.6 & $<2.7$ & \\
\hline & & & & 9827 (HRC-I) & 166.7 & $<1.0$ & \\
\hline & & & & 9828 (HRC-I) & 175.9 & $<2.2$ & \\
\hline & & & & 9829 (HRC-I) & 186.5 & $<1.9$ & \\
\hline & & & & 10838 (HRC-I) & 187.0 & $<2.8$ & \\
\hline & & & & 0551690201 (EPIC) & 198.6 & $<0.2$ & \\
\hline & & & & 0551690301 (EPIC) & 208.8 & $<3.3$ & \\
\hline & & & & 0551690401 (EPIC) & 215.4 & $<1.6$ & \\
\hline & & & & 0551690501 (EPIC) & 226.8 & $<1.6$ & \\
\hline & & & & 0551690601 (EPIC) & 235.1 & $<2.3$ & \\
\hline & & & & 10683 (HRC-I) & 247.4 & $<4.9$ & \\
\hline & & & 0.7 & 10684 (HRC-I) & 256.7 & $3.2 \pm 1.3$ & \\
\hline
\end{tabular}

Notes. As for Table 2. Additional comments refer to individual sources discussed in detail in the following papers: (1): Henze et al. (2009a), (2): Henze et al. (2009b), (3): Pietsch et al. (2011).

Table 5. Upper limits for non-detected M 31 CNe from Paper I.

\begin{tabular}{|c|c|c|c|c|c|c|}
\hline \multicolumn{3}{|c|}{ Optical nova candidate } & \multicolumn{3}{|c|}{ X-ray measurements } & \multirow[b]{2}{*}{ Comment } \\
\hline $\begin{array}{l}\text { Name } \\
\text { M31N }\end{array}$ & $\begin{array}{l}\mathrm{RA}(\mathrm{h}: \mathrm{m}: \mathrm{s})^{a} \\
\operatorname{Dec}(\mathrm{d}: \mathrm{m}: \mathrm{s})^{a}\end{array}$ & $\begin{array}{l}\mathrm{MJD}^{b} \\
\text { (d) }\end{array}$ & $\begin{array}{l}\text { Observation }^{d} \\
\text { ID }\end{array}$ & $\begin{array}{l}\Delta t^{e} \\
(\mathrm{~d})\end{array}$ & $\begin{array}{c}L_{50}{ }^{f} \\
\left(10^{36} \mathrm{erg} \mathrm{s}^{-1}\right)\end{array}$ & \\
\hline $2001-01 a$ & $\begin{array}{l}0: 42: 21.49 \\
41: 07: 47.4\end{array}$ & 51928.8 & $\begin{array}{l}\text { mrg1 (HRC-I) } \\
\text { mrg1 (EPIC) }\end{array}$ & $\begin{array}{l}2482.9 \\
2534.8\end{array}$ & $\begin{array}{l}<1.5 \\
<0.6\end{array}$ & $\begin{array}{l}\text { very faint } \\
\text { in Paper I }\end{array}$ \\
\hline $2005-02 a$ & $\begin{array}{l}0: 42: 52.79 \\
41: 14: 28.9\end{array}$ & 53419.8 & $\begin{array}{l}\text { mrg1 (HRC-I) } \\
\text { mrg1 (EPIC) }\end{array}$ & $\begin{array}{r}991.9 \\
1043.8\end{array}$ & $\begin{array}{l}<0.5 \\
<1.8\end{array}$ & \\
\hline
\end{tabular}

Notes. As for Table 2. 
Table 6. Upper limits for M $31 \mathrm{CNe}$ with outburst from about one year before the start of the 2007/8 monitoring till its end.

\begin{tabular}{|c|c|c|c|c|c|c|}
\hline $\begin{array}{l}\text { Optical no } \\
\text { Name } \\
\text { M31N }\end{array}$ & $\begin{array}{l}\text { candidate } \\
\text { RA (h:m:s })^{a} \\
\text { Dec }(\mathrm{d}: \mathrm{m}: \mathrm{s})^{a}\end{array}$ & $\begin{array}{l}\mathrm{MJD}^{b} \\
\text { (d) }\end{array}$ & $\begin{array}{l}\text { X-ray measurements } \\
\text { Observation }^{d} \\
\text { ID }\end{array}$ & $\begin{array}{l}\Delta t^{e} \\
(\mathrm{~d})\end{array}$ & $\begin{array}{c}L_{50}{ }^{f} \\
\left(10^{36} \mathrm{erg} \mathrm{s}^{-1}\right)\end{array}$ & Comment \\
\hline $2006-10 a$ & $\begin{array}{l}0: 41: 43.23 \\
41: 11: 45.9\end{array}$ & 54030.8 & $\begin{array}{c}\text { mrg1 (HRC-I) } \\
\text { mrg1 (EPIC) }\end{array}$ & $\begin{array}{l}380.9 \\
432.8\end{array}$ & $\begin{array}{l}<6.2 \\
<2.6\end{array}$ & \\
\hline $2006-11 b$ & $\begin{array}{l}0: 42: 44.05 \\
41: 15: 02.2\end{array}$ & 54058.0 & $\begin{array}{l}\operatorname{mrg} 1 \text { (HRC-I) } \\
\text { mrg1 (EPIC) }\end{array}$ & $\begin{array}{l}353.6 \\
405.6\end{array}$ & $\begin{array}{l}<0.4 \\
<7.3\end{array}$ & $\begin{array}{l}\text { later re-brightening } \\
\text { as M31N 2006-12d }\end{array}$ \\
\hline 2006-11a & $\begin{array}{l}0: 42: 56.81 \\
41: 06: 18.4\end{array}$ & 54063.8 & $\begin{array}{l}\operatorname{mrg} 1 \text { (HRC-I) } \\
\text { mrg1 (EPIC) }\end{array}$ & $\begin{array}{l}347.9 \\
399.8\end{array}$ & $\begin{array}{l}<2.8 \\
<0.1\end{array}$ & \\
\hline $2006-11 c$ & $\begin{array}{l}0: 41: 33.23 \\
41: 10: 12.3\end{array}$ & 54069.8 & mrg1 (HRC-I) & 341.9 & $<11.3$ & $\begin{array}{l}\text { far off-axis } \\
\text { not in EPIC field of view }\end{array}$ \\
\hline $2006-12 a$ & $\begin{array}{l}0: 42: 21.09 \\
41: 13: 45.3\end{array}$ & 54085.0 & $\begin{array}{l}\operatorname{mrg} 1 \text { (HRC-I) } \\
\text { mrg1 (EPIC) }\end{array}$ & $\begin{array}{l}326.6 \\
378.6\end{array}$ & $\begin{array}{l}<0.9 \\
<0.9\end{array}$ & \\
\hline $2006-12 b$ & $\begin{array}{l}0: 42: 11.14 \\
41: 07: 43.8\end{array}$ & 54092.8 & $\begin{array}{l}\operatorname{mrg} 1 \text { (HRC-I) } \\
\text { mrg1 (EPIC) }\end{array}$ & $\begin{array}{l}318.9 \\
370.8\end{array}$ & $\begin{array}{l}<6.4 \\
<0.1\end{array}$ & \\
\hline $2006-12 c$ & $\begin{array}{l}0: 42: 43.27 \\
41: 17: 48.1\end{array}$ & 54093.8 & $\begin{array}{l}\operatorname{mrg} 1 \text { (HRC-I) } \\
\text { mrg1 (EPIC) }\end{array}$ & $\begin{array}{l}317.9 \\
369.8\end{array}$ & $\begin{array}{l}<0.6 \\
<0.5\end{array}$ & \\
\hline 2007-01a & $\begin{array}{l}0: 42: 51.13 \\
41: 14: 33.1\end{array}$ & 54114.8 & $\begin{array}{l}\operatorname{mrg} 1 \text { (HRC-I) } \\
\text { mrg1 (EPIC) }\end{array}$ & $\begin{array}{l}296.9 \\
348.8\end{array}$ & $\begin{array}{l}<0.8 \\
<1.8\end{array}$ & \\
\hline $2007-02 c$ & $\begin{array}{l}0: 42: 39.96 \\
41: 17: 21.9\end{array}$ & 54140.8 & $\begin{array}{l}\operatorname{mrg} 1 \text { (HRC-I) } \\
\text { mrg1 (EPIC) }\end{array}$ & $\begin{array}{l}270.9 \\
322.8\end{array}$ & $\begin{array}{l}<0.2 \\
<1.4\end{array}$ & \\
\hline $2007-03 a$ & $\begin{array}{l}0: 42: 53.60 \\
41: 12: 09.8\end{array}$ & 54163.8 & $\begin{array}{l}\operatorname{mrg} 1 \text { (HRC-I) } \\
\text { mrg1 (EPIC) }\end{array}$ & $\begin{array}{l}247.9 \\
299.8\end{array}$ & $\begin{array}{l}<2.5 \\
<2.5\end{array}$ & \\
\hline $2007-05 a$ & $\begin{array}{l}0: 43: 02.61 \\
41: 14: 41.4\end{array}$ & 54238.0 & $\begin{array}{l}\operatorname{mrg} 1 \text { (HRC-I) } \\
\text { mrg1 (EPIC) }\end{array}$ & $\begin{array}{l}173.6 \\
225.6\end{array}$ & $\begin{array}{l}<0.5 \\
<0.8\end{array}$ & \\
\hline 2007-06a & $\begin{array}{l}0: 41: 58.40 \\
41: 14: 10.9\end{array}$ & 54265.0 & $\begin{array}{l}\operatorname{mrg} 1 \text { (HRC-I) } \\
\text { mrg1 (EPIC) }\end{array}$ & $\begin{array}{l}146.6 \\
198.6\end{array}$ & $\begin{array}{l}<0.8 \\
<0.1\end{array}$ & \\
\hline $2007-07 a$ & $\begin{array}{l}0: 43: 04.05 \\
41: 17: 08.3\end{array}$ & 54286.0 & $\begin{array}{l}\operatorname{mrg} 1 \text { (HRC-I) } \\
\text { mrg1 (EPIC) }\end{array}$ & $\begin{array}{l}125.6 \\
177.6\end{array}$ & $\begin{array}{l}<0.3 \\
<0.2\end{array}$ & \\
\hline $2007-07 b$ & $\begin{array}{l}0: 42: 45.89 \\
41: 18: 04.2\end{array}$ & 54289.0 & $\begin{array}{l}\operatorname{mrg} 1 \text { (HRC-I) } \\
\text { mrg1 (EPIC) }\end{array}$ & $\begin{array}{l}122.6 \\
174.6\end{array}$ & $\begin{array}{l}<0.5 \\
<0.4\end{array}$ & \\
\hline $2007-07 \mathrm{c}$ & $\begin{array}{l}0: 43: 03.29 \\
41: 14: 52.9\end{array}$ & 54300.0 & $\begin{array}{l}\operatorname{mrg} 1 \text { (HRC-I) } \\
\text { mrg1 (EPIC) }\end{array}$ & $\begin{array}{l}111.6 \\
163.6\end{array}$ & $\begin{array}{l}<0.3 \\
<1.2\end{array}$ & \\
\hline $2007-07 d$ & $\begin{array}{l}0: 42: 59.49 \\
41: 15: 06.5\end{array}$ & 54305.0 & $\begin{array}{l}\operatorname{mrg} 1 \text { (HRC-I) } \\
\text { mrg1 (EPIC) }\end{array}$ & $\begin{array}{l}106.6 \\
158.6\end{array}$ & $\begin{array}{l}<0.5 \\
<1.3\end{array}$ & \\
\hline $2007-07 e$ & $\begin{array}{l}0: 42: 43.29 \\
41: 17: 44.1\end{array}$ & 54306.5 & $\begin{array}{l}\operatorname{mrg} 1 \text { (HRC-I) } \\
\text { mrg1 (EPIC) }\end{array}$ & $\begin{array}{l}105.1 \\
157.1\end{array}$ & $\begin{array}{l}<1.0 \\
<0.9\end{array}$ & \\
\hline $2007-08 \mathrm{e}$ & $\begin{array}{l}0: 42: 44.70 \\
41: 16: 36.2\end{array}$ & 54333.0 & $\begin{array}{l}\operatorname{mrg} 1 \text { (HRC-I) } \\
\text { mrg1 (EPIC) }\end{array}$ & $\begin{array}{r}78.6 \\
130.6\end{array}$ & $\begin{array}{r}<0.8 \\
<28.8\end{array}$ & close to M 31 centre \\
\hline $2007-08 c$ & $\begin{array}{l}0: 42: 29.40 \\
41: 18: 24.8\end{array}$ & 54342.0 & $\begin{array}{l}\operatorname{mrg} 1 \text { (HRC-I) } \\
\text { mrg1 (EPIC) }\end{array}$ & $\begin{array}{r}69.6 \\
121.6\end{array}$ & $\begin{array}{l}<0.8 \\
<0.4\end{array}$ & \\
\hline 2007-10a & $\begin{array}{l}0: 42: 55.95 \\
41: 03: 22.0\end{array}$ & 54379.0 & mrg1 (HRC-I) & 32.6 & $<9.9$ & $\begin{array}{l}\text { far off-axis } \\
\text { not in EPIC field of view }\end{array}$ \\
\hline $2007-11 c$ & $\begin{array}{l}0: 43: 04.14 \\
41: 15: 54.3\end{array}$ & 54416.0 & $\begin{array}{r}8527 \text { (HRC-I) } \\
8528 \text { (HRC-I) } \\
8529 \text { (HRC-I) } \\
8530 \text { (HRC-I) } \\
\text { mrg1 (EPIC) }\end{array}$ & $\begin{array}{r}5.8 \\
16.8 \\
25.6 \\
35.5 \\
47.6\end{array}$ & $\begin{array}{l}<3.8 \\
<2.0 \\
<3.1 \\
<3.8 \\
<1.9\end{array}$ & \\
\hline 2008-01a & $\begin{array}{l}0: 42: 58.54 \\
41: 14: 44.1\end{array}$ & 54485.2 & $\begin{array}{l}0505720501 \text { (EPIC) } \\
0505720601 \text { (EPIC) }\end{array}$ & $\begin{array}{r}7.7 \\
18.0\end{array}$ & $\begin{array}{l}<2.2 \\
<1.4\end{array}$ & \\
\hline
\end{tabular}

Notes. As for Table 2 . 
M. Henze et al.: X-ray monitoring of classical novae in M 31 in 2007/8 and 2008/9. II.

Table 7. Upper limits for M $31 \mathrm{CNe}$ with outburst from about one year before the start of the 2008/9 monitoring till its end.

\begin{tabular}{|c|c|c|c|c|c|c|}
\hline \multicolumn{3}{|c|}{ Optical nova candidate } & \multicolumn{3}{|l|}{ X-ray measurements } & \multirow[b]{2}{*}{ Comment } \\
\hline $\begin{array}{l}\text { Name } \\
\text { M31N }\end{array}$ & $\begin{array}{l}\text { RA }(\mathrm{h}: \mathrm{m}: \mathrm{s})^{a} \\
\operatorname{Dec}(\mathrm{d}: \mathrm{m}: \mathrm{s})^{a}\end{array}$ & $\begin{array}{l}\mathrm{MJD}^{b} \\
(\mathrm{~d})\end{array}$ & $\begin{array}{l}\text { Observation }^{d} \\
\text { ID }\end{array}$ & $\begin{array}{l}\Delta t^{e} \\
(\mathrm{~d})\end{array}$ & $\begin{array}{c}L_{50}{ }^{f} \\
\left(10^{36} \mathrm{erg} \mathrm{s}^{-1}\right)\end{array}$ & \\
\hline $2007-10 a$ & $\begin{array}{l}0: 42: 55.95 \\
41: 03: 22.0\end{array}$ & 54379.0 & $\operatorname{mrg} 2($ HRC-I $)$ & 399.3 & $<17.0$ & $\begin{array}{l}\text { far off-axis } \\
\text { not in EPIC field of view }\end{array}$ \\
\hline $2007-11 \mathrm{c}$ & $\begin{array}{l}0: 43: 04.14 \\
41: 15: 54.3\end{array}$ & 54416.0 & $\begin{array}{r}\operatorname{mrg} 2 \text { (HRC-I) } \\
\text { mrg2 (EPIC) }\end{array}$ & $\begin{array}{l}362.3 \\
414.1\end{array}$ & $\begin{array}{l}<0.5 \\
<2.3\end{array}$ & \\
\hline 2008-01a & $\begin{array}{l}0: 42: 58.54 \\
41: 14: 44.1\end{array}$ & 54485.2 & $\begin{array}{r}\operatorname{mrg} 2(\text { HRC-I) } \\
\operatorname{mrg} 2 \text { (EPIC) }\end{array}$ & $\begin{array}{l}293.1 \\
344.9\end{array}$ & $\begin{array}{l}<0.6 \\
<0.6\end{array}$ & \\
\hline $2008-02 a$ & $\begin{array}{l}0: 42: 30.38 \\
41: 09: 53.8\end{array}$ & 54503.2 & $\begin{array}{r}\operatorname{mrg} 2 \text { (HRC-I) } \\
\operatorname{mrg} 2 \text { (EPIC) }\end{array}$ & $\begin{array}{l}275.1 \\
326.9\end{array}$ & $\begin{array}{l}<0.9 \\
<1.0\end{array}$ & \\
\hline $2008-03 b$ & $\begin{array}{l}0: 42: 34.21 \\
41: 16: 44.4\end{array}$ & 54527.8 & $\begin{array}{r}\operatorname{mrg} 2 \text { (HRC-I) } \\
\operatorname{mrg} 2(\text { EPIC) }\end{array}$ & $\begin{array}{l}250.6 \\
302.4\end{array}$ & $\begin{array}{l}<0.6 \\
<1.3\end{array}$ & \\
\hline $2008-05 c$ & $\begin{array}{l}0: 43: 12.08 \\
41: 19: 15.8\end{array}$ & 54612.5 & $\begin{array}{r}\operatorname{mrg} 2(\text { HRC-I) } \\
\operatorname{mrg} 2 \text { (EPIC) }\end{array}$ & $\begin{array}{l}165.8 \\
217.6\end{array}$ & $\begin{array}{l}<2.2 \\
<0.4\end{array}$ & \\
\hline $2008-07 a$ & $\begin{array}{l}0: 42: 34.42 \\
41: 18: 15.7\end{array}$ & 54619.0 & $\begin{array}{r}\operatorname{mrg} 2 \text { (HRC-I) } \\
\operatorname{mrg} 2 \text { (EPIC) }\end{array}$ & $\begin{array}{l}159.3 \\
211.1\end{array}$ & $\begin{array}{l}<0.6 \\
<0.1\end{array}$ & \\
\hline $2008-06 b$ & $\begin{array}{l}0: 42: 27.81 \\
41: 14: 48.2\end{array}$ & 54643.0 & $\begin{array}{r}\operatorname{mrg} 2(\text { HRC-I) } \\
\operatorname{mrg} 2 \text { (EPIC) }\end{array}$ & $\begin{array}{l}135.3 \\
187.1\end{array}$ & $\begin{array}{l}<0.8 \\
<0.4\end{array}$ & \\
\hline $2008-06 c$ & $\begin{array}{l}0: 43: 08.30 \\
41: 18: 38.0\end{array}$ & 54645.0 & $\begin{array}{r}\operatorname{mrg} 2 \text { (HRC-I) } \\
\text { mrg2 (EPIC) }\end{array}$ & $\begin{array}{l}133.3 \\
185.1\end{array}$ & $\begin{array}{l}<1.7 \\
<0.3\end{array}$ & \\
\hline $2008-07 b$ & $\begin{array}{l}0: 43: 27.28 \\
41: 10: 03.3\end{array}$ & 54669.2 & $\begin{array}{r}\operatorname{mrg} 2(\text { HRC-I) } \\
\operatorname{mrg} 2(\text { EPIC) }\end{array}$ & $\begin{array}{l}109.1 \\
160.9\end{array}$ & $\begin{array}{l}<4.0 \\
<0.6\end{array}$ & \\
\hline 2008-08a & $\begin{array}{l}0: 42: 44.99 \\
41: 17: 07.7\end{array}$ & 54688.0 & $\begin{array}{r}\operatorname{mrg} 2(\text { HRC-I) } \\
\operatorname{mrg} 2(\text { EPIC) }\end{array}$ & $\begin{array}{r}90.3 \\
142.1\end{array}$ & $\begin{array}{l}<1.7 \\
<3.0\end{array}$ & \\
\hline $2008-08 b$ & $\begin{array}{l}0: 42: 52.38 \\
41: 16: 12.9\end{array}$ & 54688.0 & $\begin{array}{r}\operatorname{mrg} 2 \text { (HRC-I) } \\
\operatorname{mrg} 2 \text { (EPIC) }\end{array}$ & $\begin{array}{r}90.3 \\
142.1\end{array}$ & $\begin{array}{l}<1.5 \\
<5.4\end{array}$ & \\
\hline $2008-08 c$ & $\begin{array}{l}0: 42: 40.51 \\
41: 26: 18.0\end{array}$ & 54706.2 & $\begin{array}{r}\operatorname{mrg} 2 \text { (HRC-I) } \\
\text { mrg2 (EPIC) }\end{array}$ & $\begin{array}{r}72.1 \\
123.9\end{array}$ & $\begin{array}{l}<1.8 \\
<0.3\end{array}$ & \\
\hline 2008-09a & $\begin{array}{l}0: 41: 46.72 \\
41: 07: 52.1\end{array}$ & 54722.2 & $\begin{array}{r}\operatorname{mrg} 2(\text { HRC-I) } \\
\operatorname{mrg} 2 \text { (EPIC) }\end{array}$ & $\begin{array}{r}56.1 \\
107.9\end{array}$ & $\begin{array}{r}<13.5 \\
<0.9\end{array}$ & far off-axis \\
\hline $2008-09 c$ & $\begin{array}{l}0: 42: 51.42 \\
41: 01: 54.0\end{array}$ & 54724.2 & mrg2 (HRC-I) & 54.1 & $<18.1$ & $\begin{array}{l}\text { far off-axis } \\
\text { not in EPIC field of view }\end{array}$ \\
\hline $2008-10 b$ & $\begin{array}{l}0: 43: 02.42 \\
41: 14: 09.9\end{array}$ & 54745.0 & $\begin{array}{r}\operatorname{mrg} 2 \text { (HRC-I) } \\
\text { mrg2 (EPIC) }\end{array}$ & $\begin{array}{l}33.3 \\
85.1\end{array}$ & $\begin{array}{l}<2.5 \\
<0.6\end{array}$ & \\
\hline $2008-10 \mathrm{c}$ & $\begin{array}{l}0: 42: 48.50 \\
41: 13: 49.8\end{array}$ & 54759.0 & $\begin{array}{r}\operatorname{mrg} 2 \text { (HRC-I) } \\
\operatorname{mrg} 2 \text { (EPIC) }\end{array}$ & $\begin{array}{l}19.3 \\
71.1\end{array}$ & $\begin{array}{l}<0.8 \\
<0.1\end{array}$ & \\
\hline 2008-11d & $\begin{array}{l}0: 42: 57.30 \\
41: 15: 41.1\end{array}$ & 54795.0 & $\begin{array}{r}9827 \text { (HRC-I) } \\
9828 \text { (HRC-I) } \\
9829 \text { (HRC-I) } \\
10838 \text { (HRC-I) } \\
\text { mrg2 (EPIC) } \\
10683 \text { (HRC-I) } \\
10684 \text { (HRC-I) }\end{array}$ & $\begin{array}{r}3.2 \\
12.4 \\
23.0 \\
23.5 \\
35.1 \\
83.9 \\
93.2\end{array}$ & $\begin{array}{l}<1.1 \\
<1.2 \\
<1.2 \\
<1.3 \\
<1.1 \\
<2.7 \\
<2.1\end{array}$ & \\
\hline $2008-12 b$ & $\begin{array}{l}0: 43: 04.85 \\
41: 17: 51.6\end{array}$ & 54829.8 & $\begin{array}{r}0551690201 \text { (EPIC) } \\
0551690301 \text { (EPIC) } \\
0551690401 \text { (EPIC) } \\
0551690501 \text { (EPIC) } \\
0551690601 \text { (EPIC) } \\
10683 \text { (HRC-I) } \\
10684 \text { (HRC-I) }\end{array}$ & $\begin{array}{r}0.4 \\
10.5 \\
17.1 \\
28.6 \\
36.8 \\
49.1 \\
58.4\end{array}$ & $\begin{array}{l}<1.9 \\
<3.0 \\
<4.8 \\
<6.3 \\
<3.4 \\
<0.6 \\
<0.6\end{array}$ & \\
\hline $2009-02 b$ & $\begin{array}{l}0: 42: 27.77 \\
41: 13: 42.4 \\
\end{array}$ & 54882.2 & 10684 (HRC-I) & 5.9 & $<1.9$ & \\
\hline
\end{tabular}

Notes. As for Table 2. 
Table 8. Non-nova SSSs detected during the monitoring.

\begin{tabular}{|c|c|c|c|c|c|}
\hline $\begin{array}{l}\text { Name }^{a} \\
\text { XMMM31 }\end{array}$ & $\begin{array}{l}\text { RA }(\mathrm{h}: \mathrm{m}: \mathrm{s})^{b} \\
\operatorname{Dec}(\mathrm{d}: \mathrm{m}: \mathrm{s})^{a}\end{array}$ & $\begin{array}{l}\text { Observation } \\
\text { ID }\end{array}$ & $\begin{array}{l}\mathrm{MJD}^{c} \\
\text { (d) }\end{array}$ & $\begin{array}{c}L_{50}{ }^{d} \\
\left(10^{36} \mathrm{erg} \mathrm{s}^{-1}\right)\end{array}$ & Comment $^{e}$ \\
\hline J004252.5+411541 & $\begin{array}{l}0: 42: 52.50 \\
41: 15: 40.1\end{array}$ & $\begin{array}{r}8526 \text { (HRC-I) } \\
8527 \text { (HRC-I) } \\
8528 \text { (HRC-I) } \\
8529 \text { (HRC-I) } \\
8530 \text { (HRC-I) } \\
0505720201 \text { (EPIC) } \\
0505720301 \text { (EPIC) } \\
0505720401 \text { (EPIC) } \\
0505720501 \text { (EPIC) } \\
0505720601 \text { (EPIC) } \\
9825 \text { (HRC-I) } \\
9826 \text { (HRC-I) } \\
9827 \text { (HRC-I) } \\
9828 \text { (HRC-I) } \\
9829 \text { (HRC-I) } \\
10838 \text { (HRC-I) } \\
0551690201 \text { (EPIC) } \\
0551690301 \text { (EPIC) } \\
0551690401 \text { (EPIC) } \\
0551690501 \text { (EPIC) } \\
0551690601 \text { (EPIC) } \\
10683 \text { (HRC-I) } \\
10684 \text { (HRC-I) }\end{array}$ & $\begin{array}{l}54411.6 \\
54421.8 \\
54432.8 \\
54441.6 \\
54451.5 \\
54463.6 \\
54473.3 \\
54483.6 \\
54492.9 \\
54503.2 \\
54778.3 \\
54787.1 \\
54798.2 \\
54807.4 \\
54818.0 \\
54818.5 \\
54830.1 \\
54840.3 \\
54846.9 \\
54858.3 \\
54866.6 \\
54878.9 \\
54888.2\end{array}$ & $\begin{array}{r}267.1 \pm 9.6 \\
294.4 \pm 10.2 \\
361.7 \pm 14.1 \\
217.8 \pm 11.3 \\
248.7 \pm 11.6 \\
203.1 \pm 2.3 \\
228.1 \pm 2.5 \\
178.1 \pm 2.6 \\
215.7 \pm 3.5 \\
247.8 \pm 3.1 \\
156.1 \pm 7.3 \\
232.3 \pm 9.0 \\
362.2 \pm 14.2 \\
301.6 \pm 12.8 \\
261.5 \pm 16.7 \\
303.5 \pm 18.0 \\
244.5 \pm 3.0 \\
189.7 \pm 2.6 \\
224.2 \pm 4.6 \\
230.6 \pm 3.1 \\
176.0 \pm 3.2 \\
255.7 \pm 10.8 \\
224.2 \pm 10.0\end{array}$ & $217.7 \mathrm{~s}$ period (1) \\
\hline J004318.8+412017 & $\begin{array}{l}0: 43: 18.80 \\
41: 20: 16.1\end{array}$ & $\begin{array}{r}8526 \text { (HRC-I) } \\
8527 \text { (HRC-I) } \\
8528 \text { (HRC-I) } \\
8529 \text { (HRC-I) } \\
8530 \text { (HRC-I) } \\
0505720201 \text { (EPIC) } \\
0505720301 \text { (EPIC) } \\
0505720401 \text { (EPIC) } \\
0505720501 \text { (EPIC) } \\
0505720601 \text { (EPIC) } \\
9825 \text { (HRC-I) } \\
9826 \text { (HRC-I) } \\
9827 \text { (HRC-I) } \\
9828 \text { (HRC-I) } \\
9829 \text { (HRC-I) } \\
10838 \text { (HRC-I) } \\
0551690201 \text { (EPIC) } \\
0551690301 \text { (EPIC) } \\
0551690401 \text { (EPIC) } \\
0551690501 \text { (EPIC) } \\
0551690601 \text { (EPIC) } \\
10683 \text { (HRC-I) } \\
10684 \text { (HRC-I) }\end{array}$ & $\begin{array}{l}54411.6 \\
54421.8 \\
54432.8 \\
54441.6 \\
54451.5 \\
54463.6 \\
54473.3 \\
54483.6 \\
54492.9 \\
54503.2 \\
54778.3 \\
54787.1 \\
54798.2 \\
54807.4 \\
54818.0 \\
54818.5 \\
54830.1 \\
54840.3 \\
54846.9 \\
54858.3 \\
54866.6 \\
54878.9 \\
54888.2\end{array}$ & $\begin{aligned} &<5.9 \\
&<11.0 \\
&<1.6 \\
&<1.5 \\
& 10.2 \pm 2.6 \\
& 9.4 \pm 0.9 \\
& 4.6 \pm 0.6 \\
&<3.8 \\
& 5.3 \pm 0.9 \\
& 1.1 \pm 0.4 \\
& 20.5 \pm \pm 5.4 \\
& 14.5 \pm 4.2 \\
&<2.7 \\
&<1.6 \\
&<7.2 \\
&<10.8 \\
& 3.0 \pm 0.6 \\
& 2.5 \pm 0.7 \\
&<3.3 \\
&<1.9 \\
&<2.4 \\
&<7.7 \\
&<3.5\end{aligned}$ & foreground polar(?) (2) \\
\hline
\end{tabular}


M. Henze et al.: X-ray monitoring of classical novae in M 31 in 2007/8 and 2008/9. II.

Table 8. continued.

\begin{tabular}{|c|c|c|c|c|c|}
\hline $\begin{array}{l}\text { Name }^{a} \\
\text { XMMM31 }\end{array}$ & $\begin{array}{l}\text { RA }(\mathrm{h}: \mathrm{m}: \mathrm{s})^{b} \\
\operatorname{Dec}(\mathrm{d}: \mathrm{m}: \mathrm{s})^{a}\end{array}$ & $\begin{array}{l}\text { Observation } \\
\text { ID }\end{array}$ & $\begin{array}{c}\mathrm{MJD}^{c} \\
\text { (d) }\end{array}$ & $\begin{array}{c}L_{50}{ }^{d} \\
\left(10^{36} \mathrm{erg} \mathrm{s}^{-1}\right)\end{array}$ & Comment $^{e}$ \\
\hline J004318.7+411804 & $\begin{array}{l}0: 43: 18.70 \\
41: 18: 05.2\end{array}$ & $\begin{array}{r}8526 \text { (HRC-I) } \\
8527 \text { (HRC-I) } \\
8528 \text { (HRC-I) } \\
8529 \text { (HRC-I) } \\
8530 \text { (HRC-I) } \\
0505720201 \text { (EPIC) } \\
0505720301 \text { (EPIC) } \\
0505720401 \text { (EPIC) } \\
0505720501 \text { (EPIC) } \\
0505720601 \text { (EPIC) } \\
9825 \text { (HRC-I) } \\
9826 \text { (HRC-I) } \\
9827 \text { (HRC-I) } \\
9828 \text { (HRC-I) } \\
9829 \text { (HRC-I) } \\
10838 \text { (HRC-I) } \\
0551690201 \text { (EPIC) } \\
0551690301 \text { (EPIC) } \\
0551690401 \text { (EPIC) } \\
0551690501 \text { (EPIC) } \\
0551690601 \text { (EPIC) } \\
10683 \text { (HRC-I) } \\
10684 \text { (HRC-I) }\end{array}$ & $\begin{array}{l}54411.6 \\
54421.8 \\
54432.8 \\
54441.6 \\
54451.5 \\
54463.6 \\
54473.3 \\
54483.6 \\
54492.9 \\
54503.2 \\
54778.3 \\
54787.1 \\
54798.2 \\
54807.4 \\
54818.0 \\
54818.5 \\
54830.1 \\
54840.3 \\
54846.9 \\
54858.3 \\
54866.6 \\
54878.9 \\
54888.2\end{array}$ & $\begin{aligned} & <6.5 \\
& <3.3 \\
& <9.0 \\
& <8.6 \\
& <6.1 \\
1.1 & \pm 0.4 \\
0.9 & \pm 0.3 \\
0.6 \pm 0.3 & \\
& <6.9 \\
1.7 & \pm 0.5 \\
9.7 & \pm 3.3 \\
& <8.1 \\
& <4.3 \\
& <4.3 \\
& <10.1 \\
9.5 & \pm 3.3 \\
2.4 & \pm 0.6 \\
1.9 & \pm 0.5 \\
& <3.2 \\
4.8 & \pm 1.9 \\
& <2.1 \\
& <7.2 \\
& <2.8\end{aligned}$ & strongly variable (2) \\
\hline
\end{tabular}

Notes. ${ }^{(a)}$ Source name from the catalogue of Stiele et al. (2008); ${ }^{(b)}$ RA, Dec are given in J2000.0; ${ }^{(c)}$ modified Julian Date MJD = JD - 2400000.5 ; (d) unabsorbed equivalent luminosity in $0.2-10.0 \mathrm{keV}$ band assuming a $50 \mathrm{eV}$ blackbody spectrum with Galactic foreground absorption, luminosity errors are $1 \sigma$, upper limits are $3 \sigma ;^{(e)}$ (1) Trudolyubov \& Priedhorsky (2008), (2) Williams et al. (2006), (3) Orio (2006). 


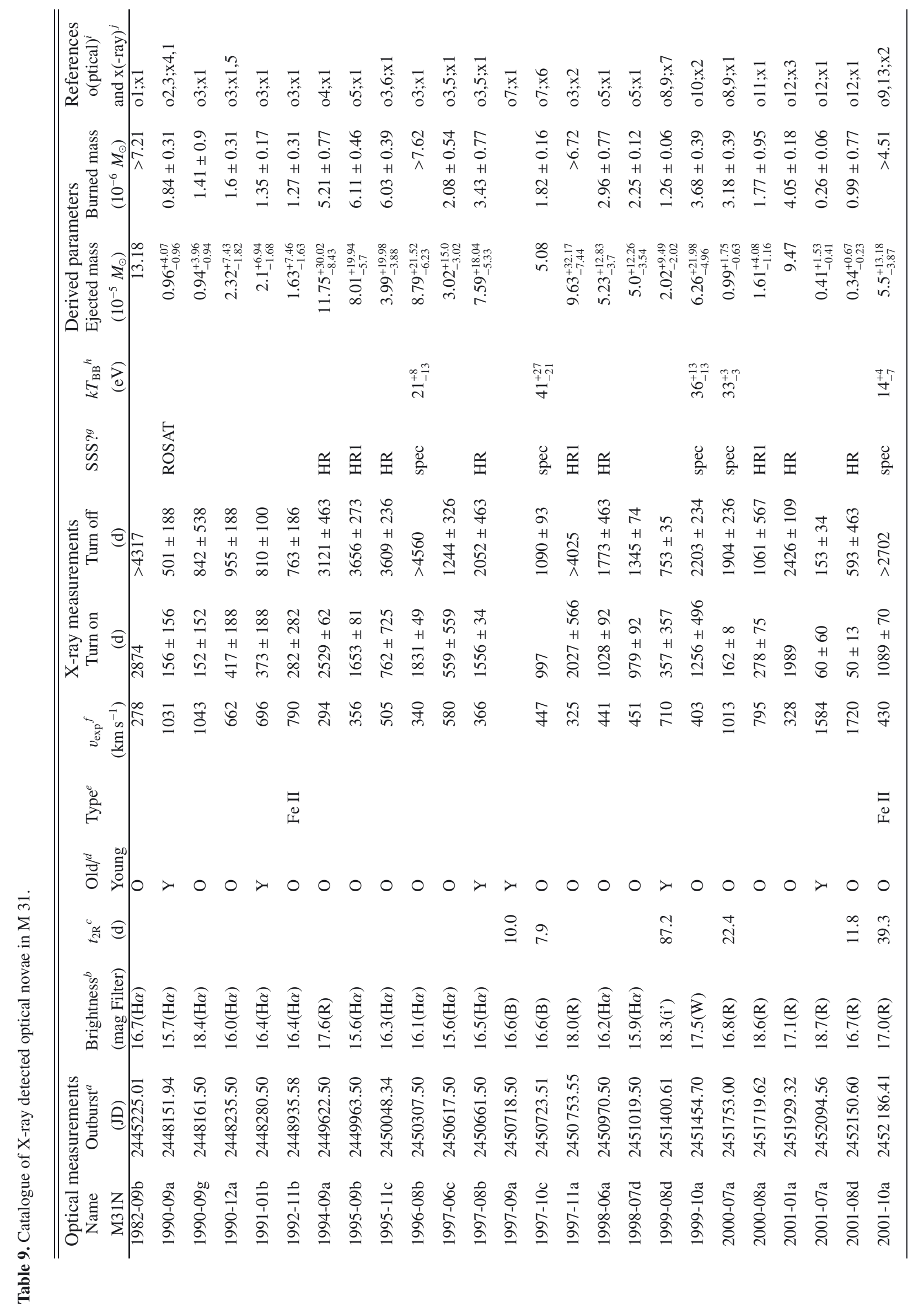


M. Henze et al.: X-ray monitoring of classical novae in M 31 in 2007/8 and 2008/9. II.

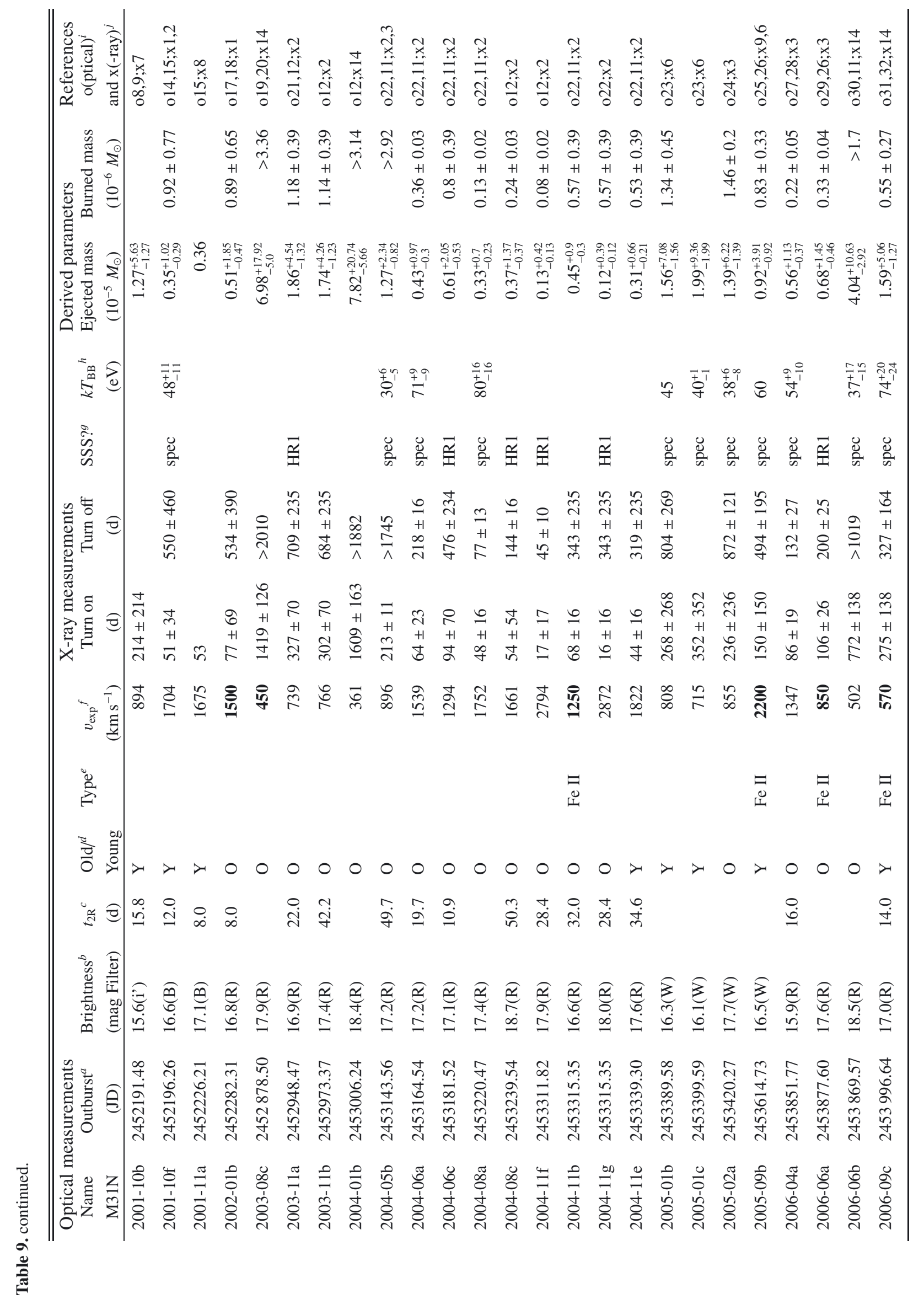




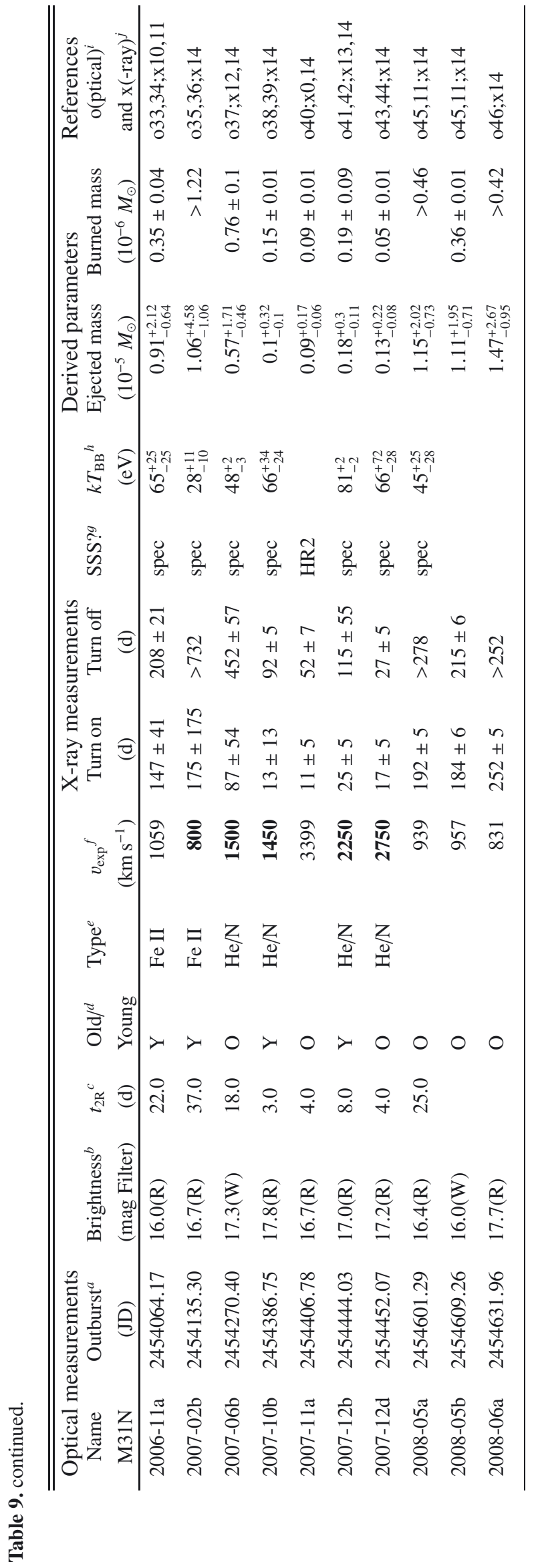

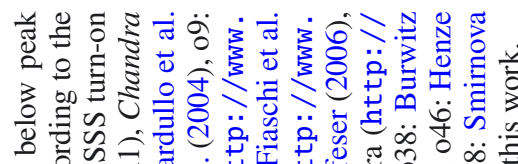
on

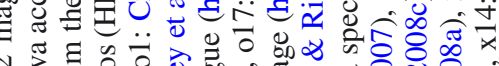

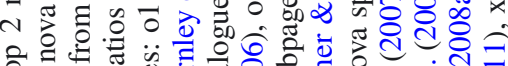
O० .

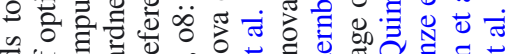

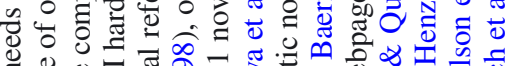

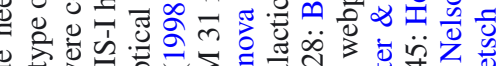
ष

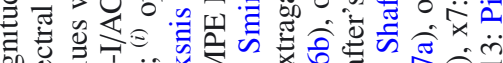

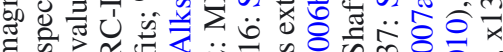

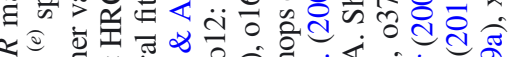

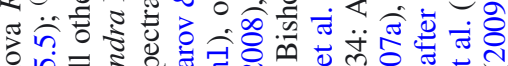

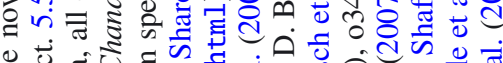

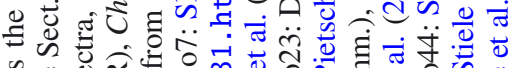

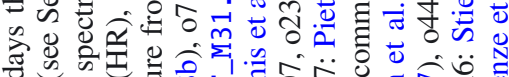
ઘ

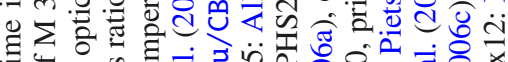
毛出

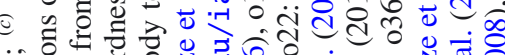

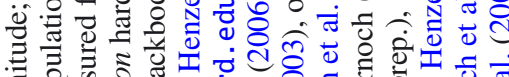

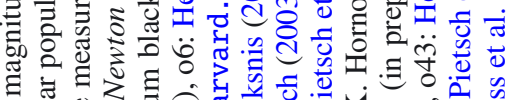

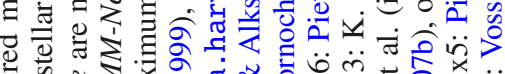
क्षे

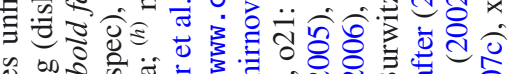

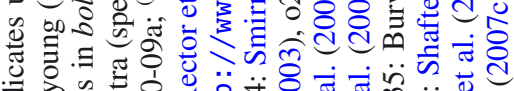

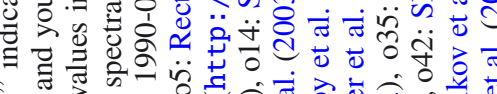

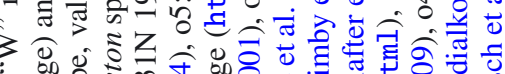

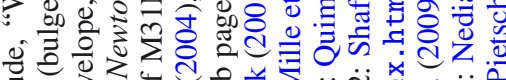

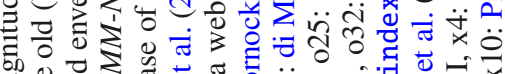

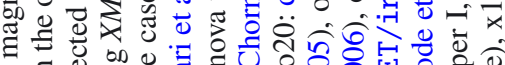

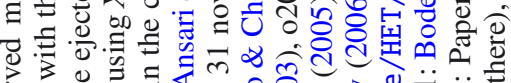

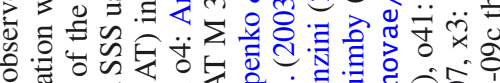

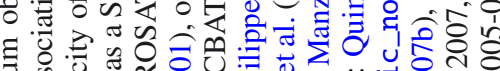

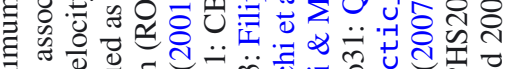

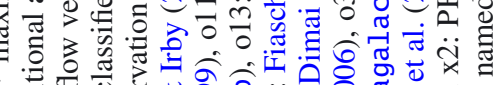

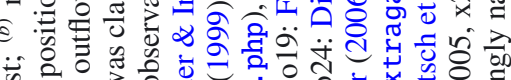

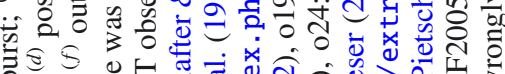

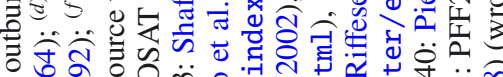

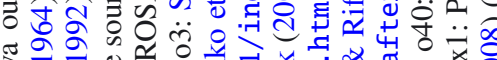

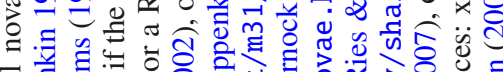

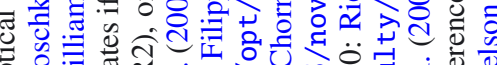

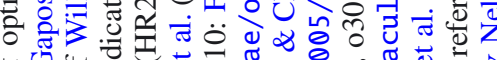
t)

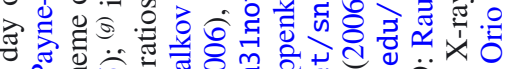

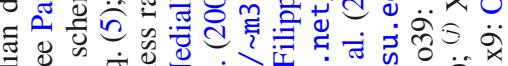

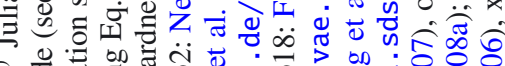

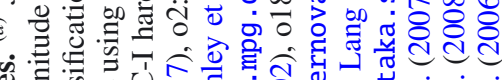

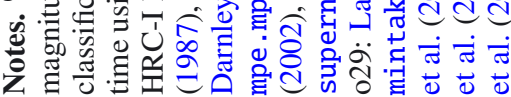

